WHC-SP-0098-5

UC. 600

\title{
Routine Operational Environmental Monitoring Schedule, CY 1994
}

J. W. Schmidt

Date Published

December 1993

Prepared for the U.S. Department of Energy Office of Environmental Restoration and Waste Management

Q2 Westinghouse

P.O. Box 1970

Hanford Company Richland, Washington 99352

Hanford Operations and Engineering Contractor for the

U.S. Department of Energy under Contract DE-AC06-87RL10930 


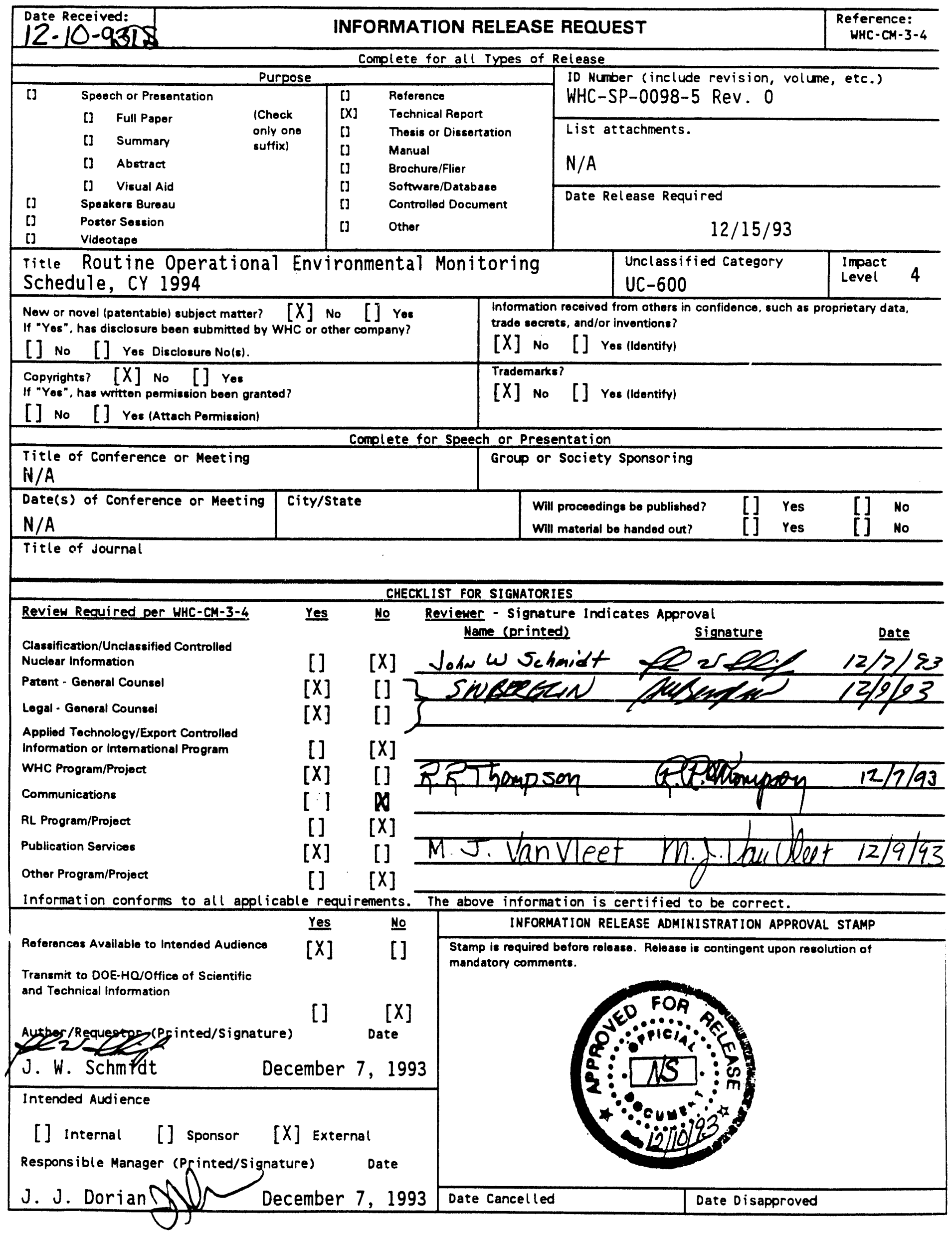


WHC-SP-0098-5

Rev. 0

Issue Date: 12/15/93

Document Title: Routine Operational Environmental Monitoring Schedule, CY 1994

Prepared by:

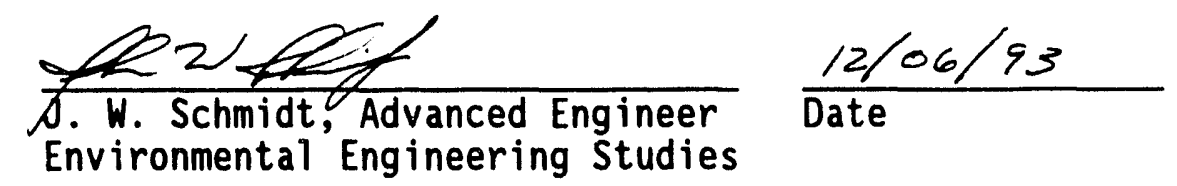

Reviewed

Approved by:

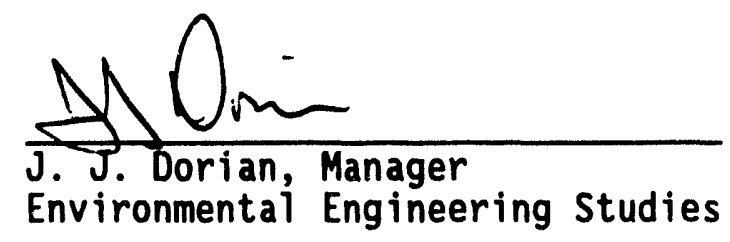

$\frac{12 / 06 / 93}{\text { Date }}$

Reviewed

Approved by:

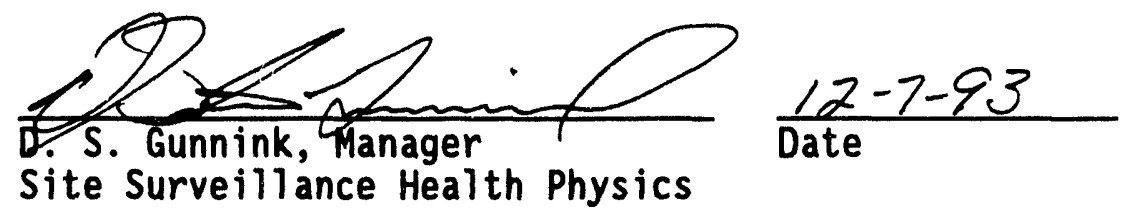

Reviewed

Approved by:
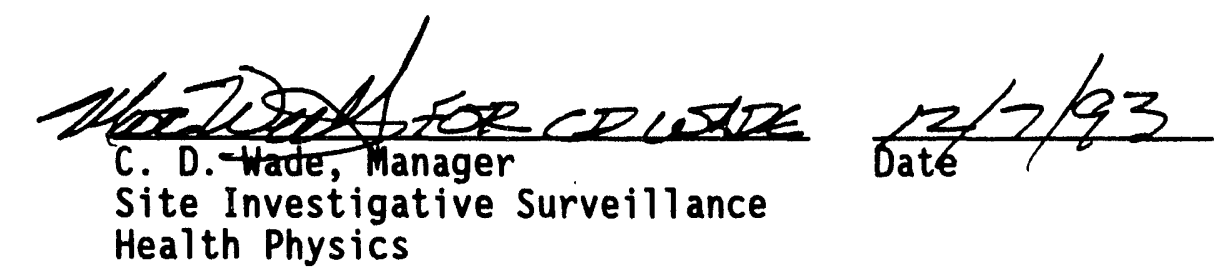

Health Physics 
WHC-SP-0098-5

Rev. 0

Issue Date: 12/15/93

\section{CONTENTS}

\section{SCHEDULE ROUTINES}

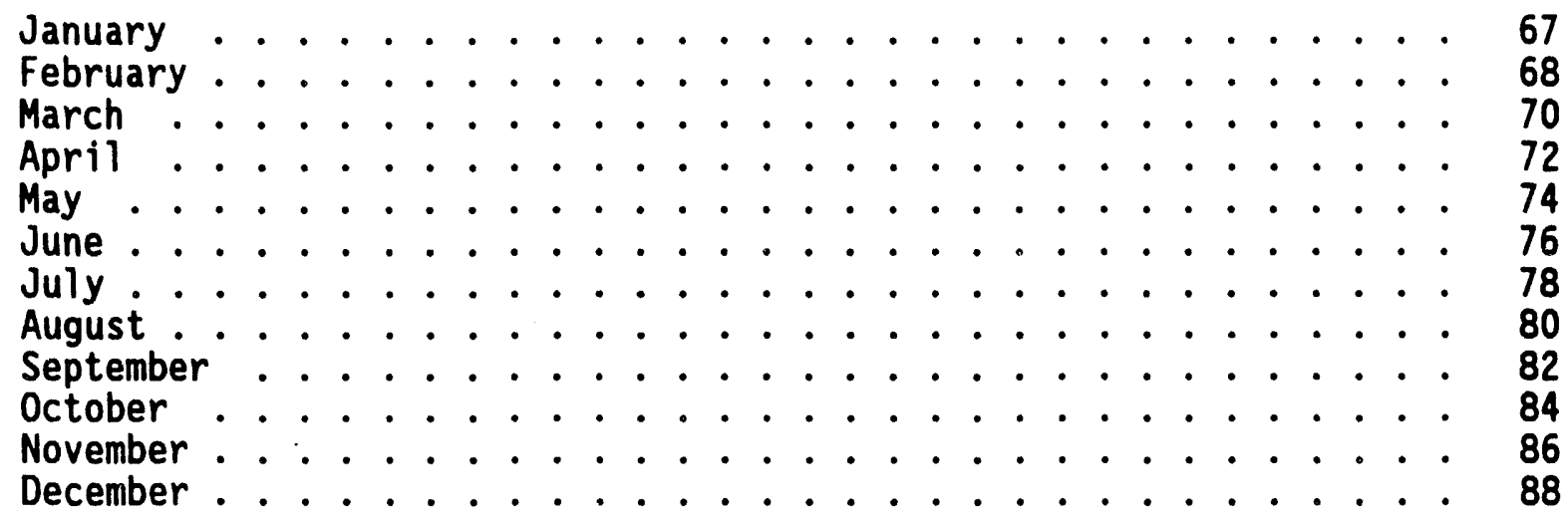




\section{LIST OF FIGURES}

Hanford Site ........................ . 3

100-B and 100-C Areas Waste Sites................ 4

100-K Area Waste Sites .................... 5

100-N Area Waste Sites ................... . . 6

100-D Area Waste Sites .................. . 7

100-H Area Waste Sites . . . . . . . . . . . . . . . 8

100-F Area Waste Sites ................... . 9

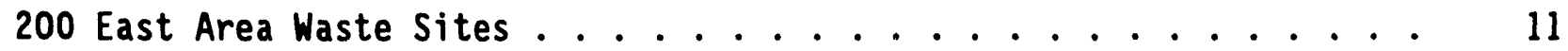

200 East Area Waste Sites, N.E. Quadrant . . . . . . . . . . . 13/14

200 East Area Waste Sites, N.W. Quadrant . . . . . . . . . . 15/16

200 East Area Waste Sites, S.E. Quadrant . . . . . . . . . . . 17/18

200 East Area Waste Sites, S.W. Quadrant . . . . . . . . . . . 19/20

200 West Area Waste Sites . . . . . . . . . . . . . . . . 21/22

200 West Area Waste Sites, N.E. Quadrant .............. . 23/24

200 West Area Waste Sites, N.W. Quadrant . . . . . . . . . . 25/26

200 West Area Waste Sites, S.E. Quadrant . . . . . . . . . . 27/28

200 West Area Waste Sites, S.W. Quadrant . . . . . . . . . . . 29/30

200 East Area Waste Management Facility Pipelines . . . . . . . . . 31/32

200 West Area Waste Management Facility Pipelines . . . . . . . . . 33/34

300 Area Waste Sites .................... 35

400 Area Waste Sites .................... 36

600 Area Waste Sites .. . . . . . . . . . . . . . . . 37

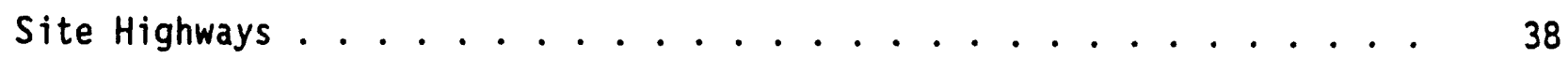

200 East Area Roads and Railways ... . . . . . . . . . . . 39

200 West Area Roads and Railways .................. 40 
LIST OF FIGURES (cont)

Site Railways...................... 41

100-N Area Ambient Air Sample Location . . . . . . . . . . . . . 42

100-K Area Ambient Air Sample Location ... . . . . . . . . 43

200 East Area Air Sample Locations . . . . . . . . . . . . . . 44

200 West Area Air Sample Locations . . . . . . . . . . . . . 45

200 East/West Areas Vent Station Air Sample Locations . . . . . . . . 46

600 Area Air Sample Location . . . . . . . . . . . . . . 47

100-N Area TLD Locations . . . . . . . . . . . . . . . 48

N Springs Dose Rate Locations . . . . . . . . . . . . . . 49

1301-N Building Dose Rate Locations . . . . . . . . . . . . . 50

1325-N Building Dose Rate Locations . . . . . . . . . . . . . . 51

100-K Area TLD Locations . . . . . . . . . . . . . . 52

200 East Area TLD Locations . . . . . . . . . . . . . 53

200 East Area, "A" Tank Farm TLD Locations . . . . . . . . . . 54

200 West Area TLD Locations . . . . . . . . . . . . . . 55

300 Area TLD Locations . . . . . . . . . . . . . . . 56

400 Area TLD Locations . . . . . . . . . . . . . . . 57

100-N Area Soil and Vegetation Sample Locations . . . . . . . . . . 58

200 East Area Soil and Vegetation Sample Locations . . . . . . . . 59

200 West Area Soil and Vegetation Sample Locations . . . . . . . . 60

600 Area Soil and Vegetation Sample Locations............ . 61

300 Area Soil and Vegetation Sample Locations............. 62

400 Area Soil and Vegetation Sample Locations ............ 63 
WHC-SP-0098-5

Rev. 0

Issue Date: $12 / 15 / 93$

\section{LIST OF FIGURES (cont)}

200 East Area Water, Sediment, and Aquatic Vegetation

Sample Locations . . . . . . . . . . . . . . . . . . . 64

200 West Area Water, Sediment, and Aquatic Vegetation

Sample Locations . . . . . . . . . . . . . . . . . . 65

N Springs Seepage Sample Locations . . . . . . . . . . . . . . 66 
WHC-SP-0098-5

Rev. 0

Issue Date: $12 / 15 / 93$

\section{PURPOSE}

This document provides Health Physics (HP) a schedule in accordance with the Environmental Compliance Manual, WHC-CM-7-5, of monitoring and sampling routines for the Operational Environmental Monitoring (OEM) Program during calendar year (CY) 1994. Every attempt will be made to consistently follow this schedule, any deviation from this schedule will be documented by a "DON'T SAY IT -- Write It!" (DSI), explaining the reason for the deviation issued by the scheduled performing organization to Environmental Engineering Studies (EES).

The survey frequencies for particular sites are determined by the technical judgment of EES and may depend on the site history, radiological status, use, and general conditions. Additional surveys may be requested at irregular frequencies if conditions warrant. All radioactive waste sites are scheduled to be surveyed annually at a minimum. Any newly discovered waste sites not documented by this schedule will be included in the revised schedule for CY 1995.

This schedule does not discuss the manpower needs nor does it list the monitoring equipment to be used in completing specific routines. Personnel performing routines for this schedule shall communicate any need for assistance in completing these routines to HP management and EES.

After the completion of each routine survey, a copy of the survey, maps, and data sheets will be forwarded to EES. These routine surveys will not be considered complete until this documentation is received. At the end of each month, HP shall forward a copy of the Routine Sign-off Sheet and a DSI validating the completion of the scheduled routines for that month. 
WHC-SP-0098-5

Rev. 0

Issue Date: $12 / 15 / 93$

This page intentionally left blank. 
WHC-SP-0098-5

Rev. 0

Is sue Date: 12/15/93

Hanford Site.

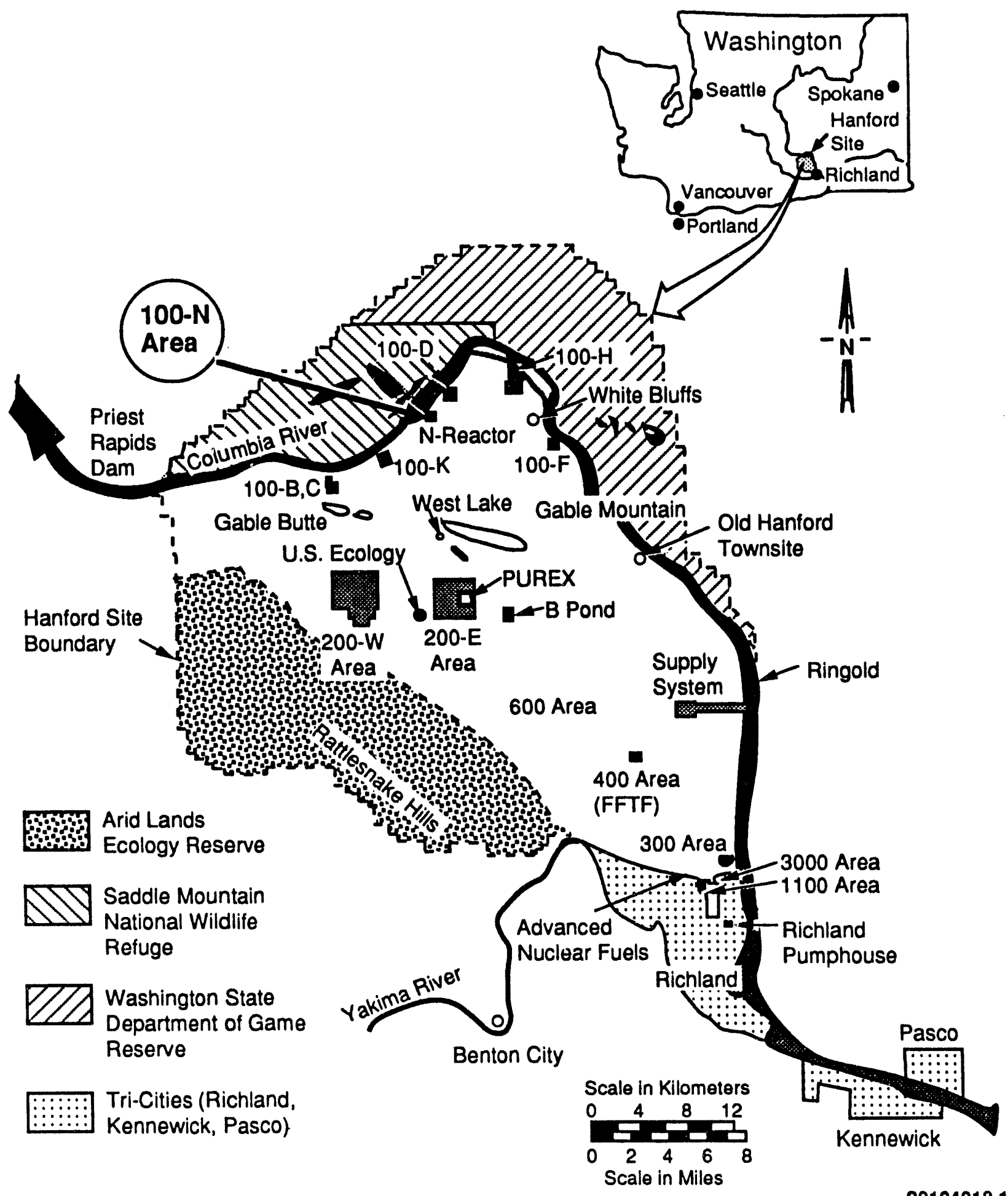

29104018.1 
WHC-SP-0098-5

Rev. 0

Issue Date: 12/15/93

100-B and 100-C Areas Waste Sites.

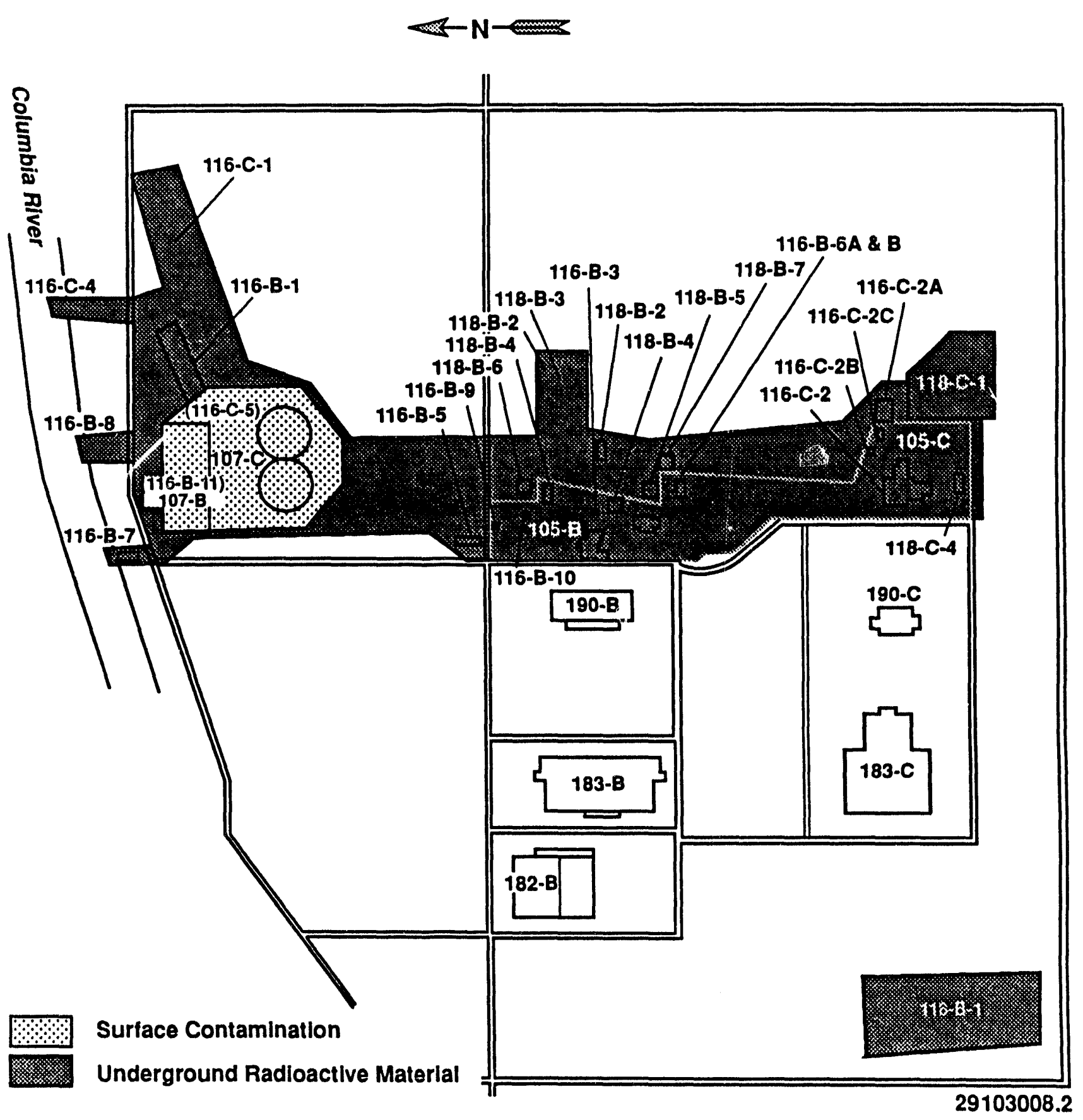


WHC-SP-0098-5

Rev. 0

Issue Date: 12/15/93

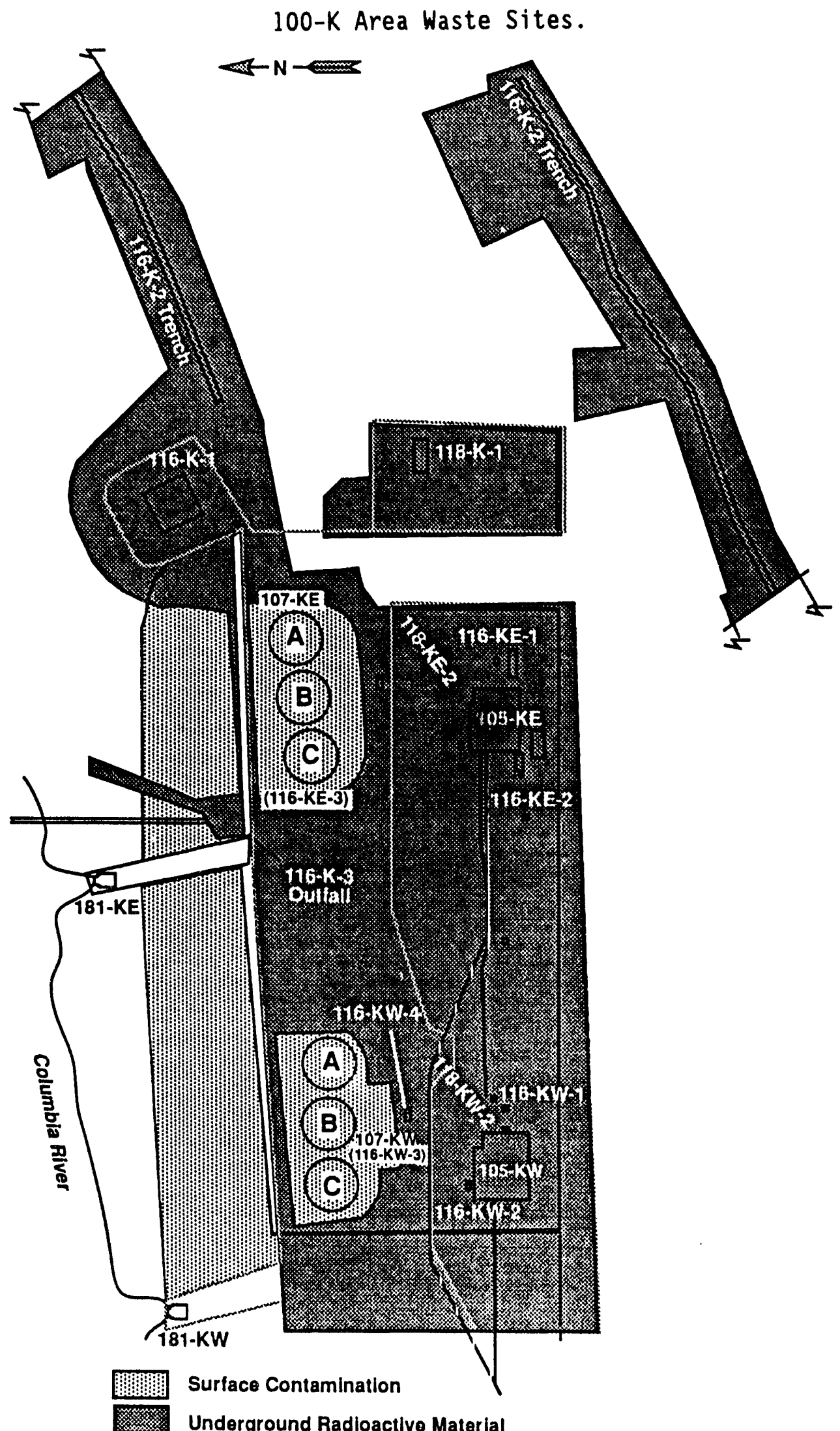

29103008.3 
WHC-SP-0098-5

Rev. 0

Issue Date: 12/15/93

100-N Area Waste Sites.

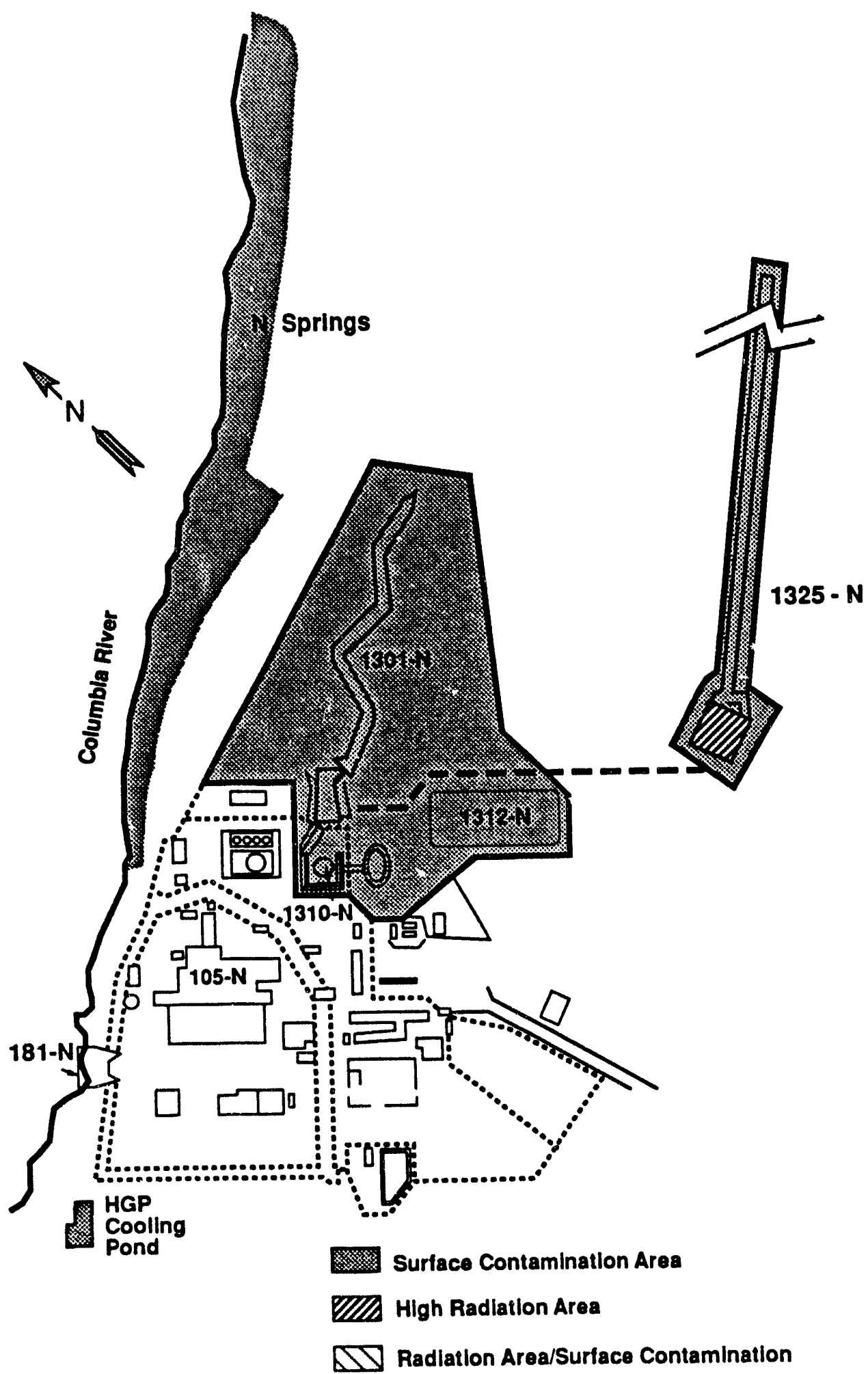

29105057.1 
WHC-SP-0098-5

Rev. 0

Issue Date: $12 / 15 / 93$

100-D Area Waste Sites.

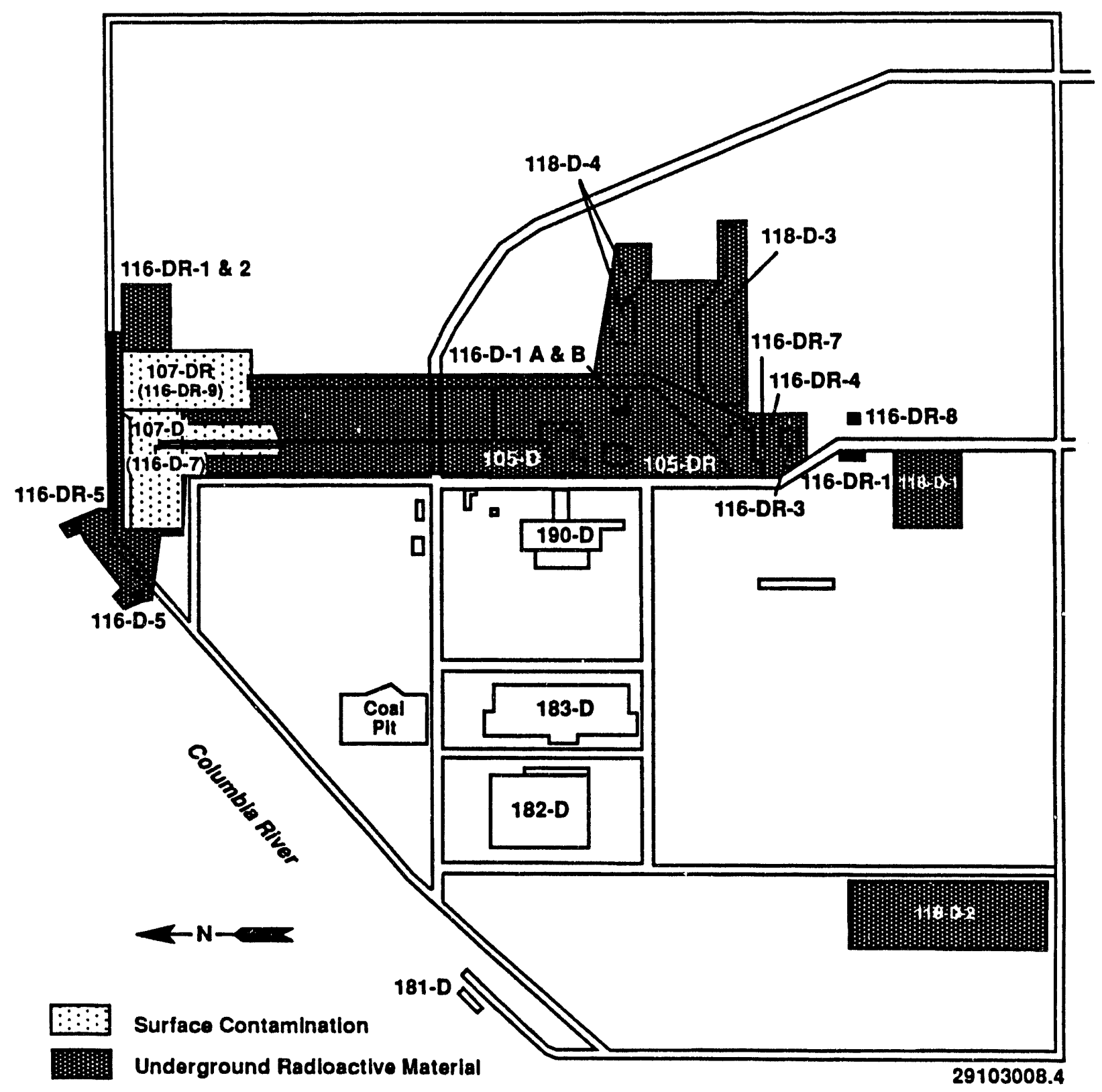


WHC-SP-0098-5

Rev. 0

Issue Date: $12 / 15 / 93$

100-H Area Waste Sites.

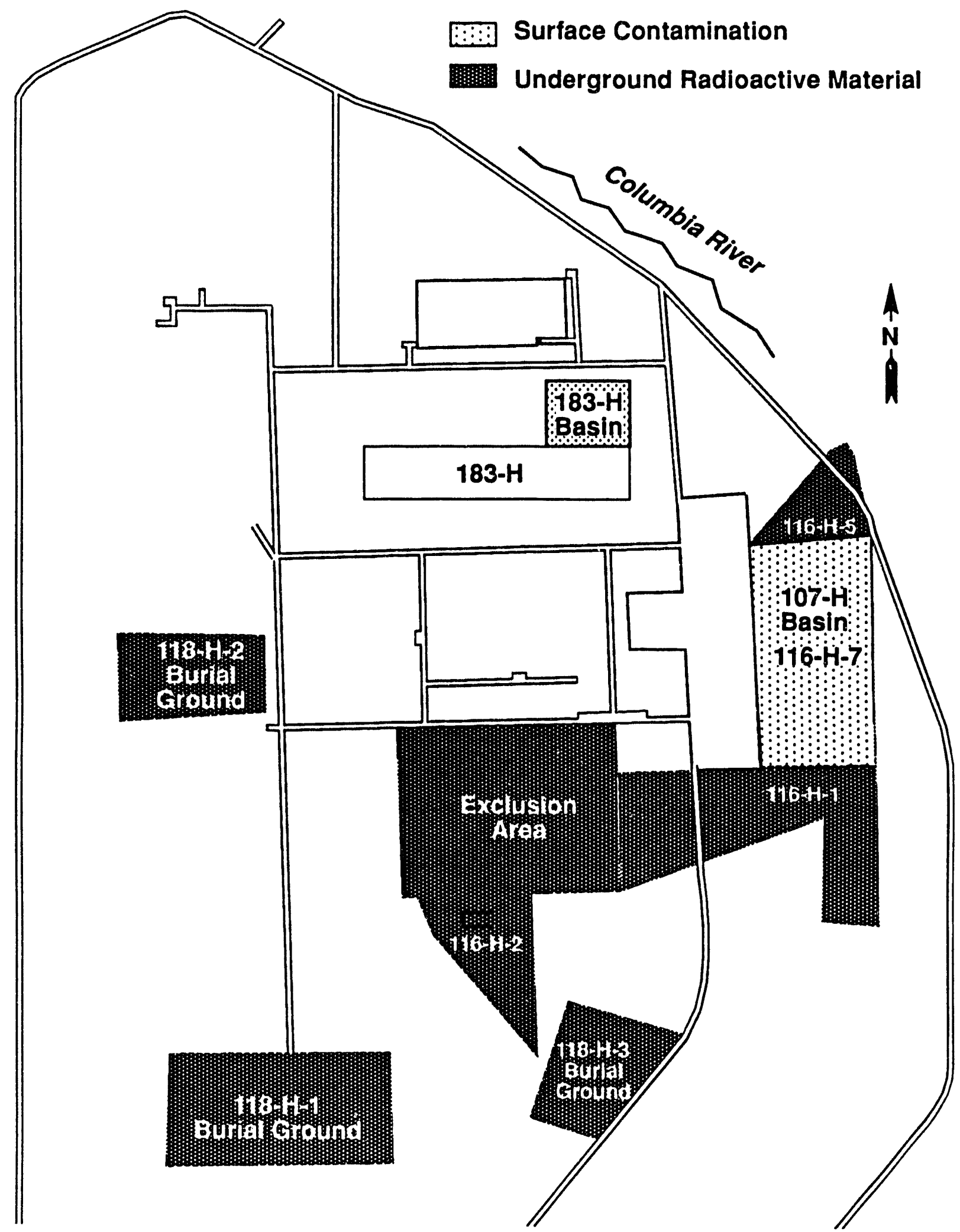

29103008.1 
WHC-SP-0098-5

Rev. 0

Issue Date: 12/15/93

100-F Area Waste Sites.

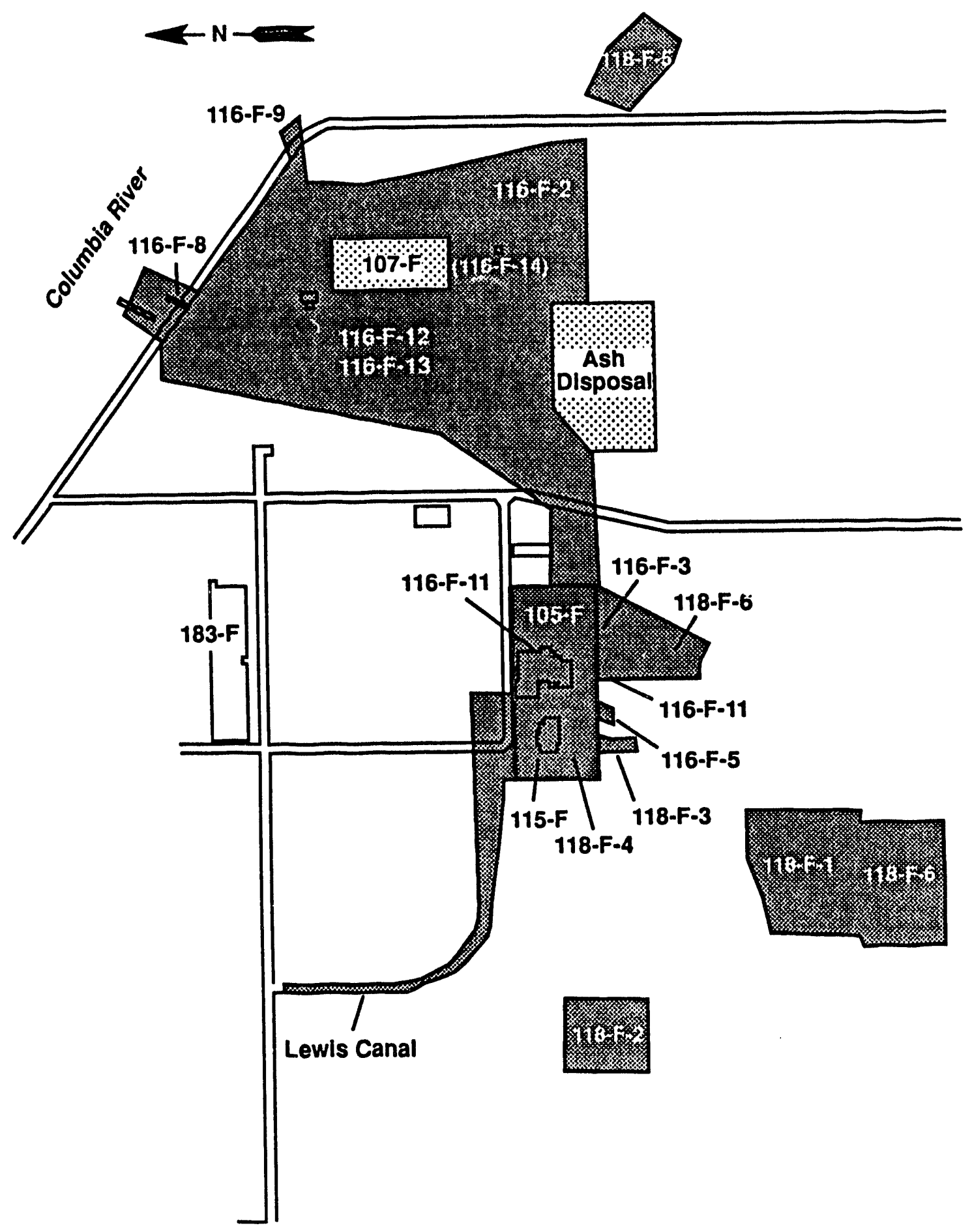

3 Surface Contamination

Underground Radioactive Material

29103008.5 


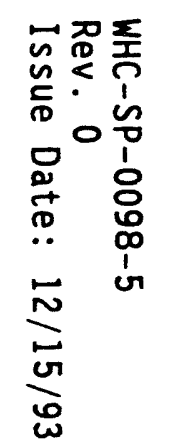


WHC-SP-0098-5

Rev. 0

Issue Date: $12 / 15 / 93$

200 East Area Waste Sites.

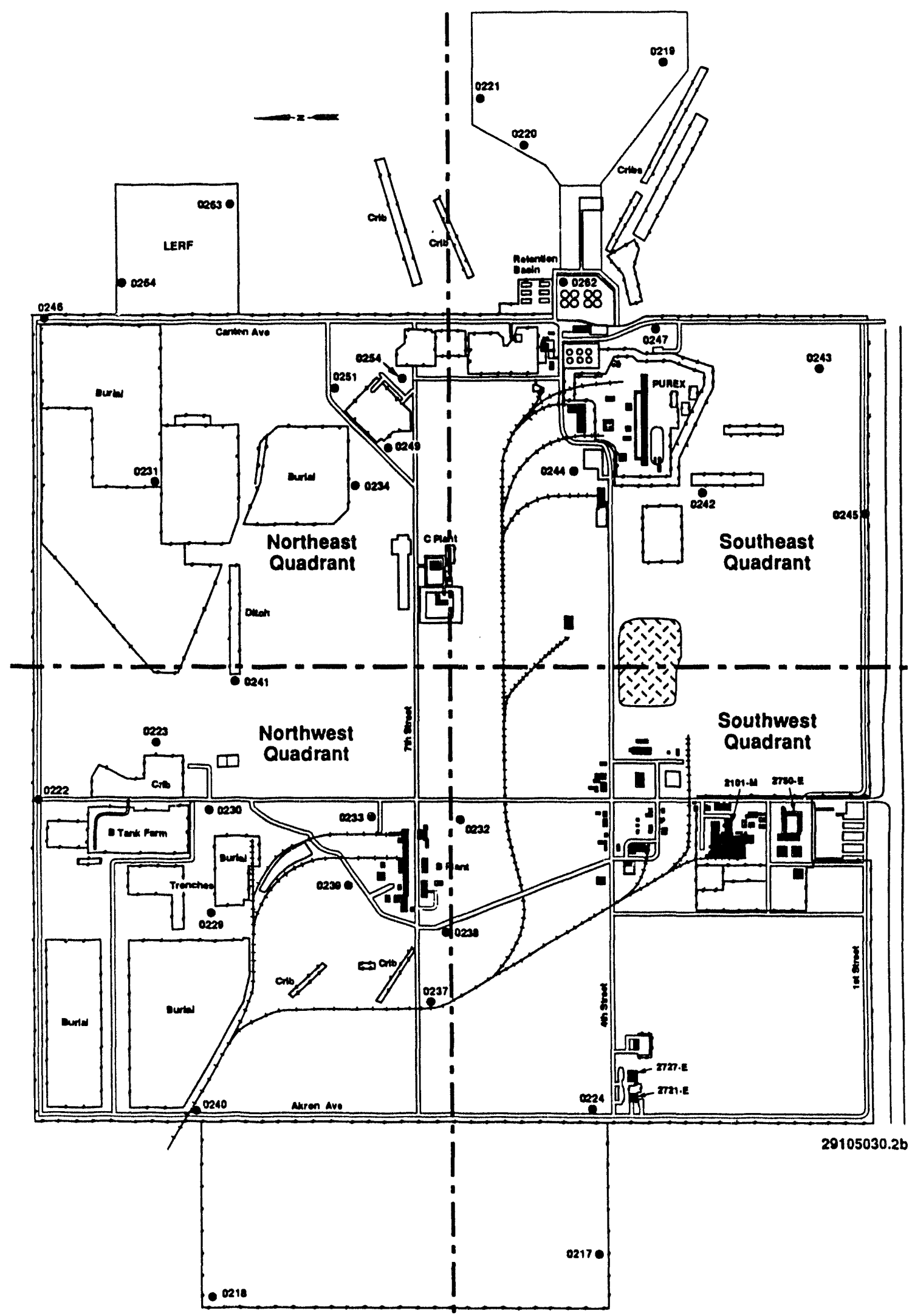




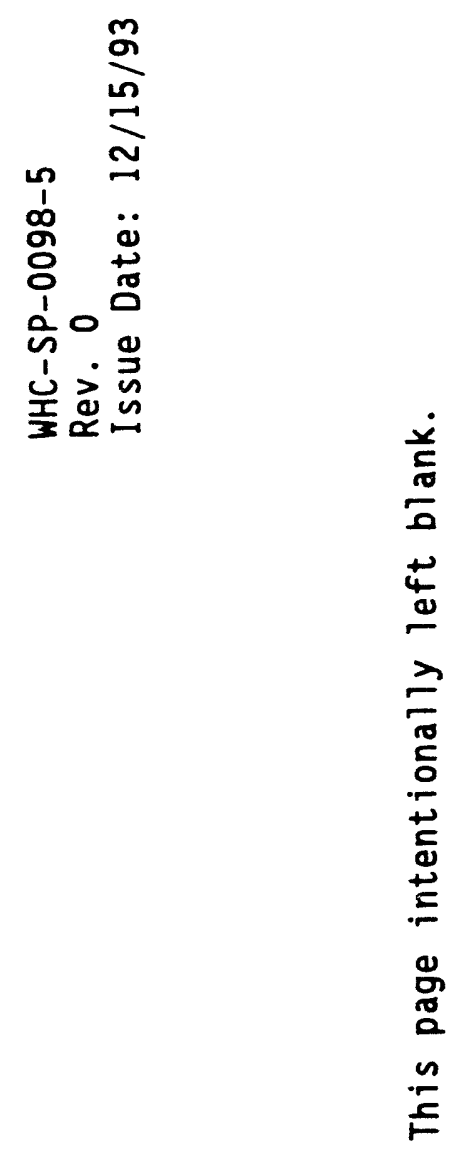


WHC-SP-0098-5

Rev. 0

Issue Date: 12/15/93

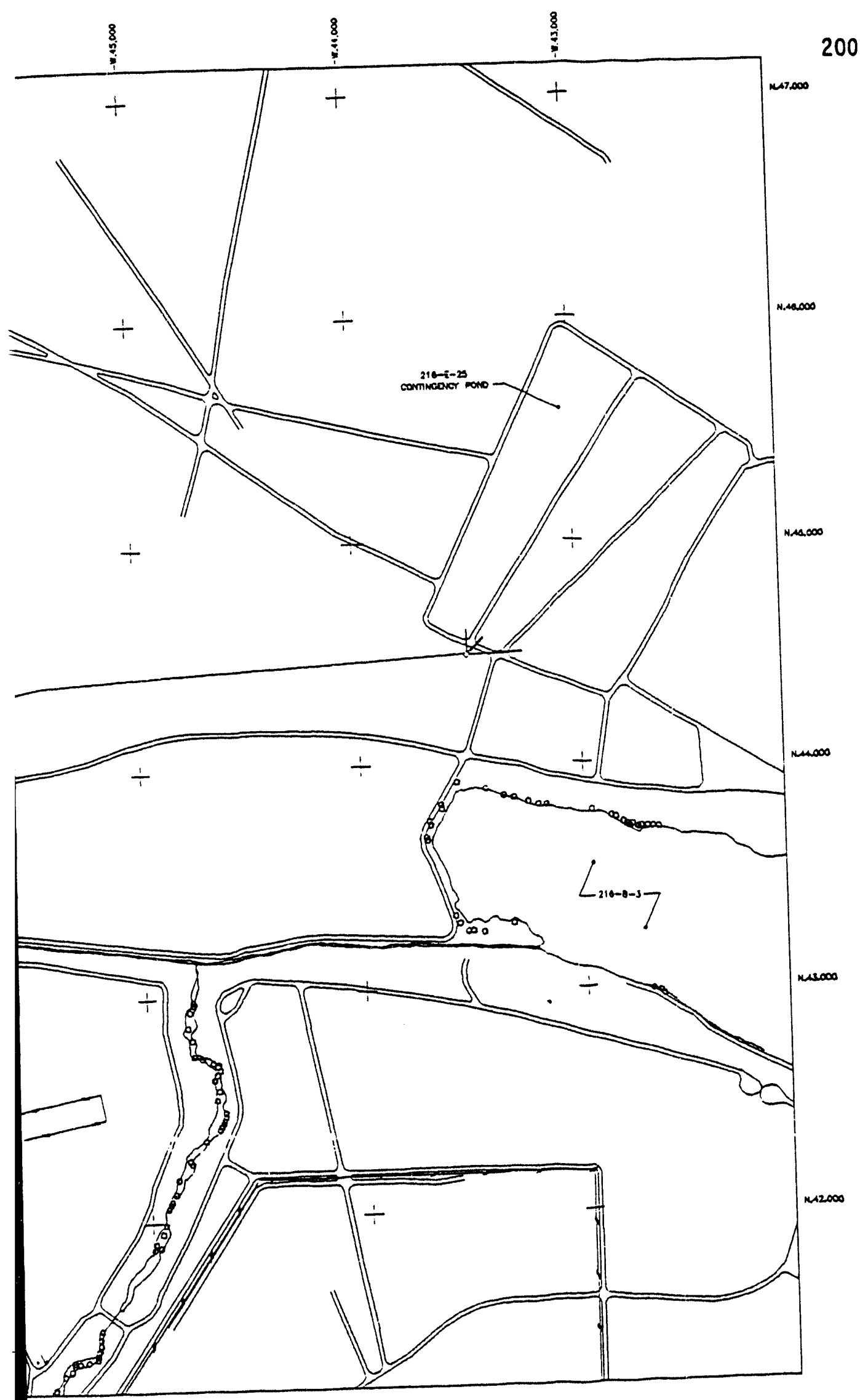




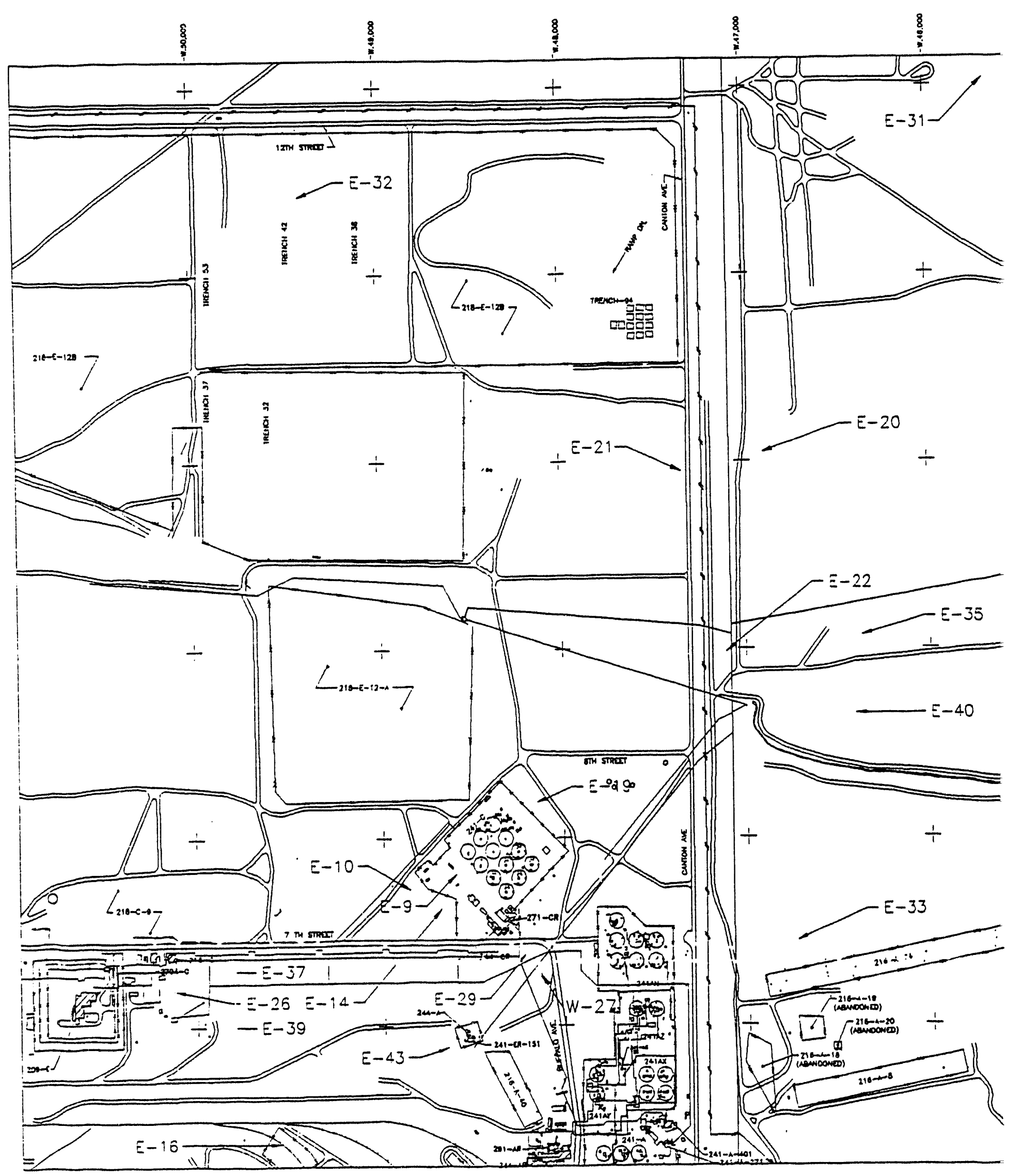




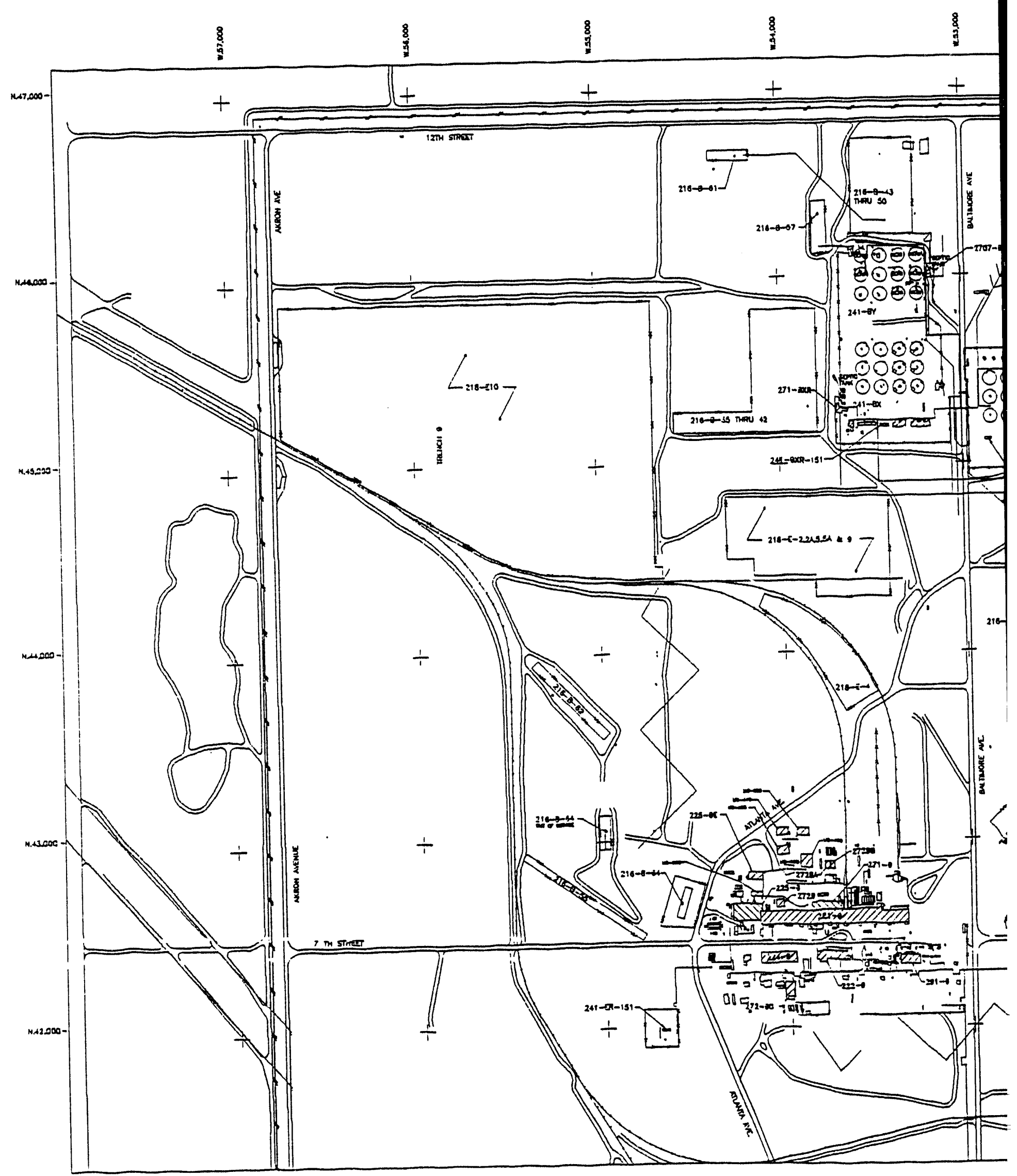




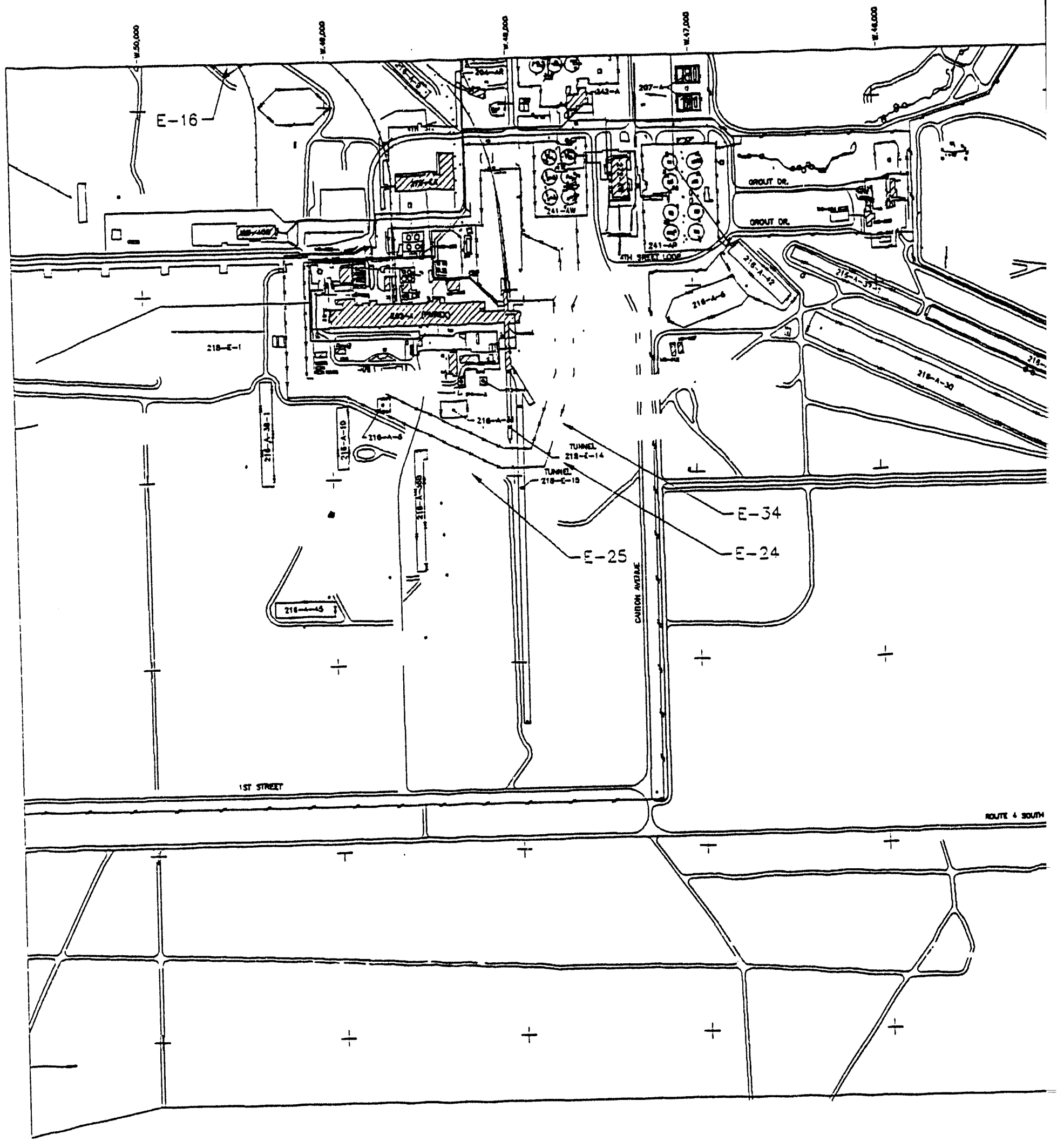


WHC-SP-0098-5

Rev. 0

Issue Date: $12 / 15 / 93$

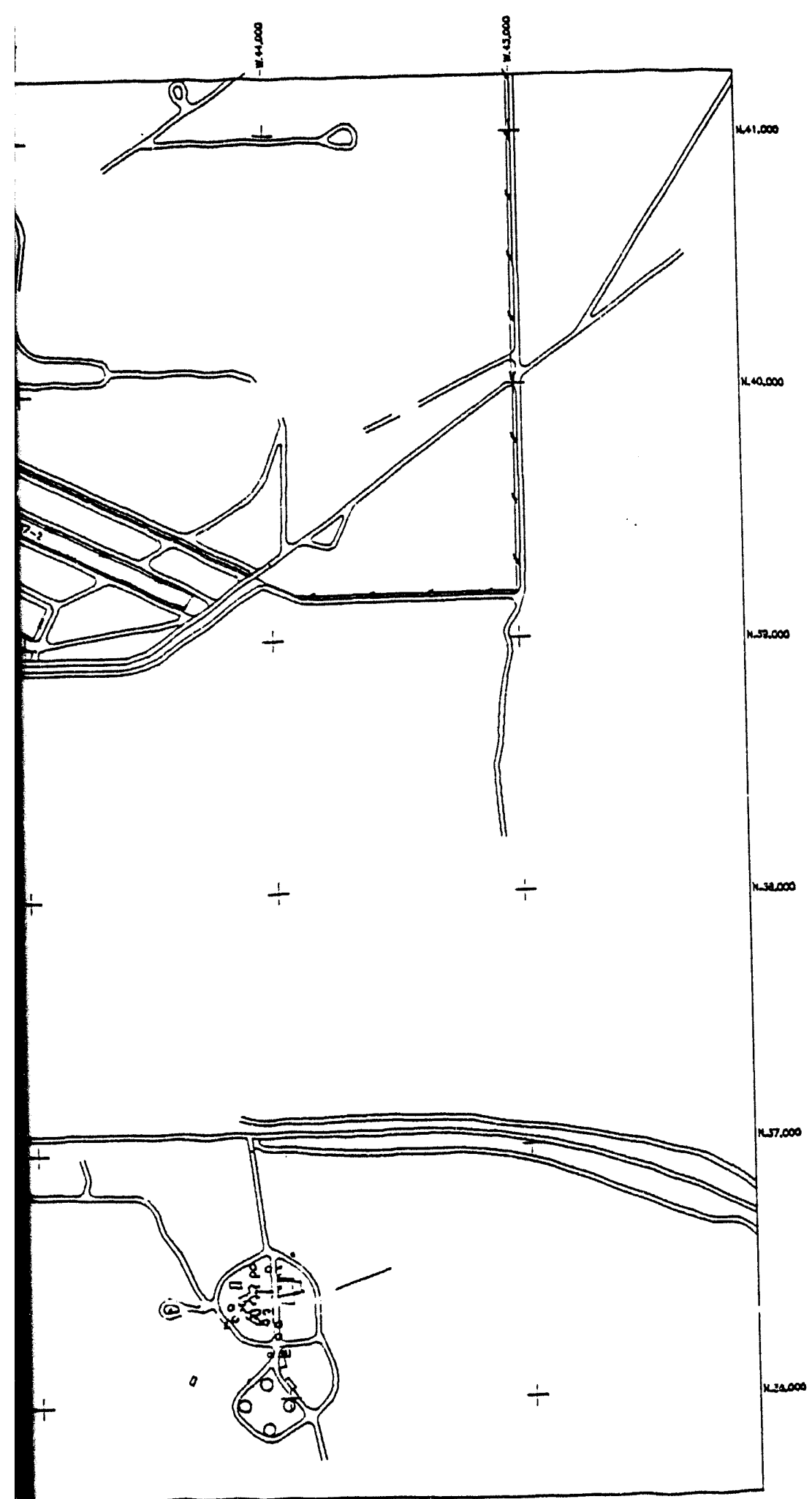

200 East Area Waste Sites, S.E. Quadrant. 


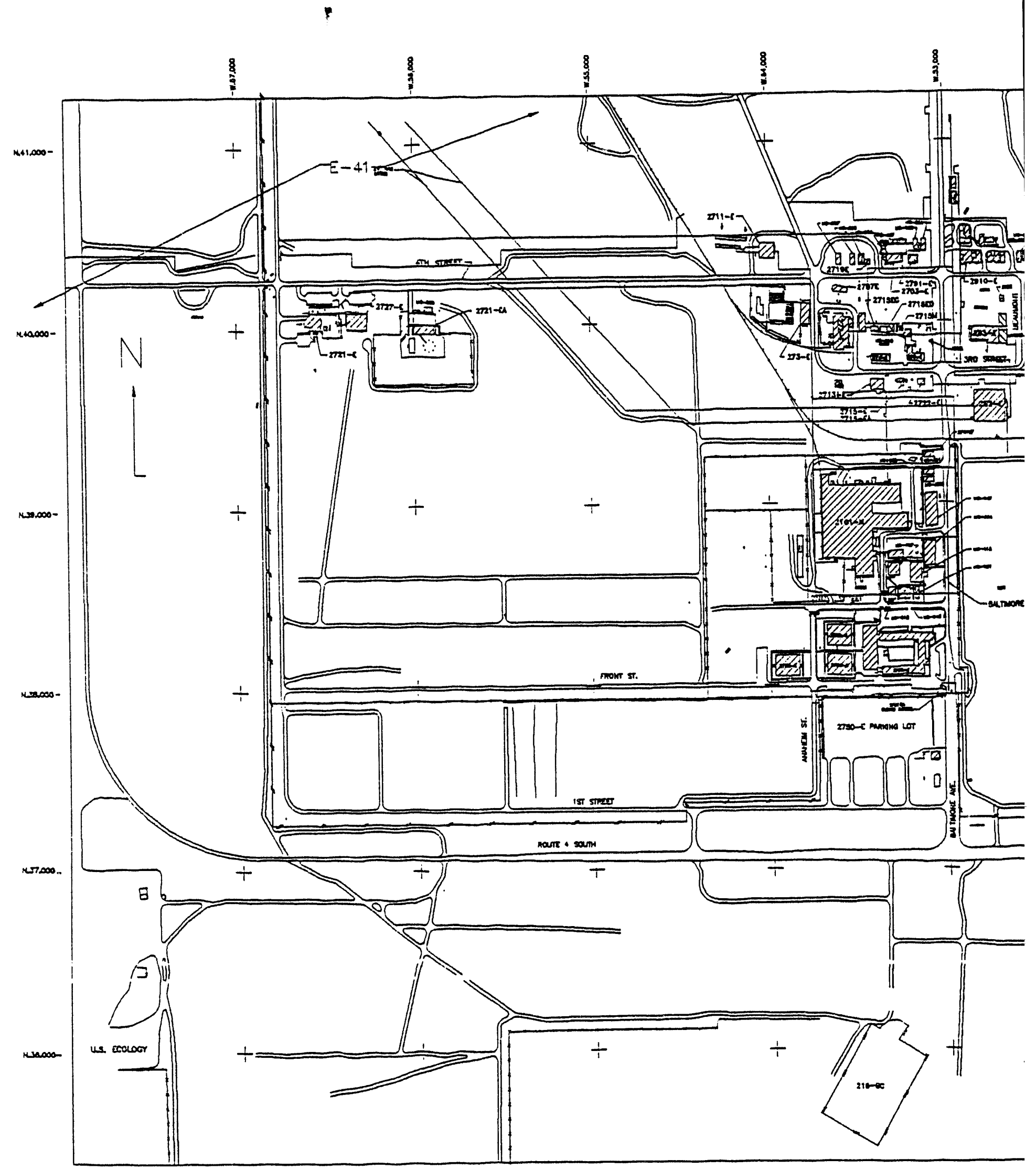


WHC-SP-0098-5

Rev. 0

Issue Date: $12 / 15 / 93$

200 West Area Waste Sites.

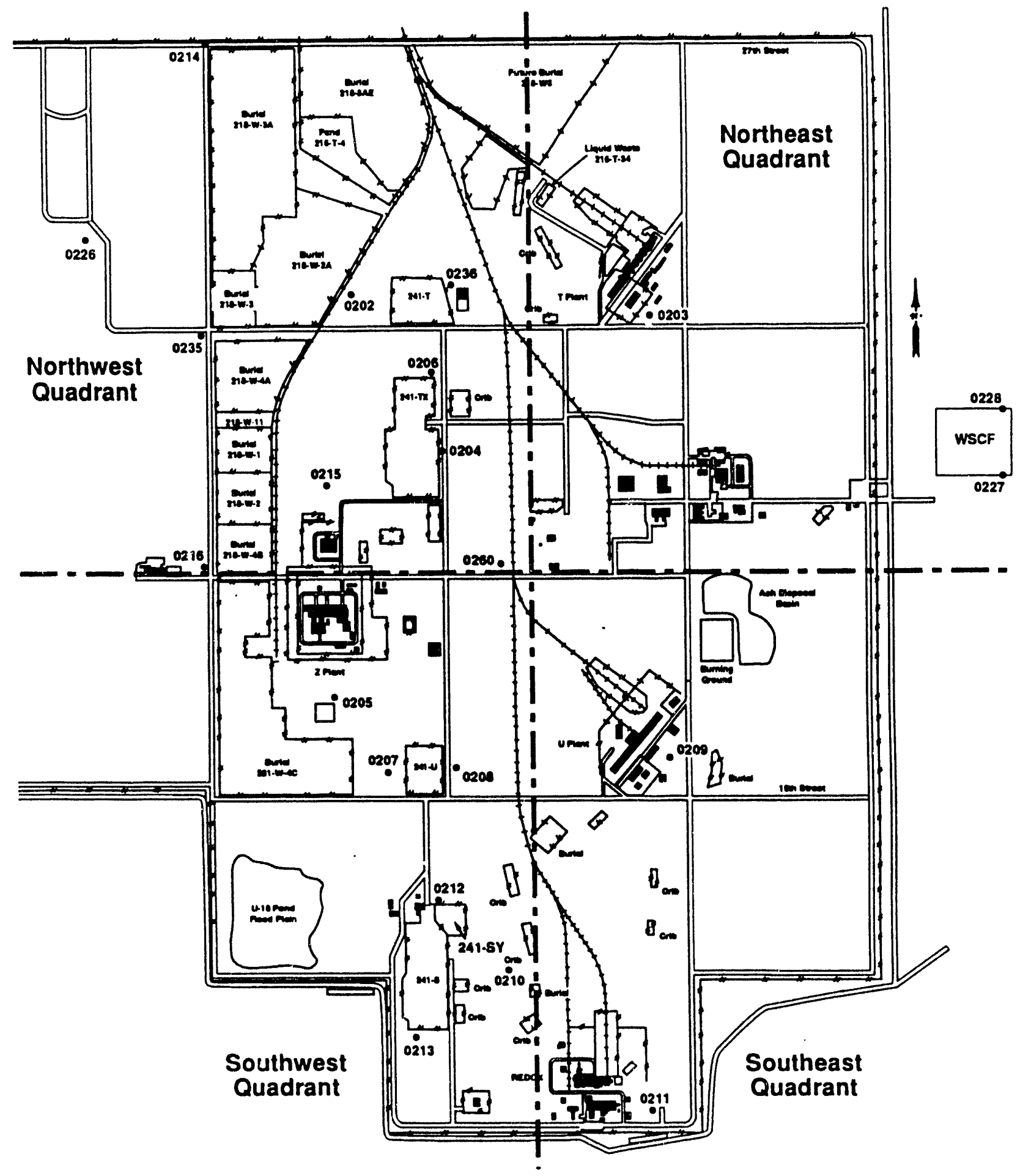

29105030.30 
WHC-SP-0098-5

Rev. 0

Issue Date: 12/15/93

This page intentionally left blank. 


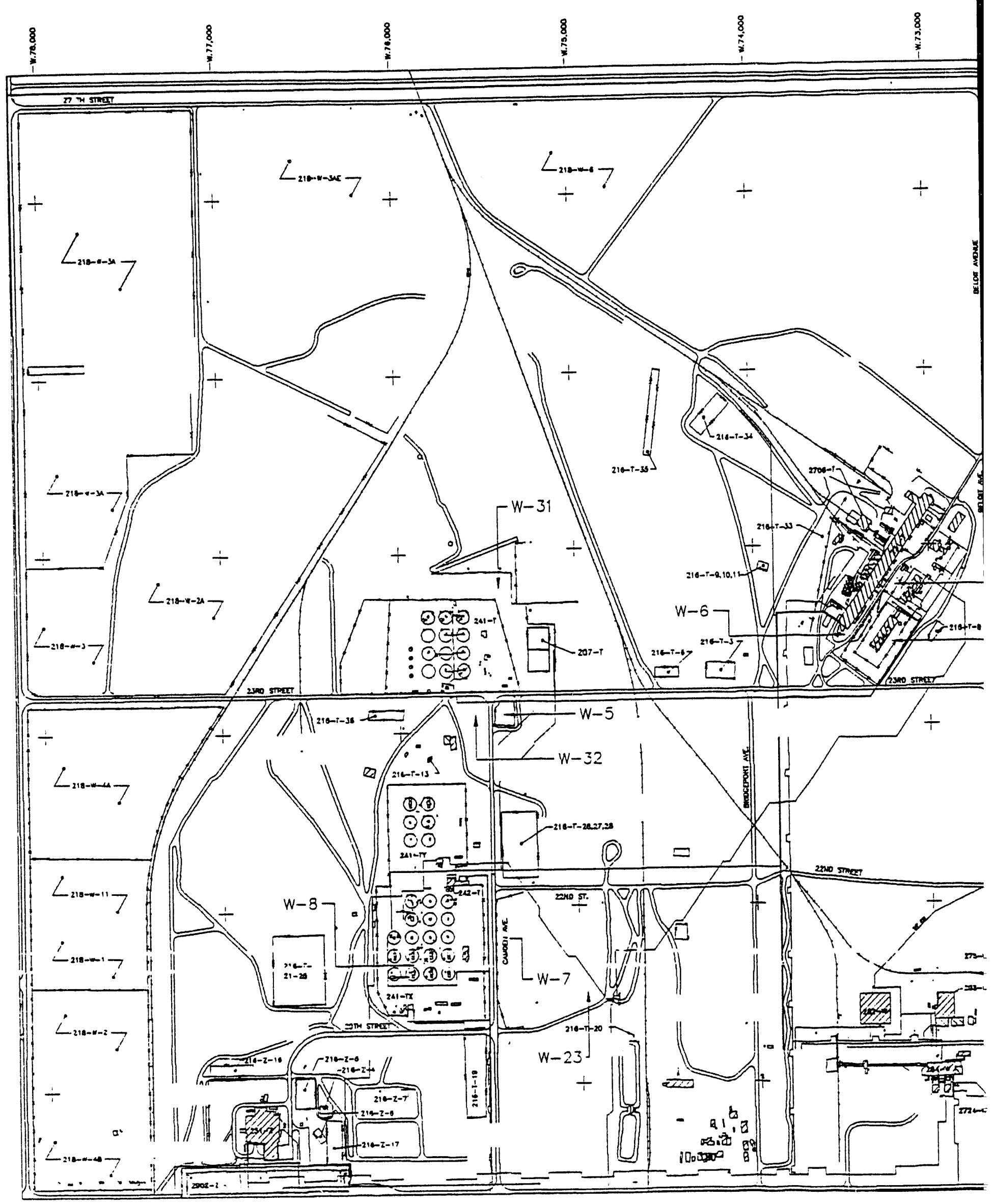


WHC-SP-0098-5

Rev. 0

Issue Date: $12 / 15 / 93$

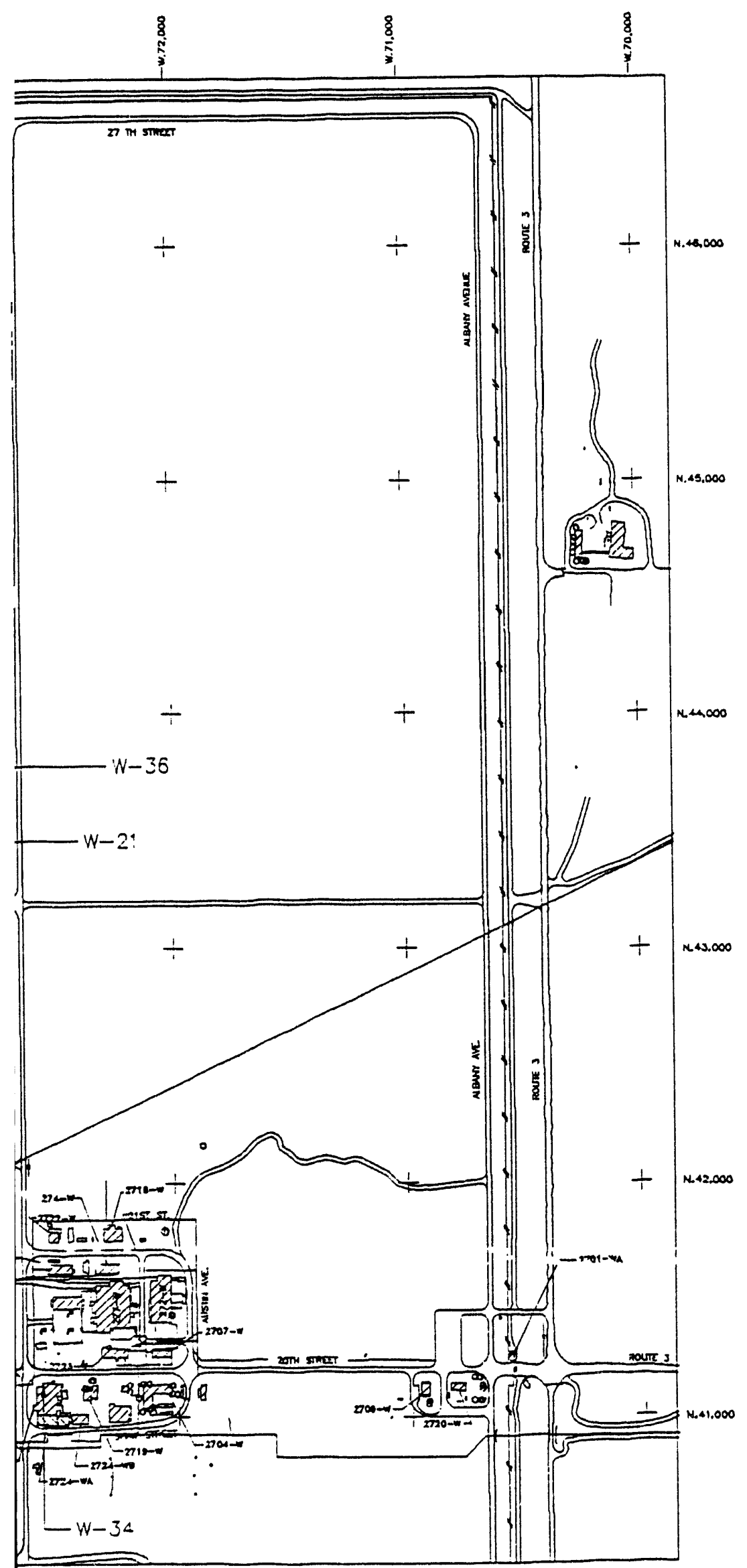

\section{West Area Waste Sites,}

N.E. Quadrant. 


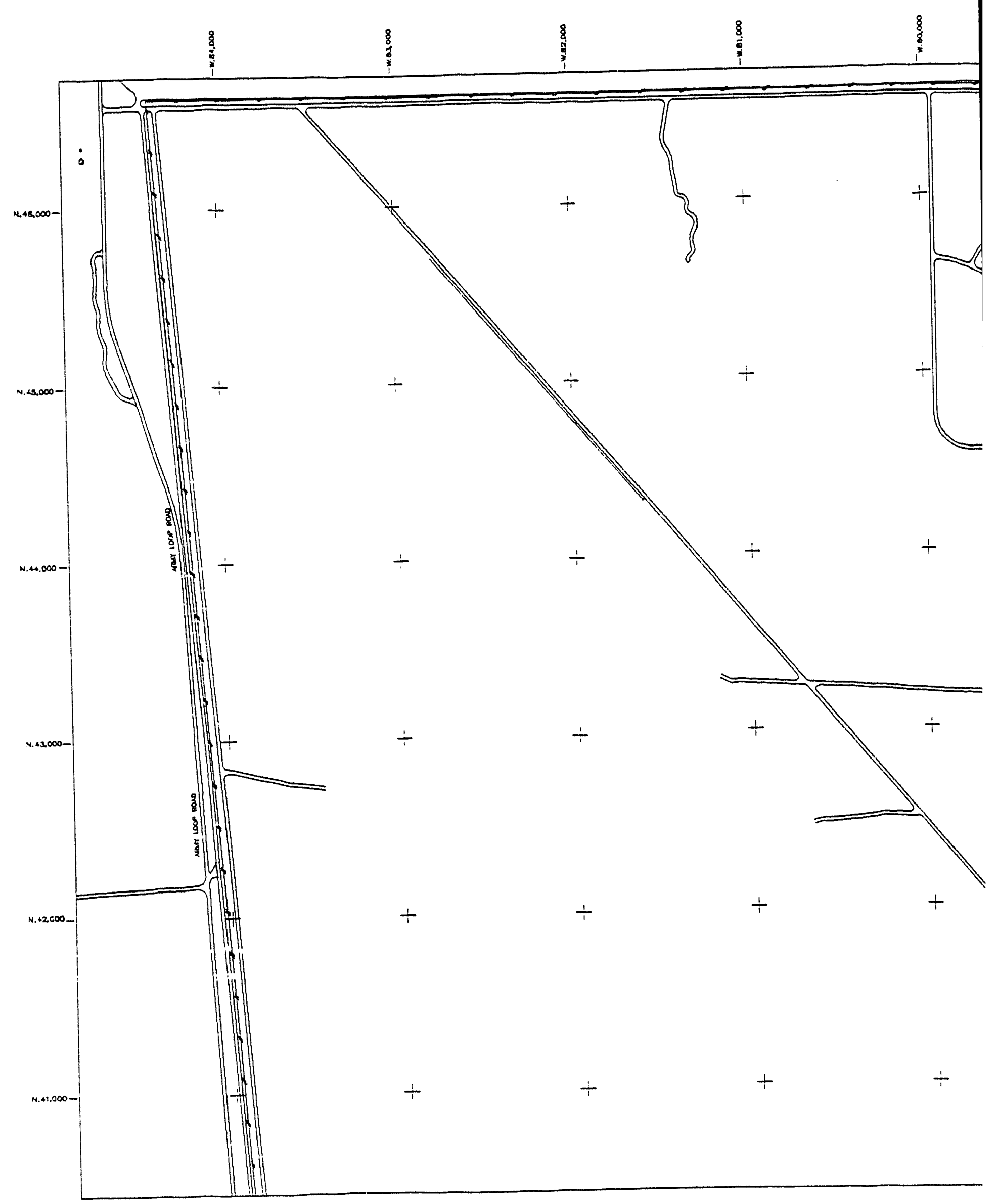




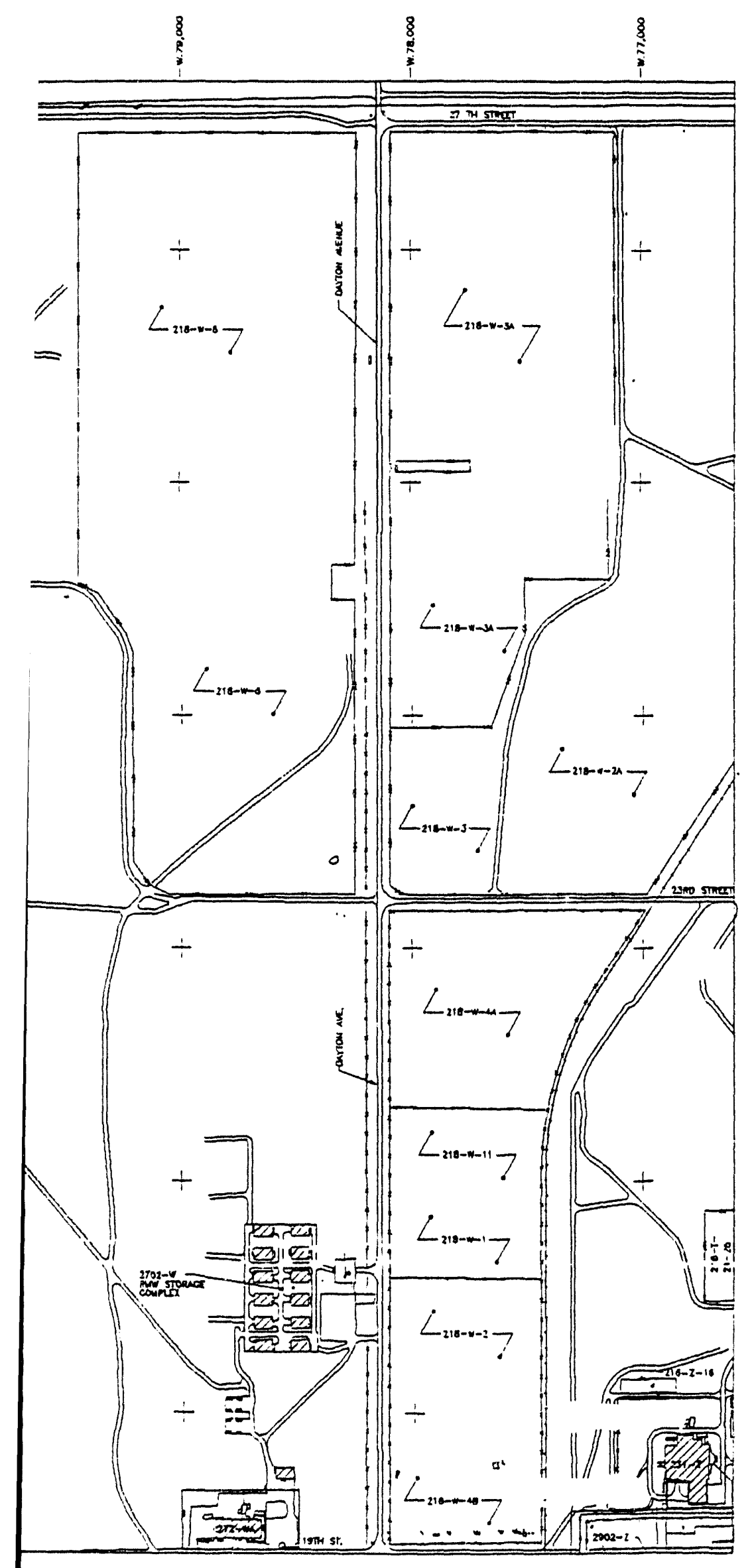

WHC-SP-0098-5

Rev. 0

Issue Date: 12/15/93

200 West Area Waste Sites, N.W. Quadrant. 


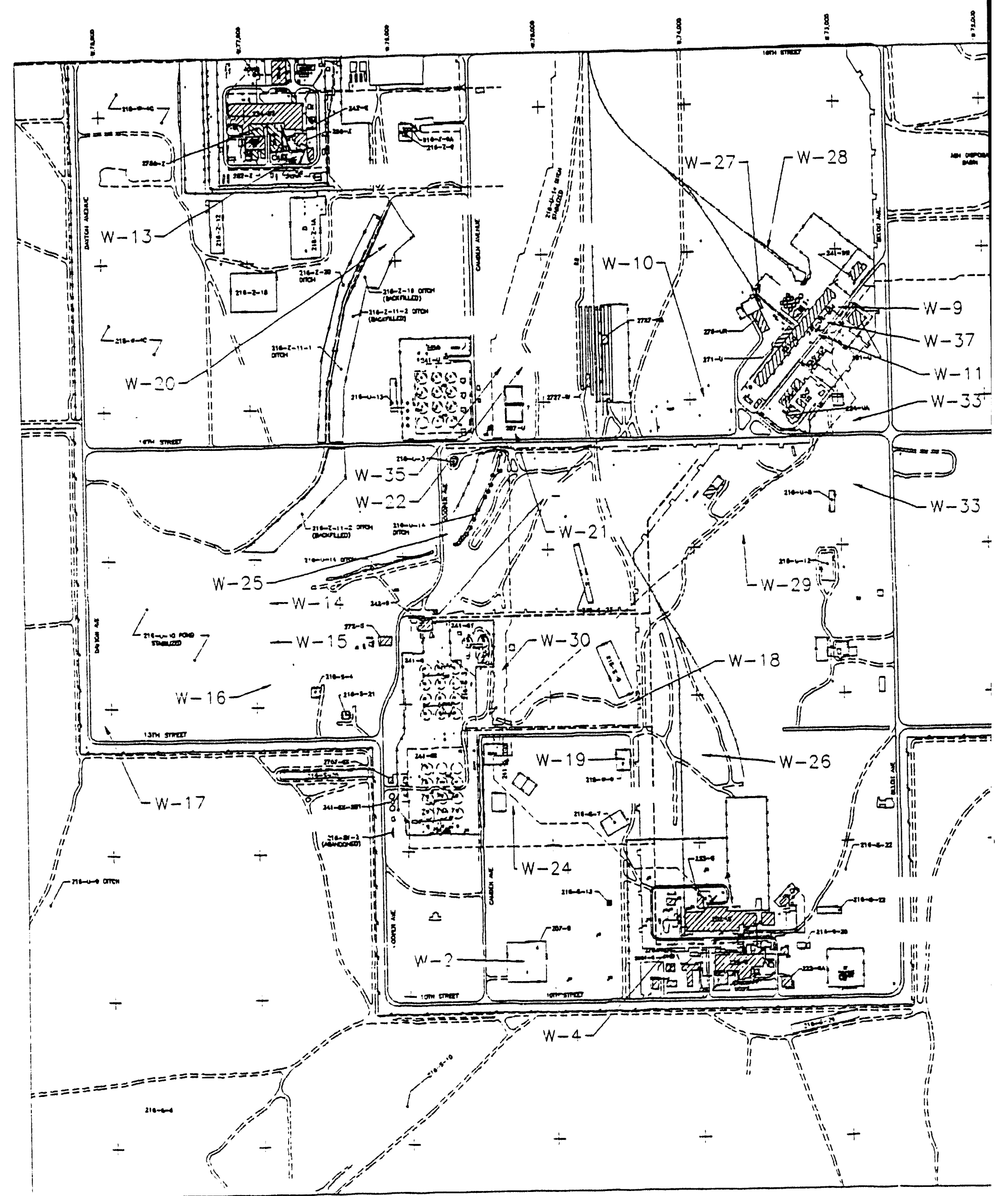


WHC-SP-0098-5

Rev. 0

Is sue Date: 12/15/93

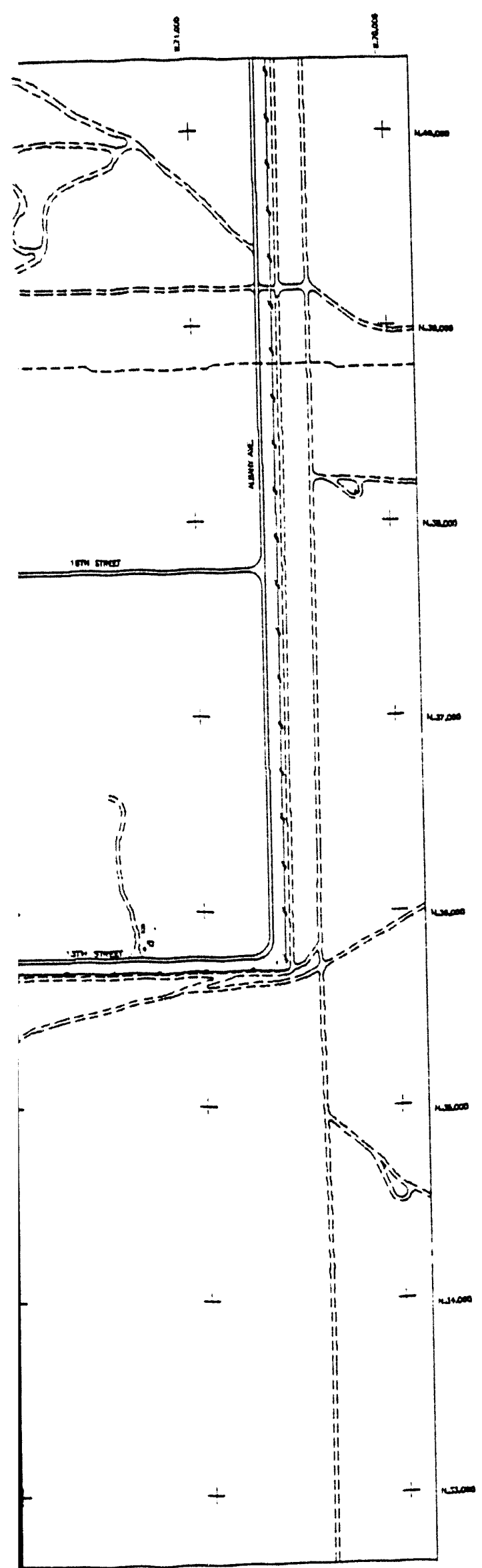

200 West Area Waste Sites, S.E. Quadrant. 


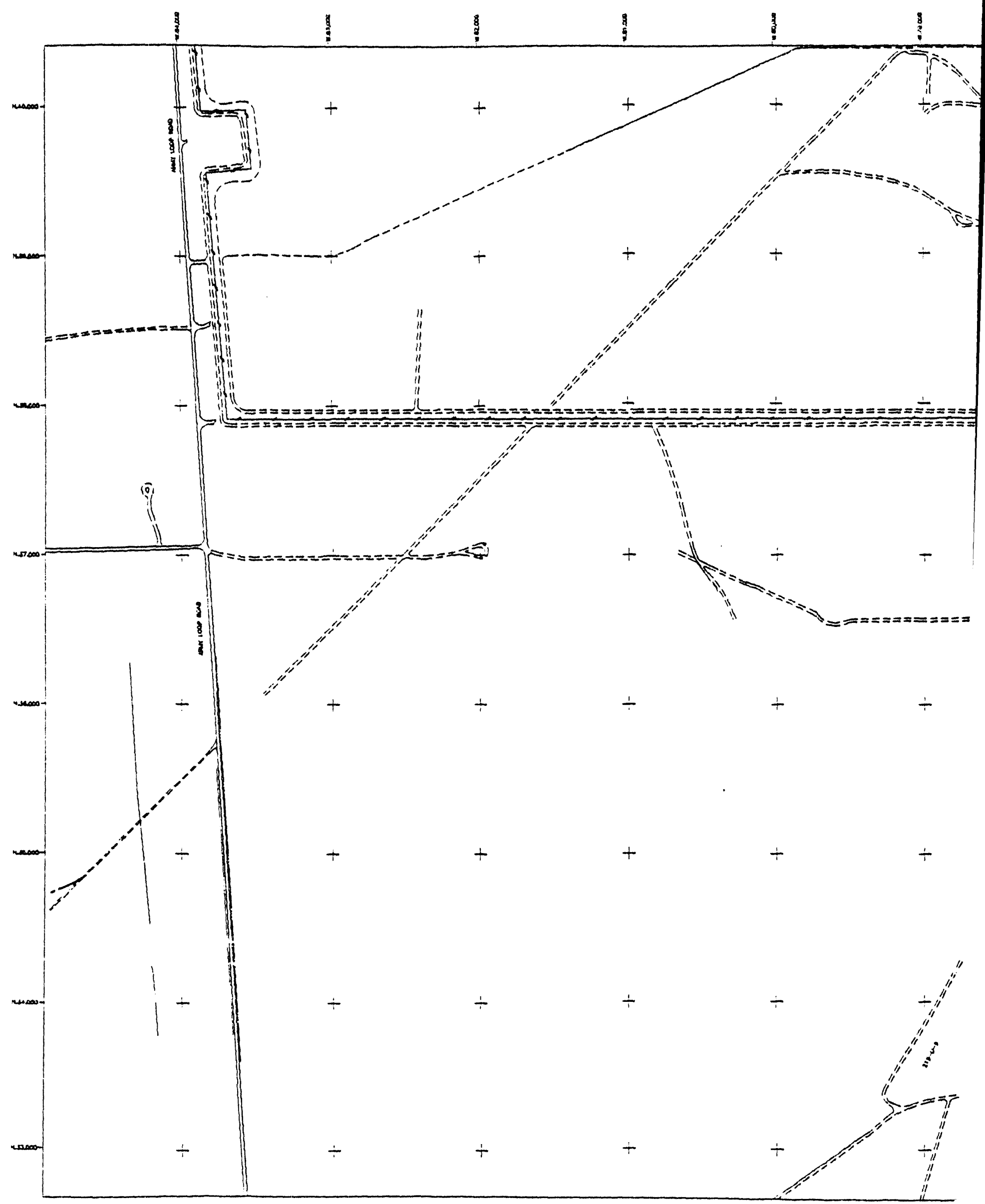




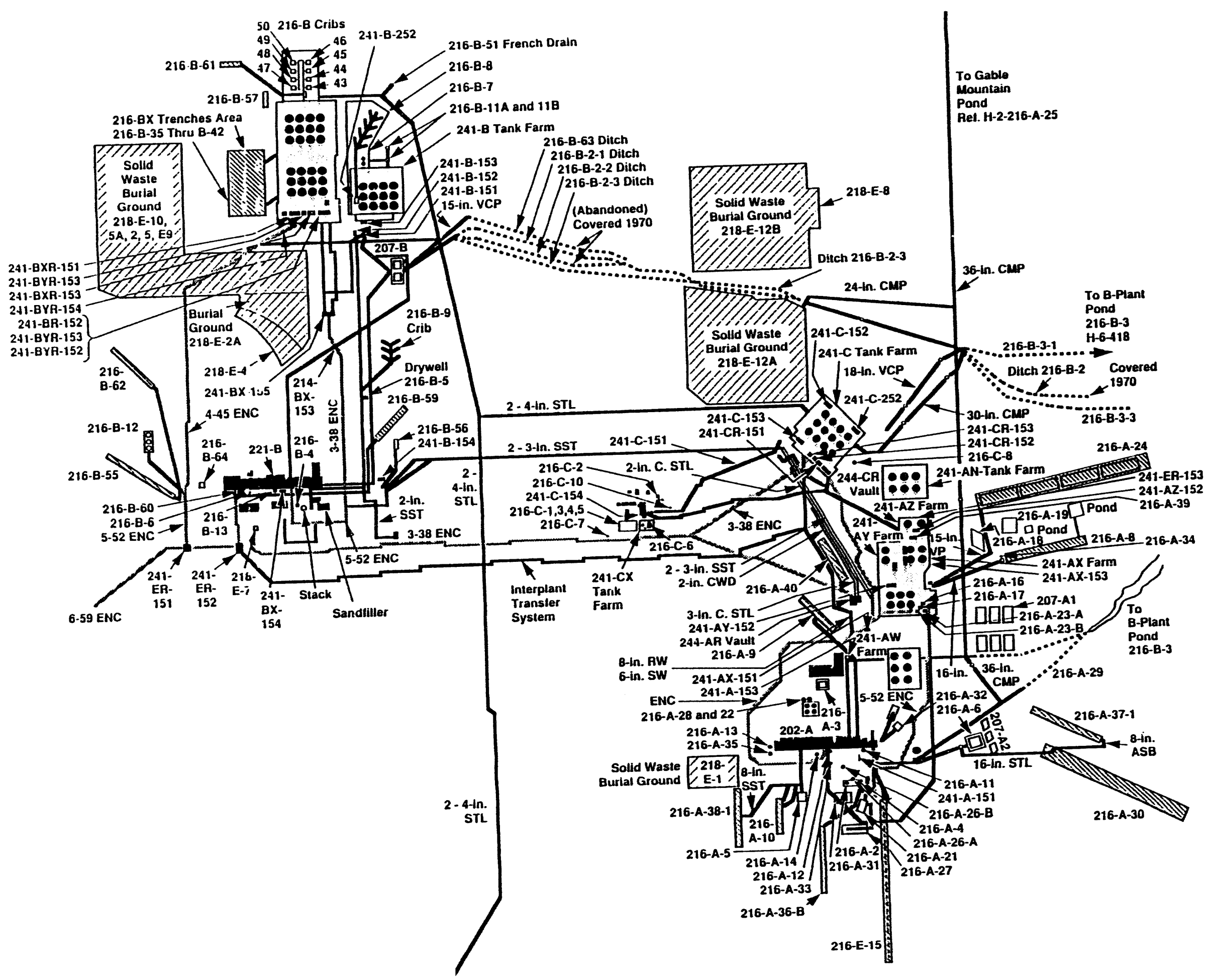




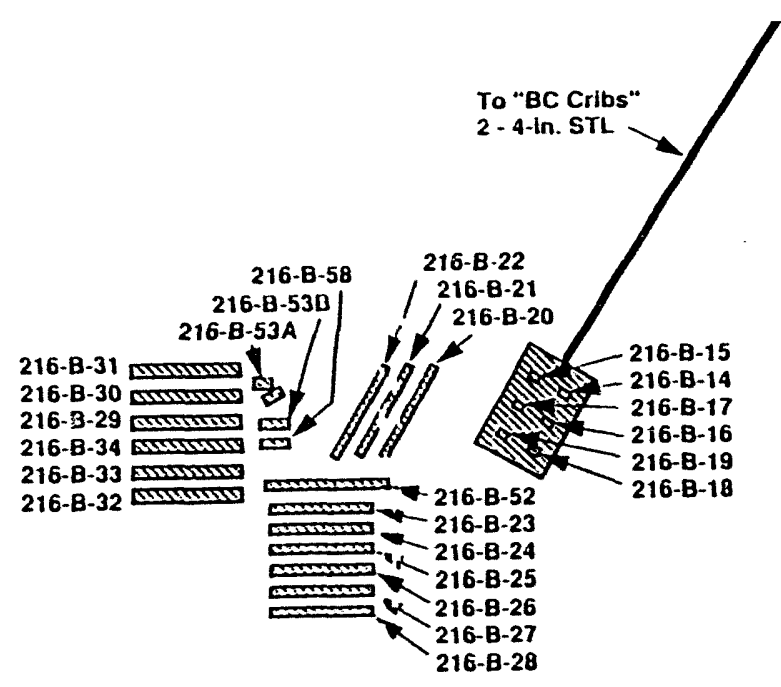

Notes:

1) All Facilities are Radioactive Unless Marked Otherwise.
DI7 218 - Solid Waste Burial Site

216 - Liquid Waste Burial Site

Trench

- Dry Well or French Drain

8: 241 - Tank Farm

Encasement

Pipe Line

NC Not Contaminated

vcP Vitrified Clay Pipe
1

H9310036.1 


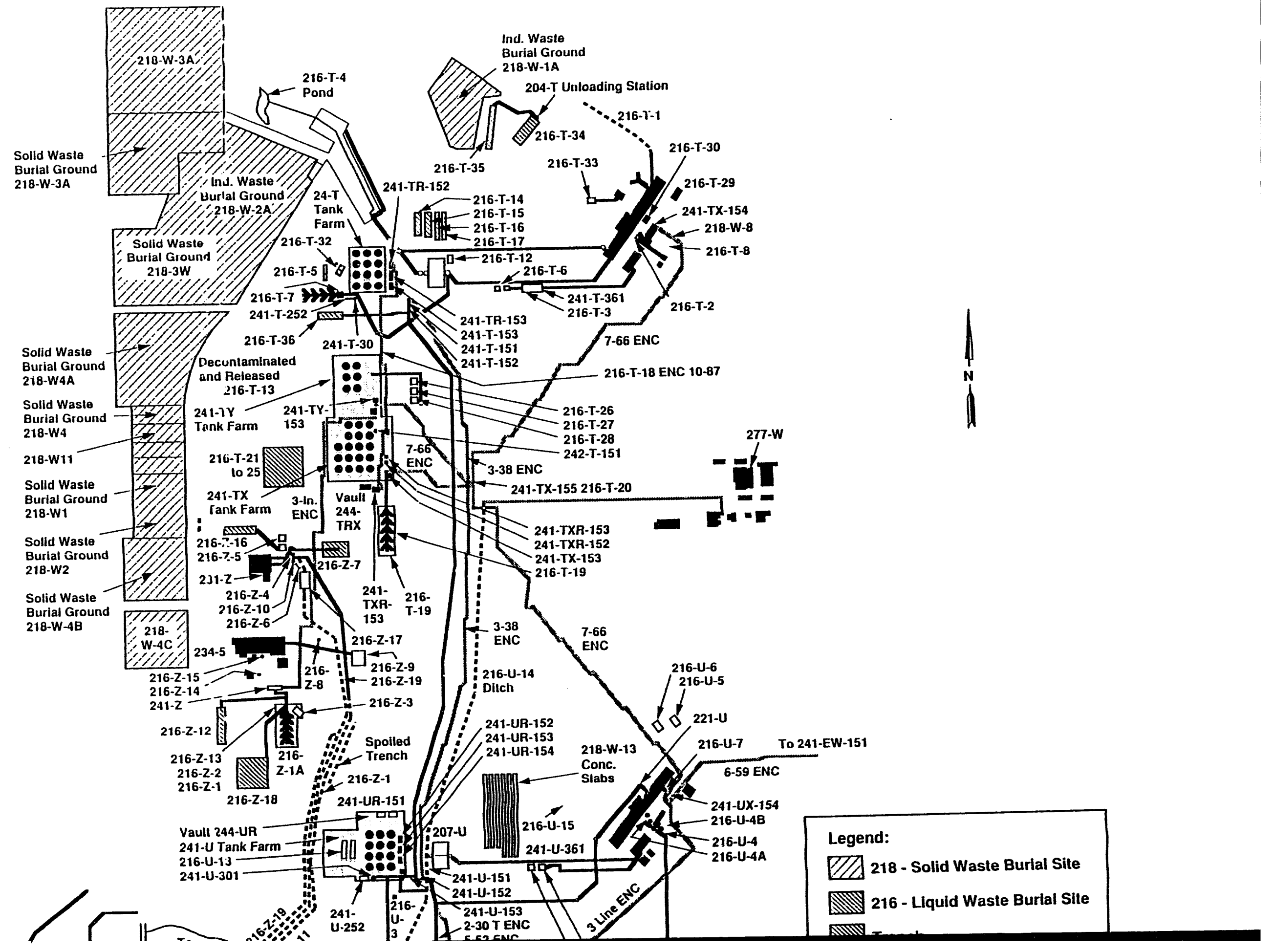




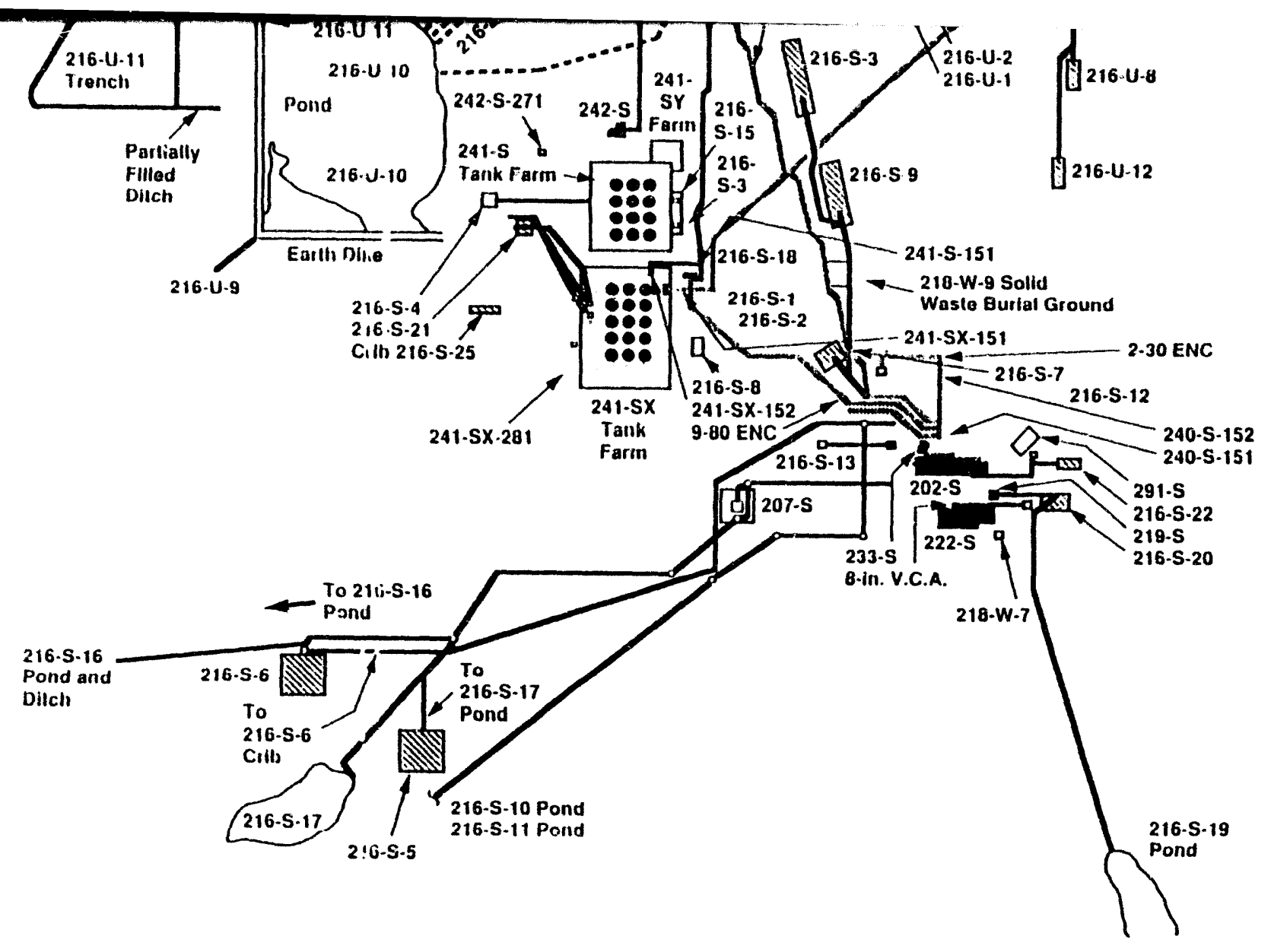

- Dry Well or French Drain

[8: 241 - Tank Farm

Encasement

- Pipe Line

VCP Vitrilled Clay Pipe

M9310036.2 
WHC-SP-0098-5

Rev. 0

Issue Date: $12 / 15 / 93$

300 Area Waste Sites.

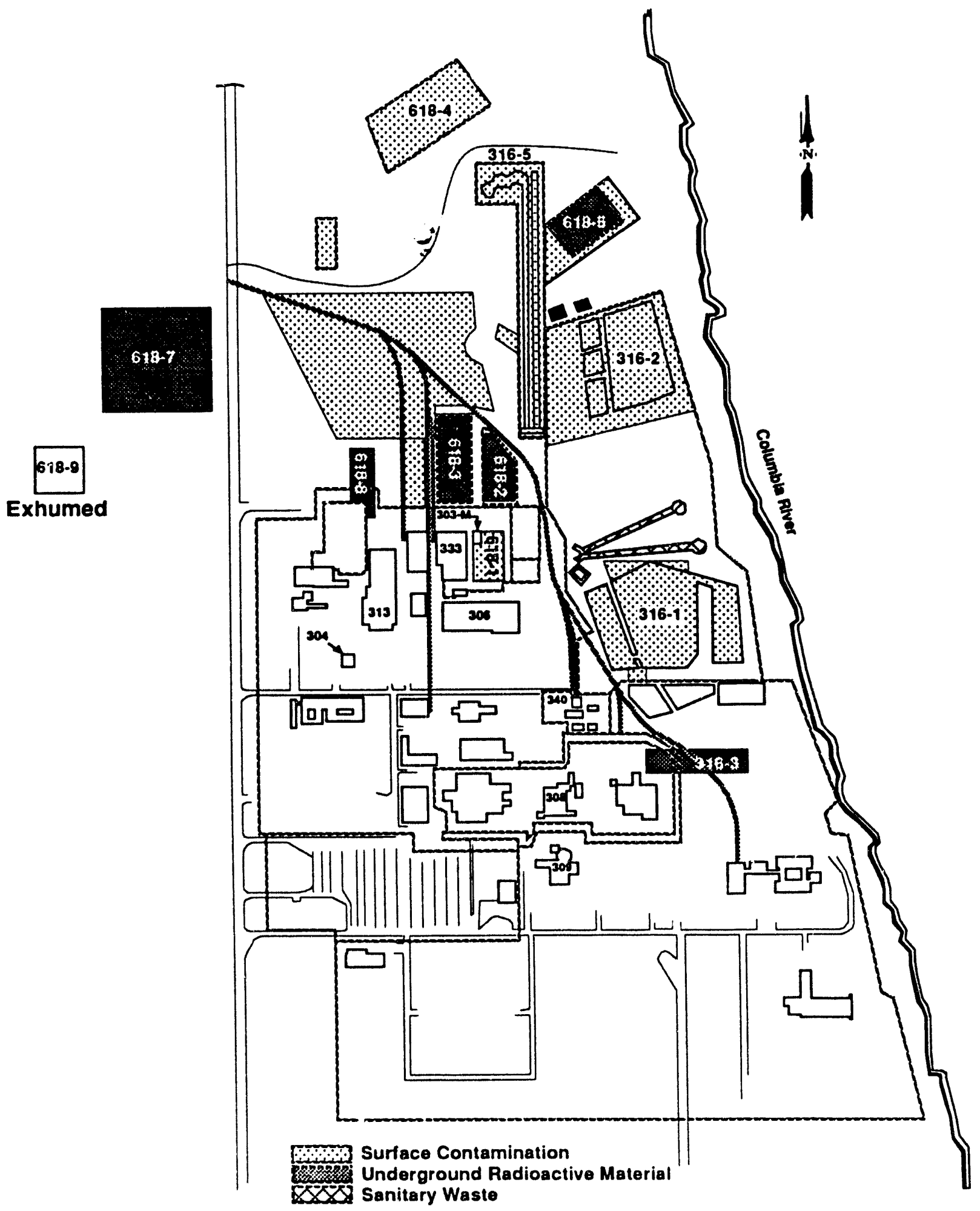

29103008.8 
WHC-SP-0098-5

Rev. 0

Issue Date: 12/15/93

400 Area Waste Sites.
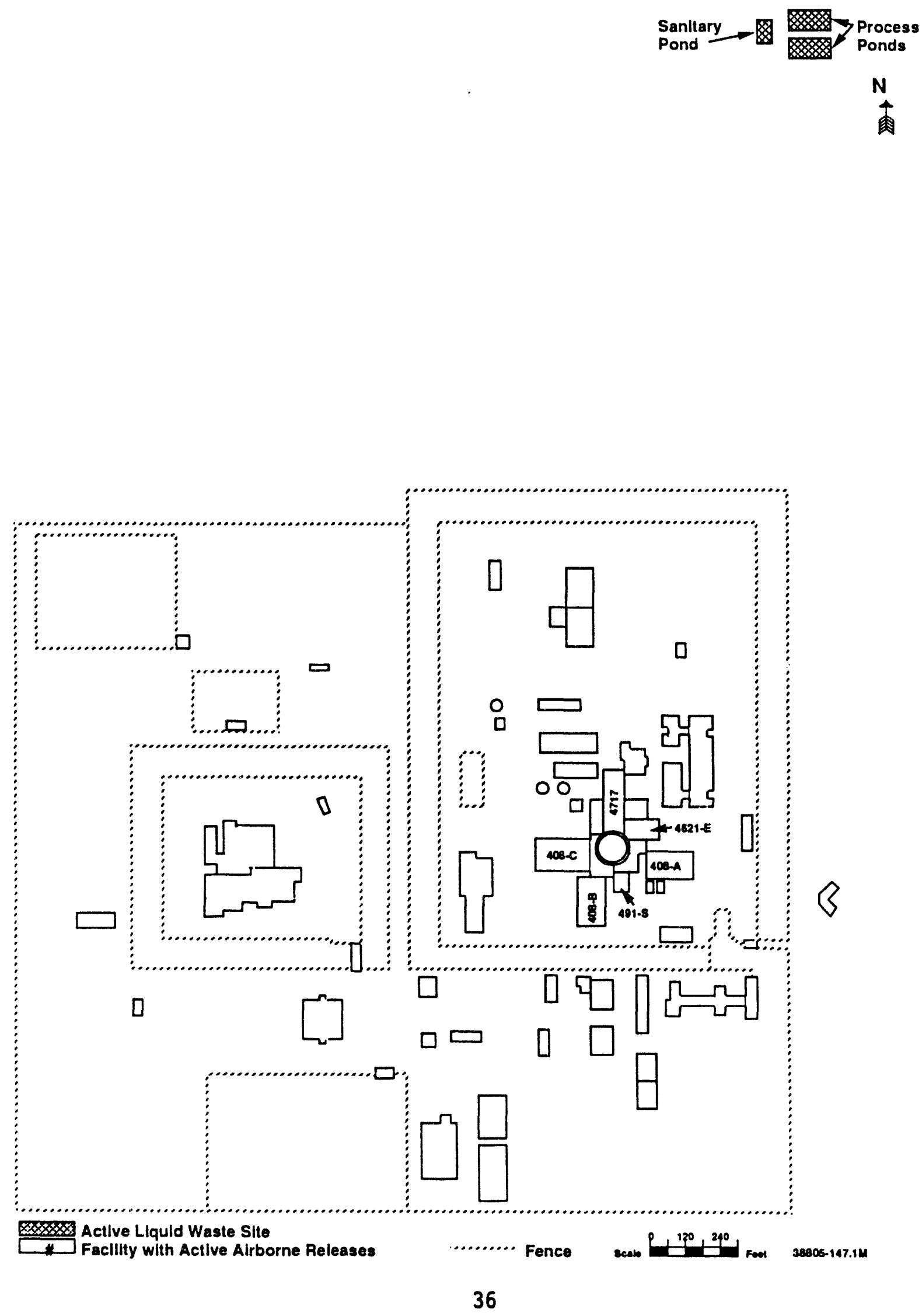
WHC-SP-0098-5

Rev. 0

Issue Date: 12/15/93

600 Area Waste Sites.

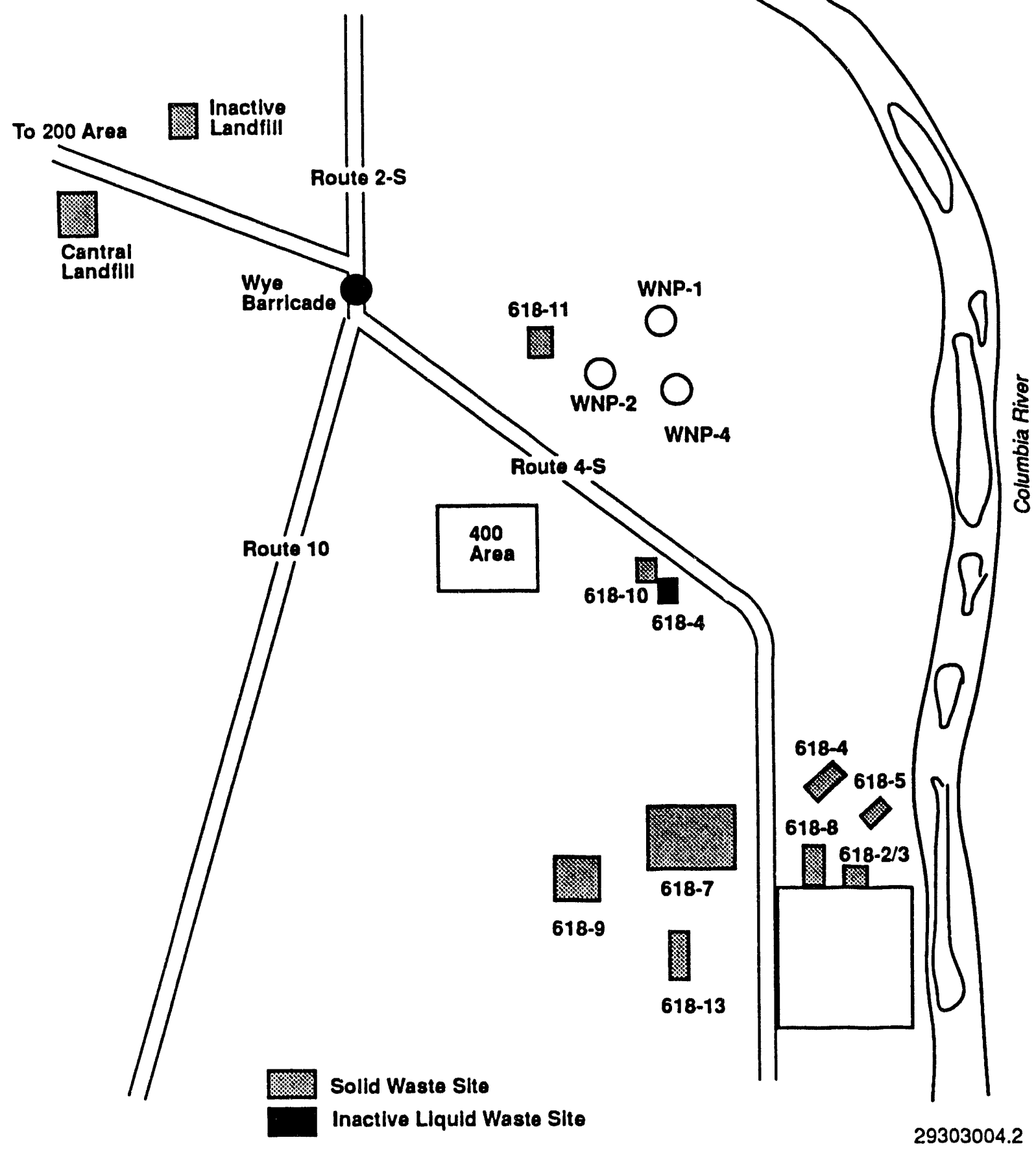


WHC-SP-0098-5

Rev. 0

Issue Date: 12/15/93

Site Highways.

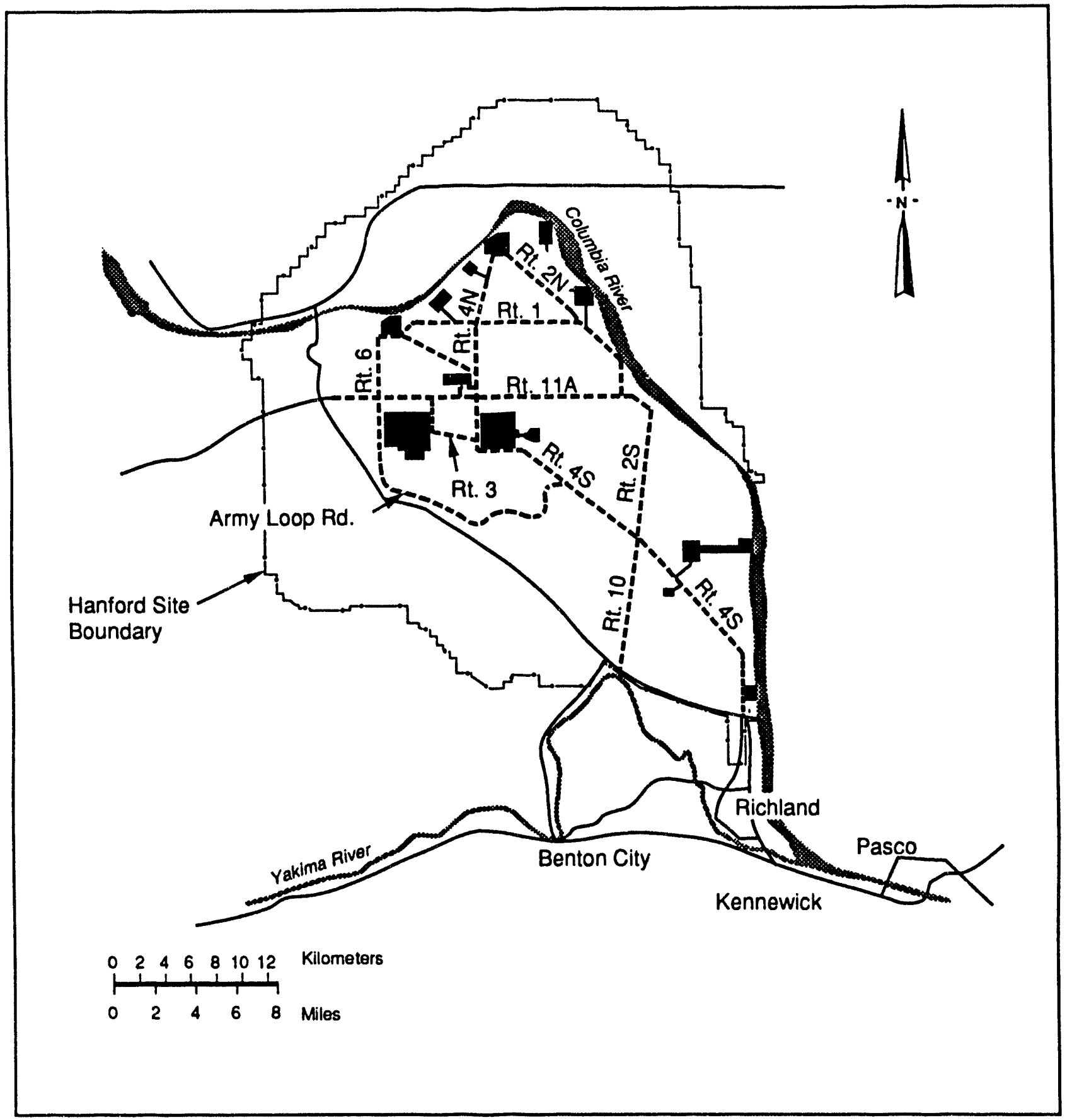

S9108076.1 
WHC-SP-0098-5

Rev. 0

Issue Date: 12/15/93

200 East Area Roads and Railways.
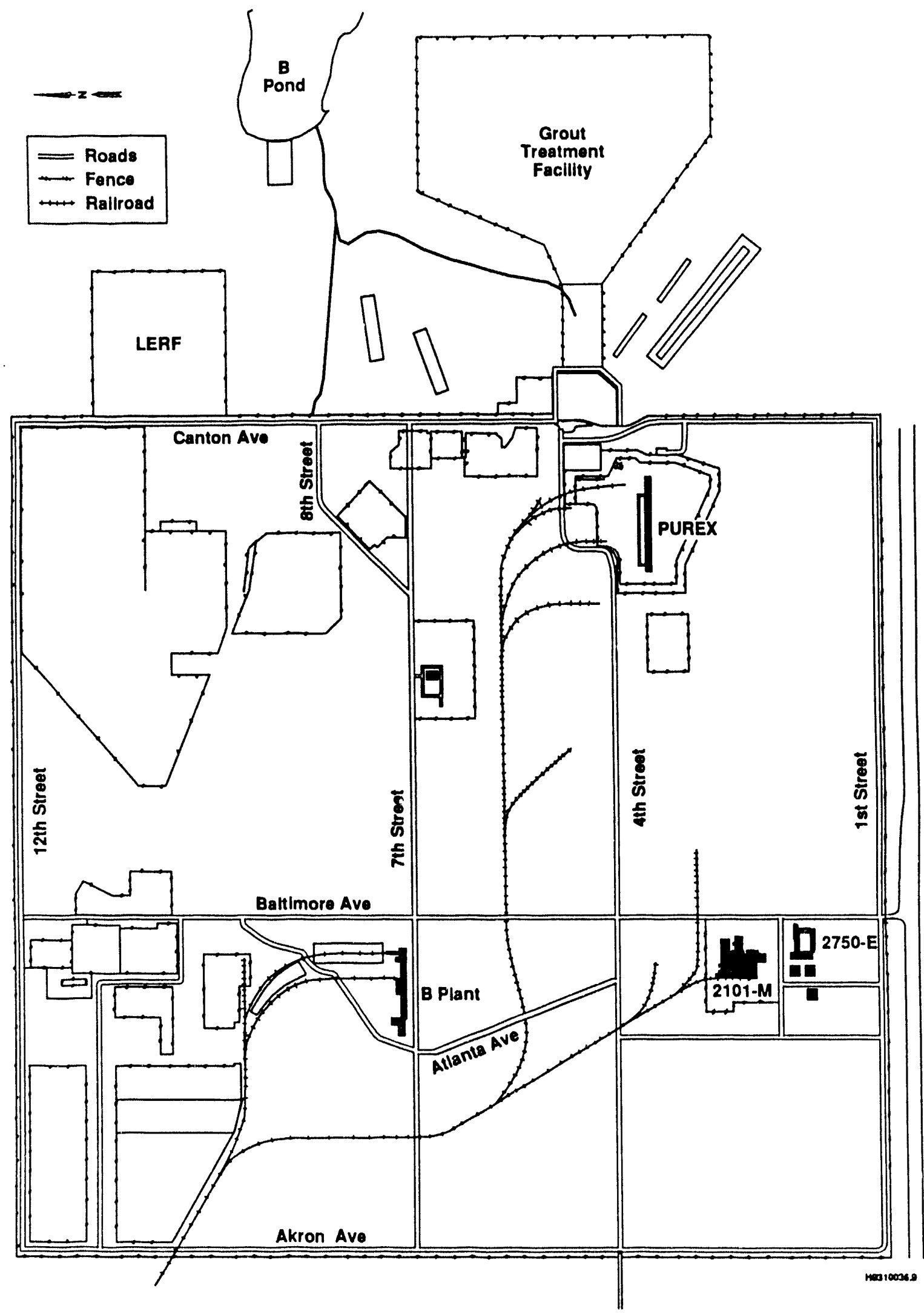
WHC-SP-0098-5

Rev. 0

Issue Date: $12 / 15 / 93$

200 West Area Roads and Railways.

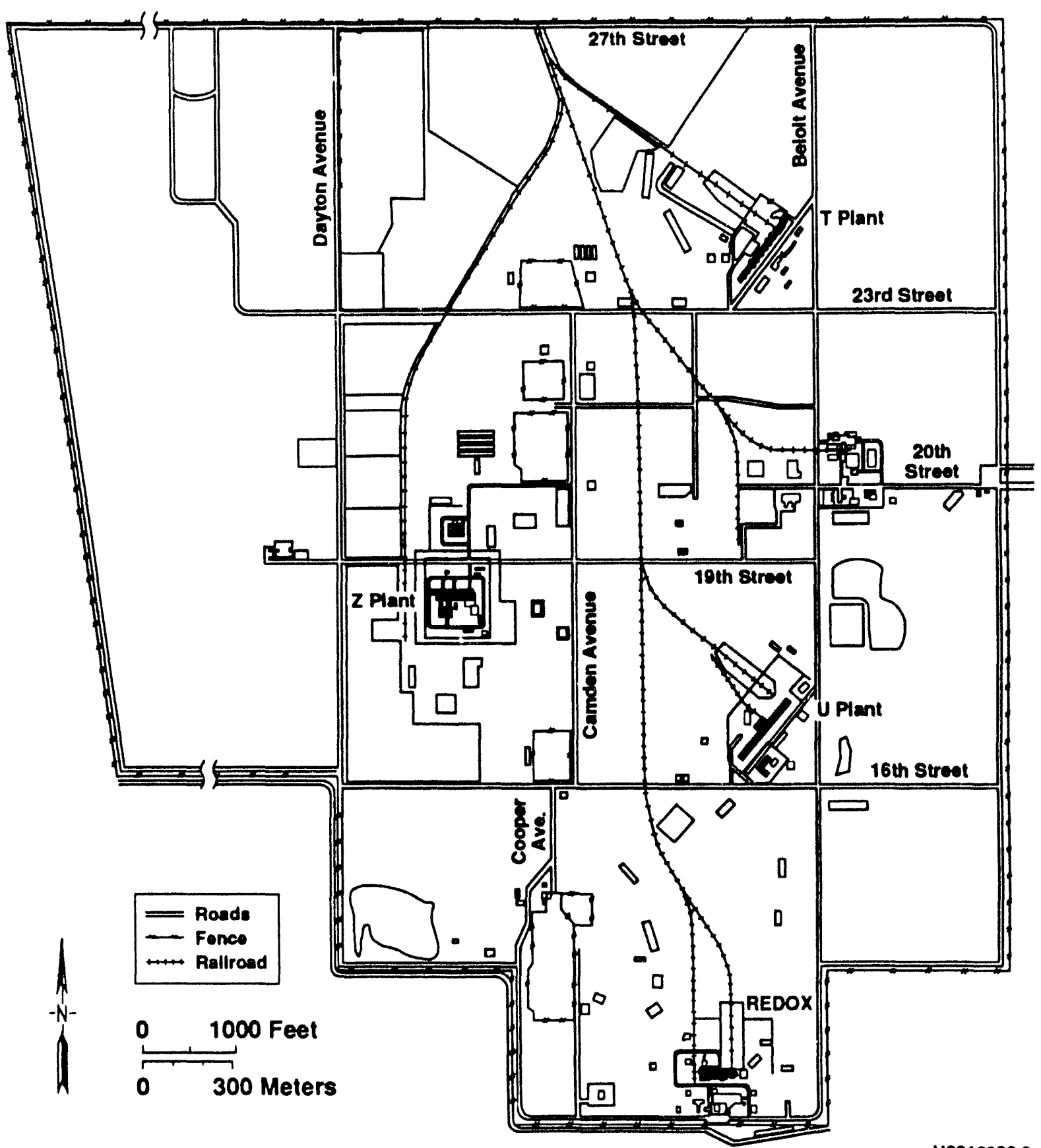

H9310036.8 
WHC-SP-0098-5

Rev. 0

Issue Date: 12/15/93

Site Railways.

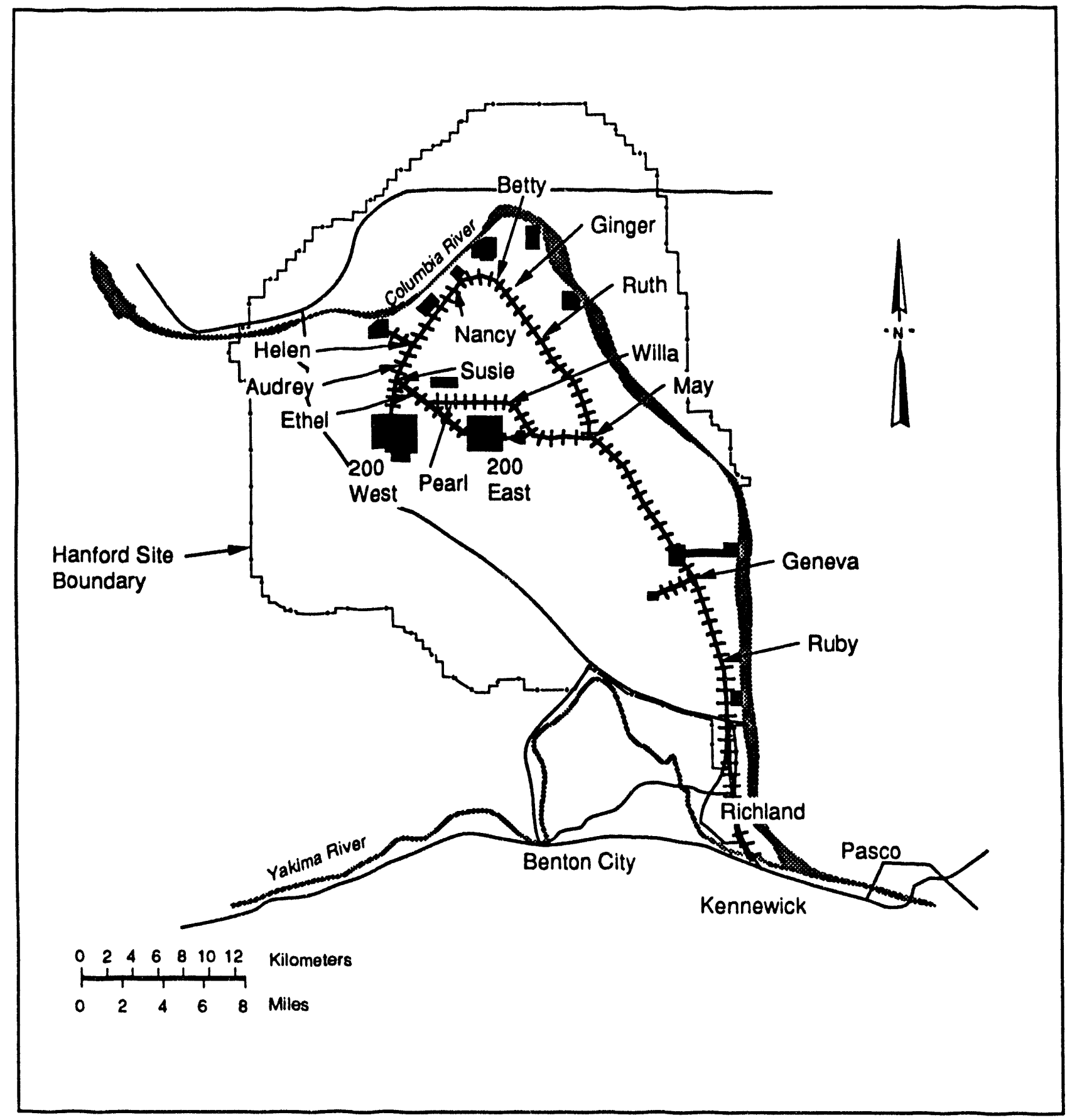

S9108076.2 
WHC-SP-0098-5

Rev. 0

Issue Date: 12/15/93

100-N Area Ambient Air Sample Location.

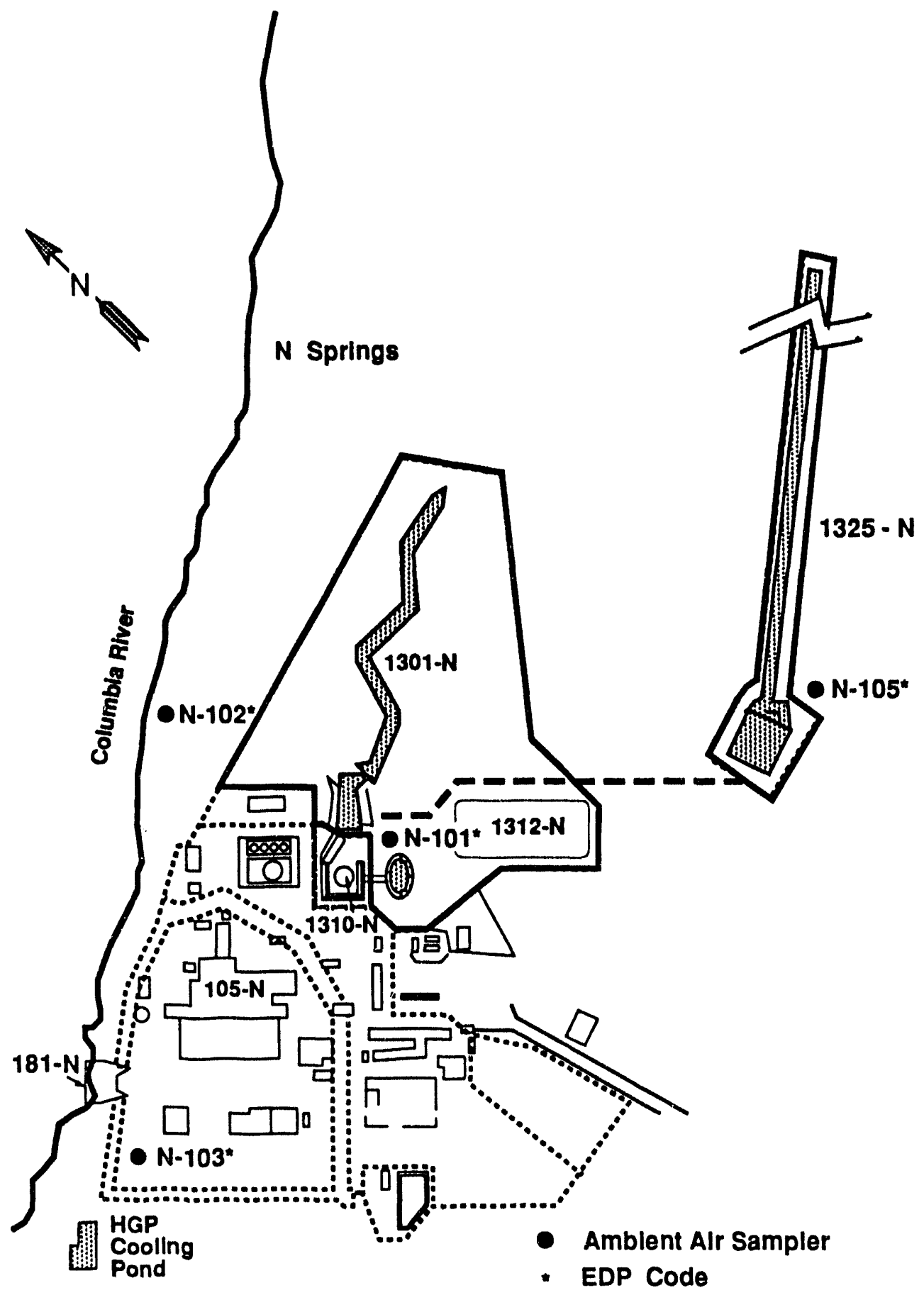

29204047.1 
WHC-SP-0098-5

Rev. 0

Issue Date: $12 / 15 / 93$

100-K Area Ambient Air Sample Location.

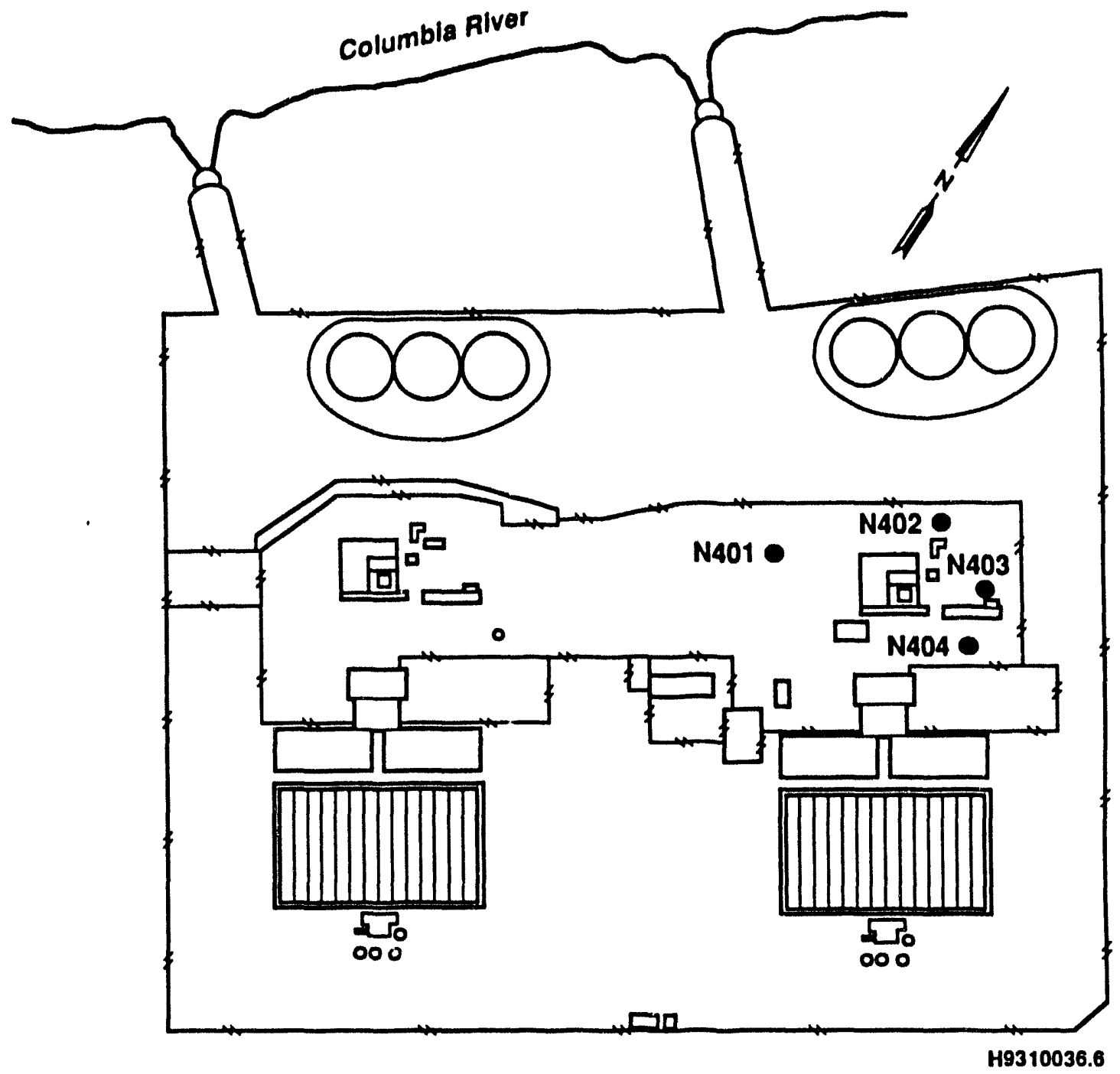


WHC-SP-0098-5

Rev. 0

Issue Date: 12/15/93

200 East Area Air Sample Locations.

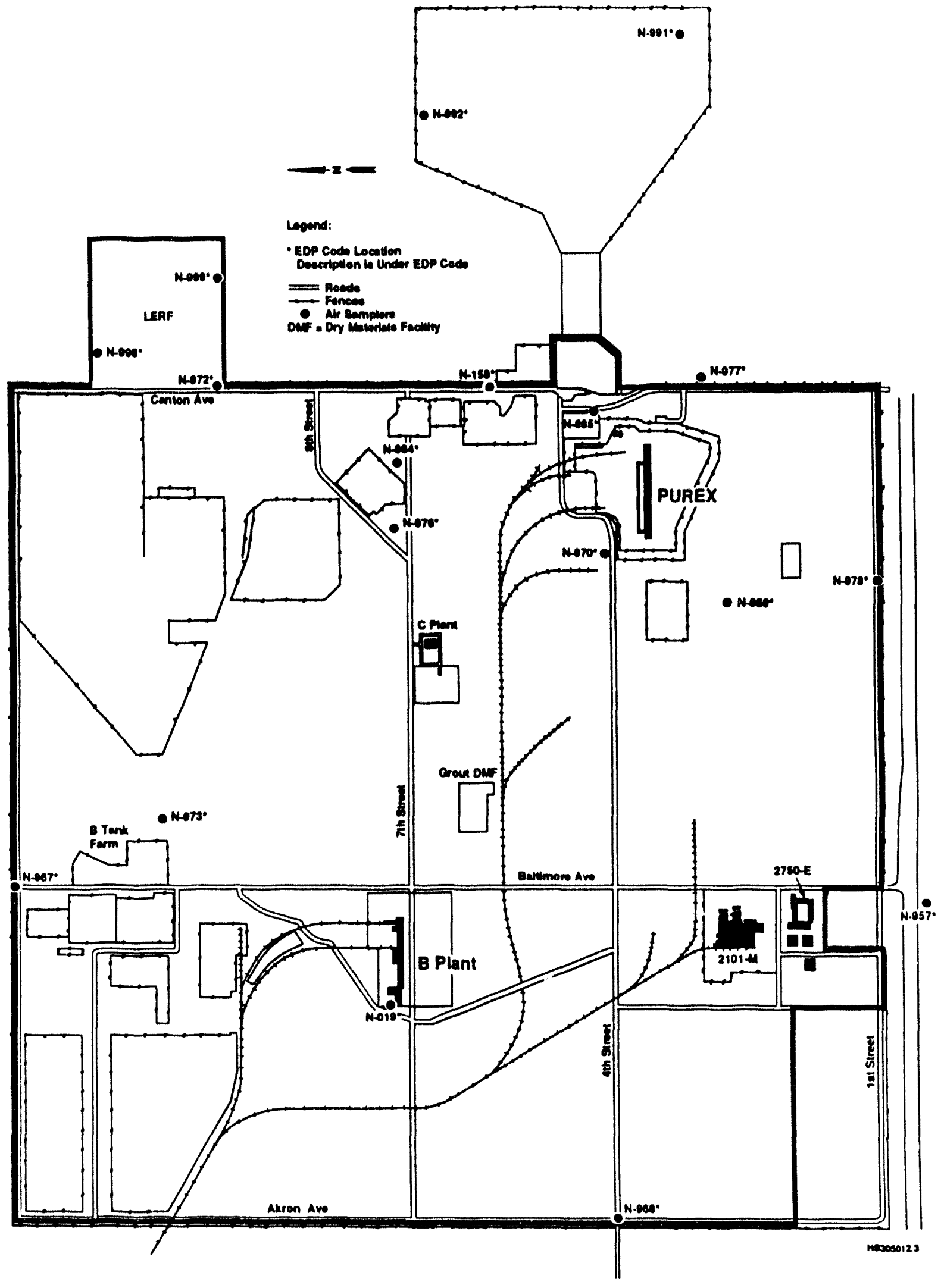


WHC-SP-0098-5

Rev. 0

Issue Date: 12/15/93

200 West Area Air Sample Locations.

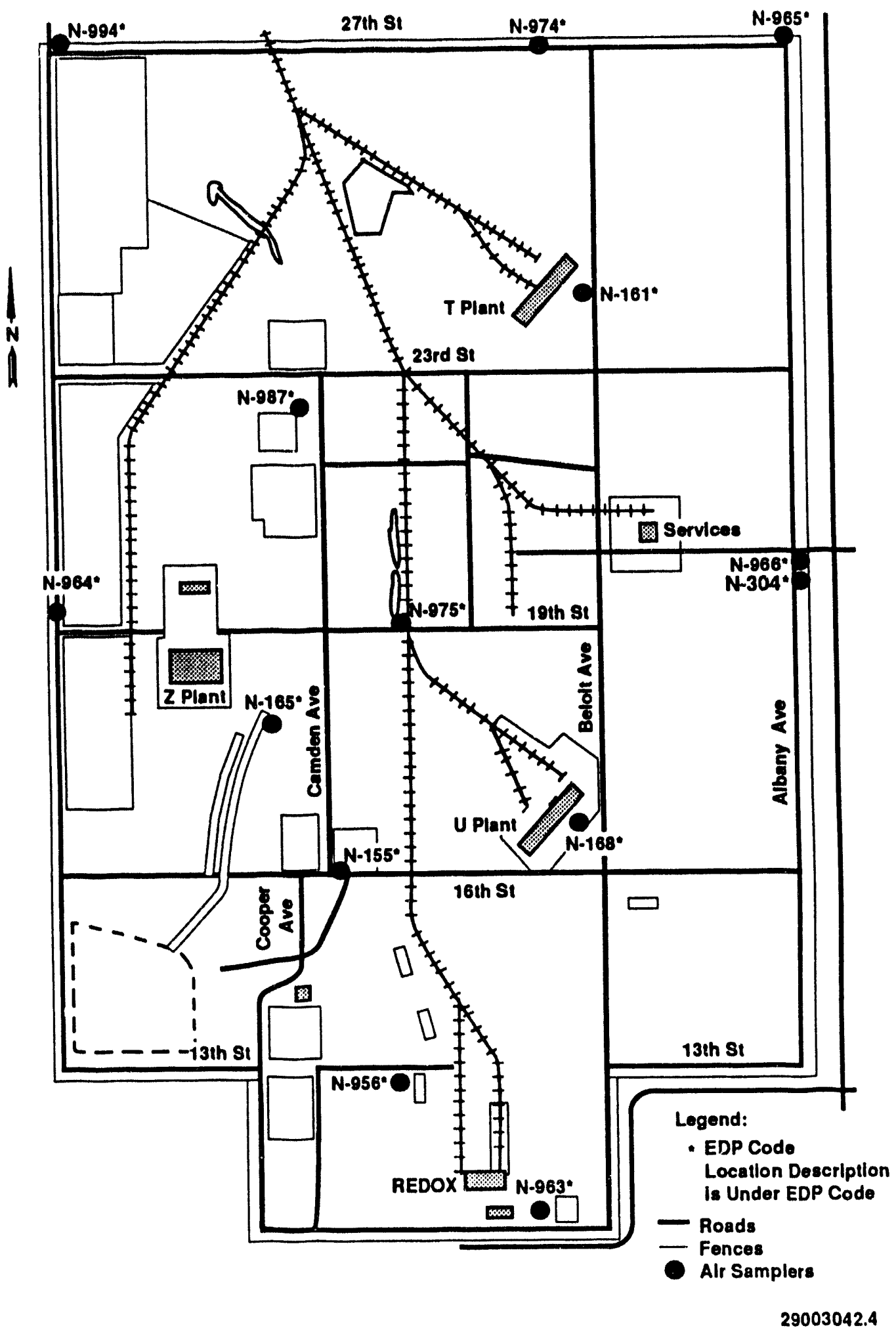


WHC-SP-0098-5

Rev. 0

Issue Date: 12/15/93

200 East/West Areas Vent Station Air Sample Locations.

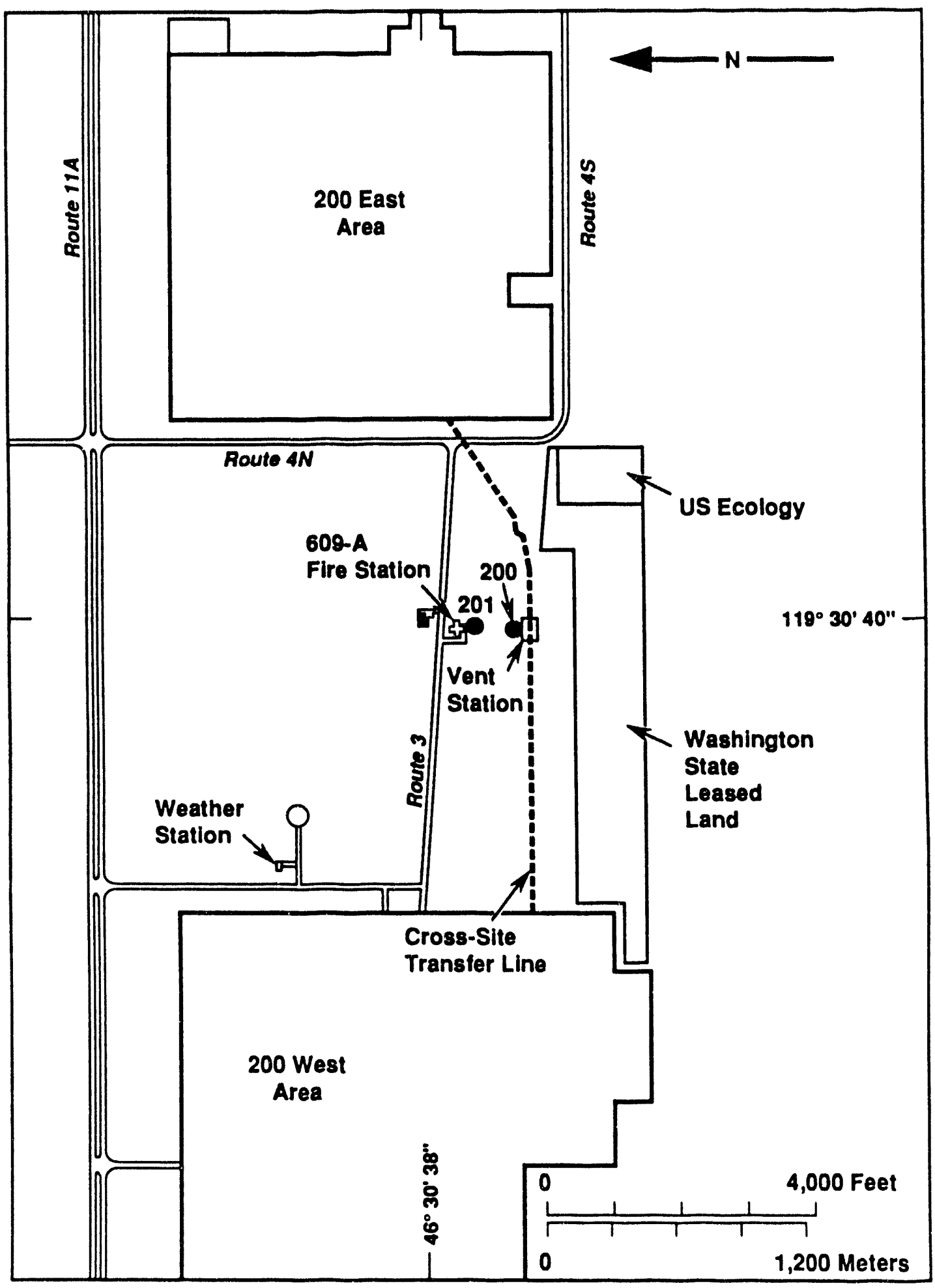

H9306026.1 
WHC-SP-0098-5

Rev. 0

Issue Date: 12/15/93

600 Area Air Sample Location.



29003042.7 
WHC-SP-0098-5

Rev. 0

Issue Date: 12/15/93

100-N Area TLD Locations.

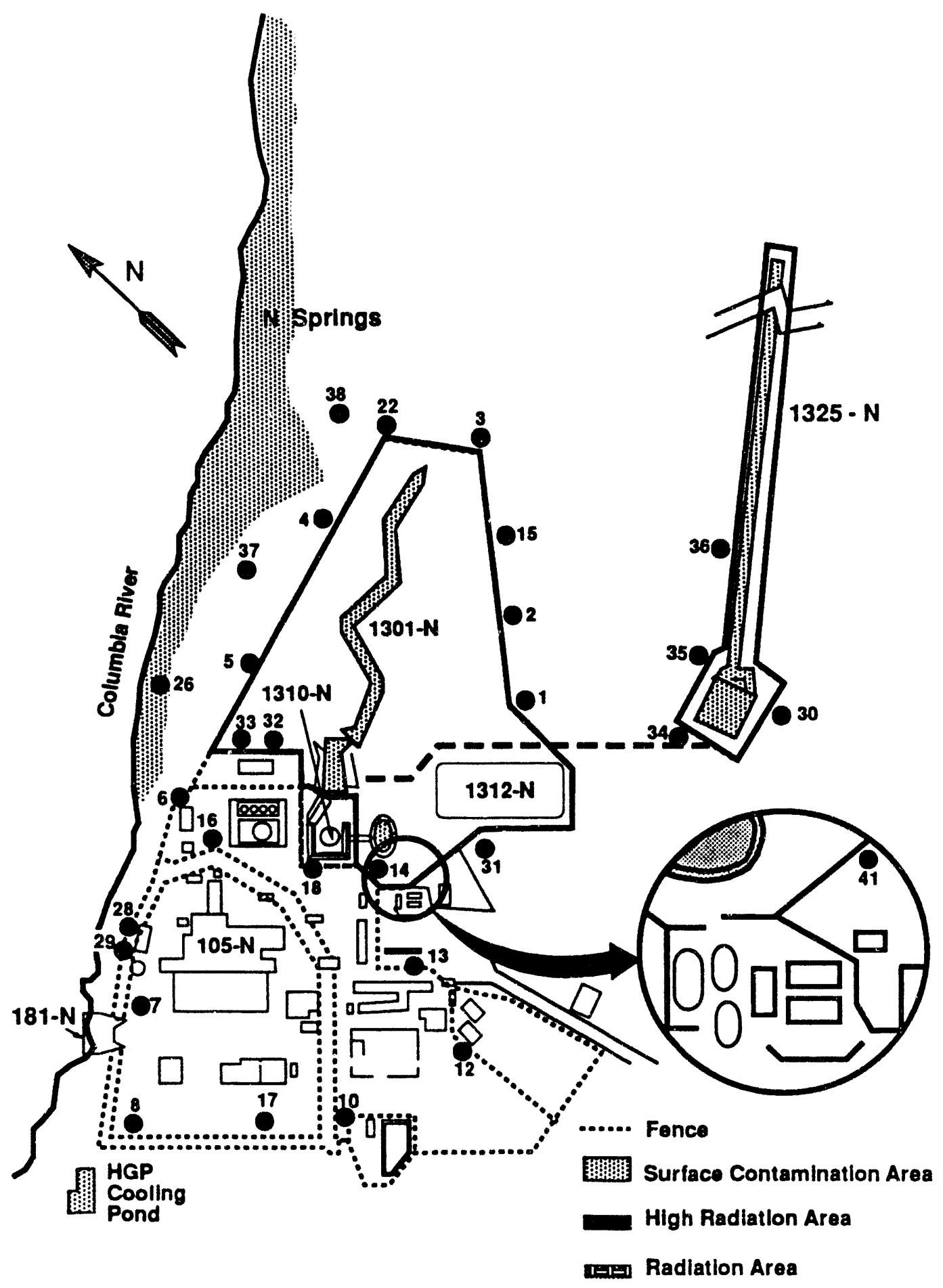

$29202060.1 \mathrm{~A}$ 
WHC-SP-0098-5

Rev. 0

Issue Date: 12/15/93

N Springs Dose Rate Locations.

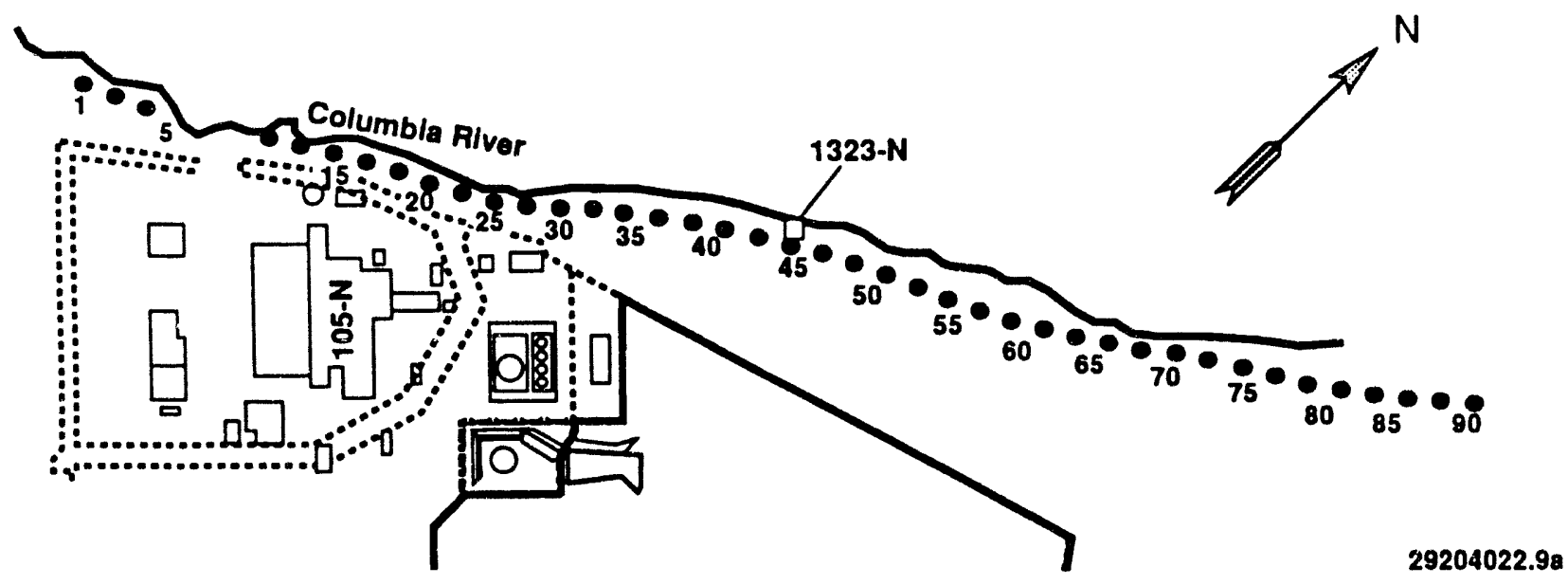


WHC-SP-0098-5

Rev. 0

Issue Date: 12/15/93

1301-N Building Dose Rate Locations.

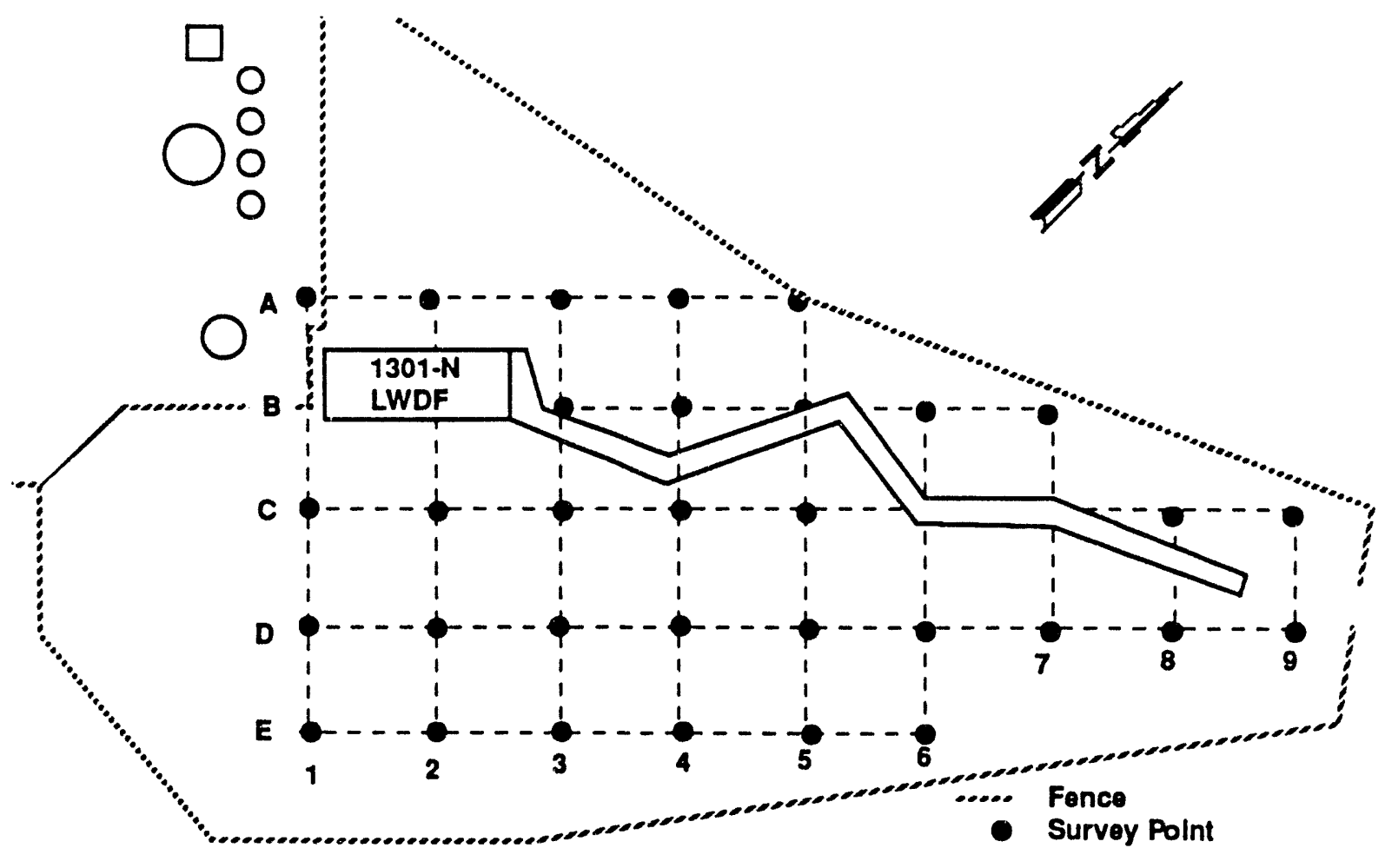

29204022.10 
WHC-SP-0098-5

Rev. 0

Issue Date: 12/15/93

1325-N Building Dose Rate Locations.
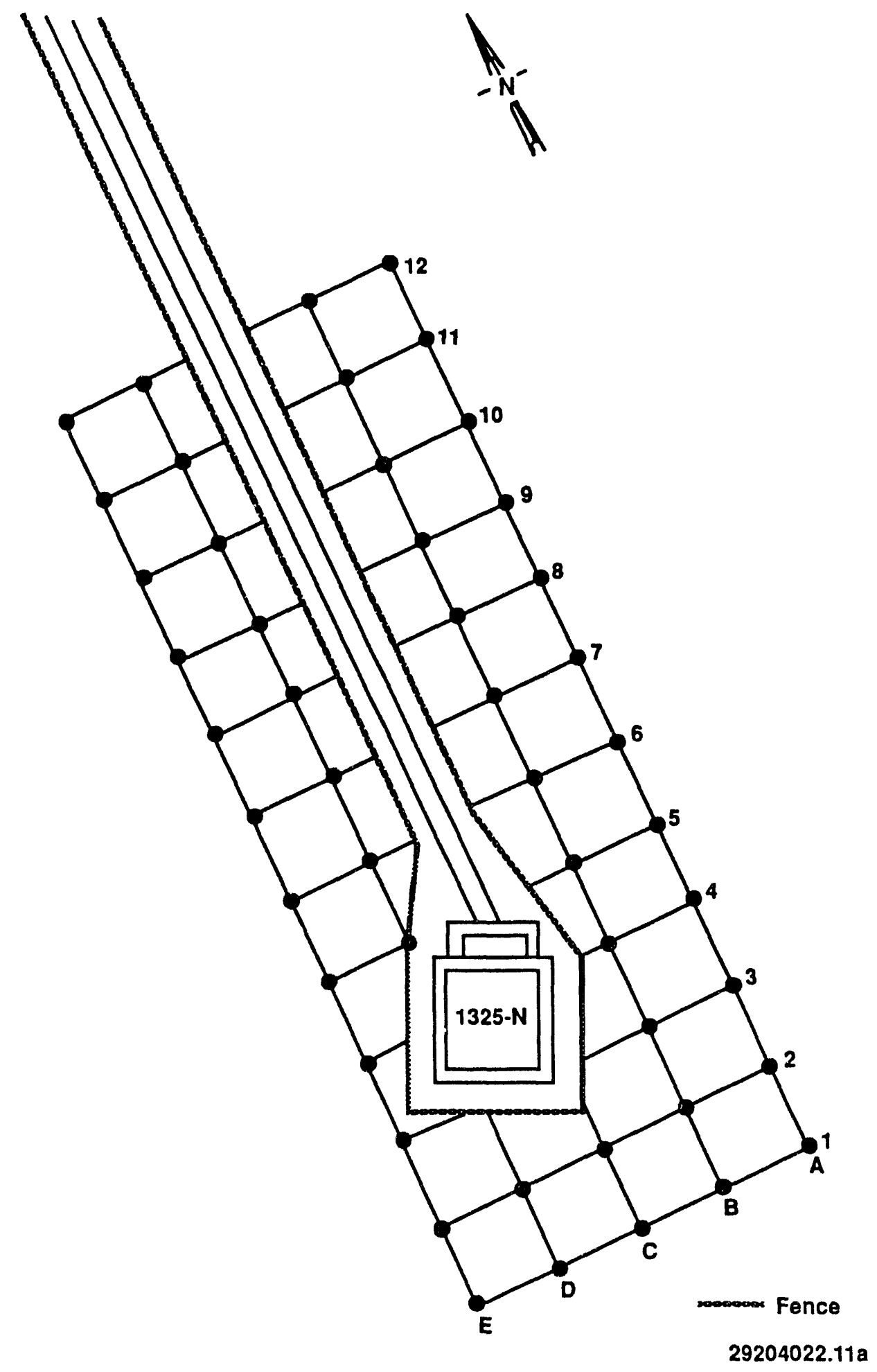
WHC-SP-0098-5

Rev. 0

Issue Date: $12 / 15 / 93$

100-K Area TLD Locations.

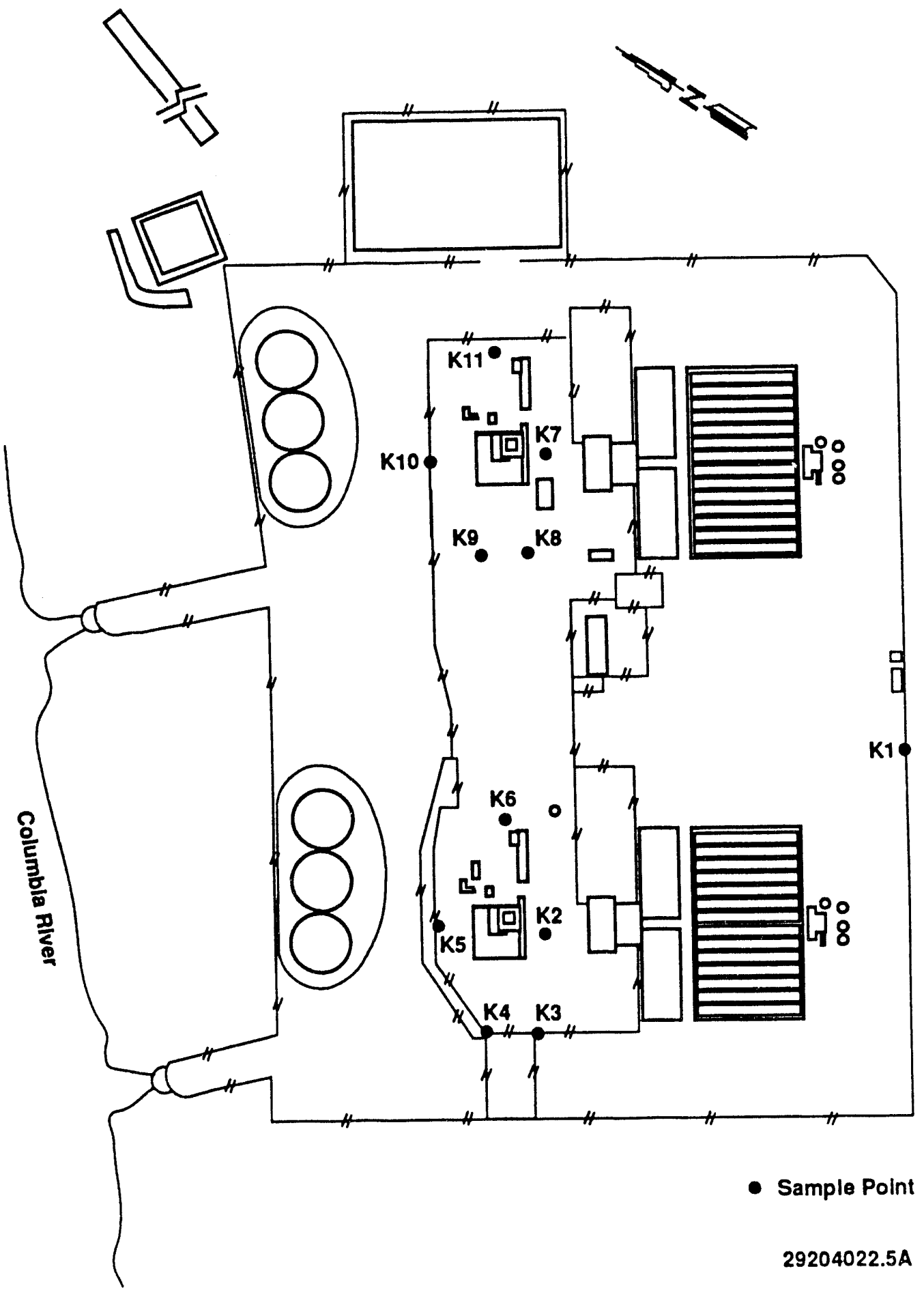


WHC-SP-0098-5

Rev. 0

Issue Date: 12/15/93

200 East Area TLD Locations.

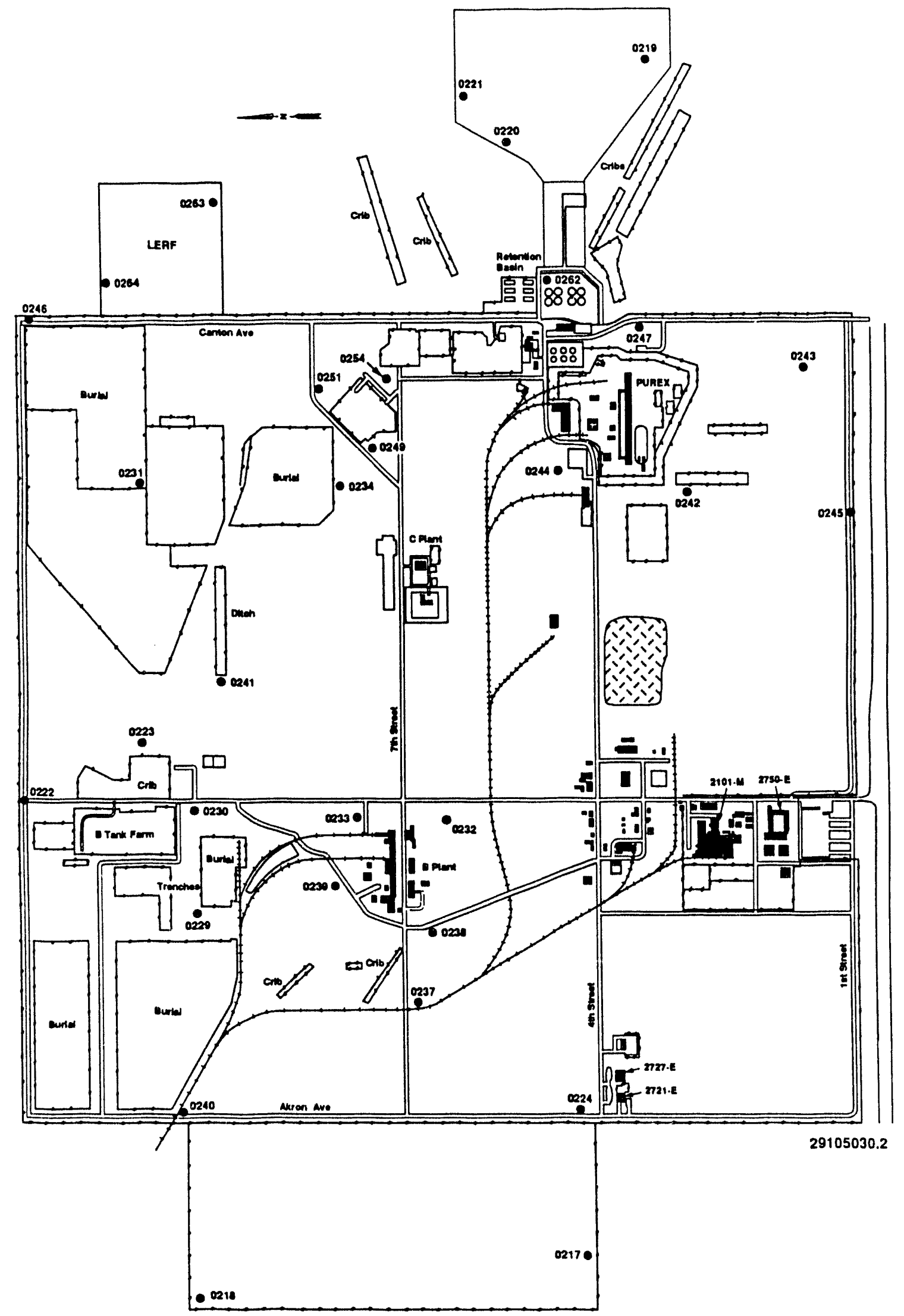


WHC-SP-0098-5

Rev. 0

Issue Date: 12/15/93

200 East Area, "A" Tank Farm TLD Locations.

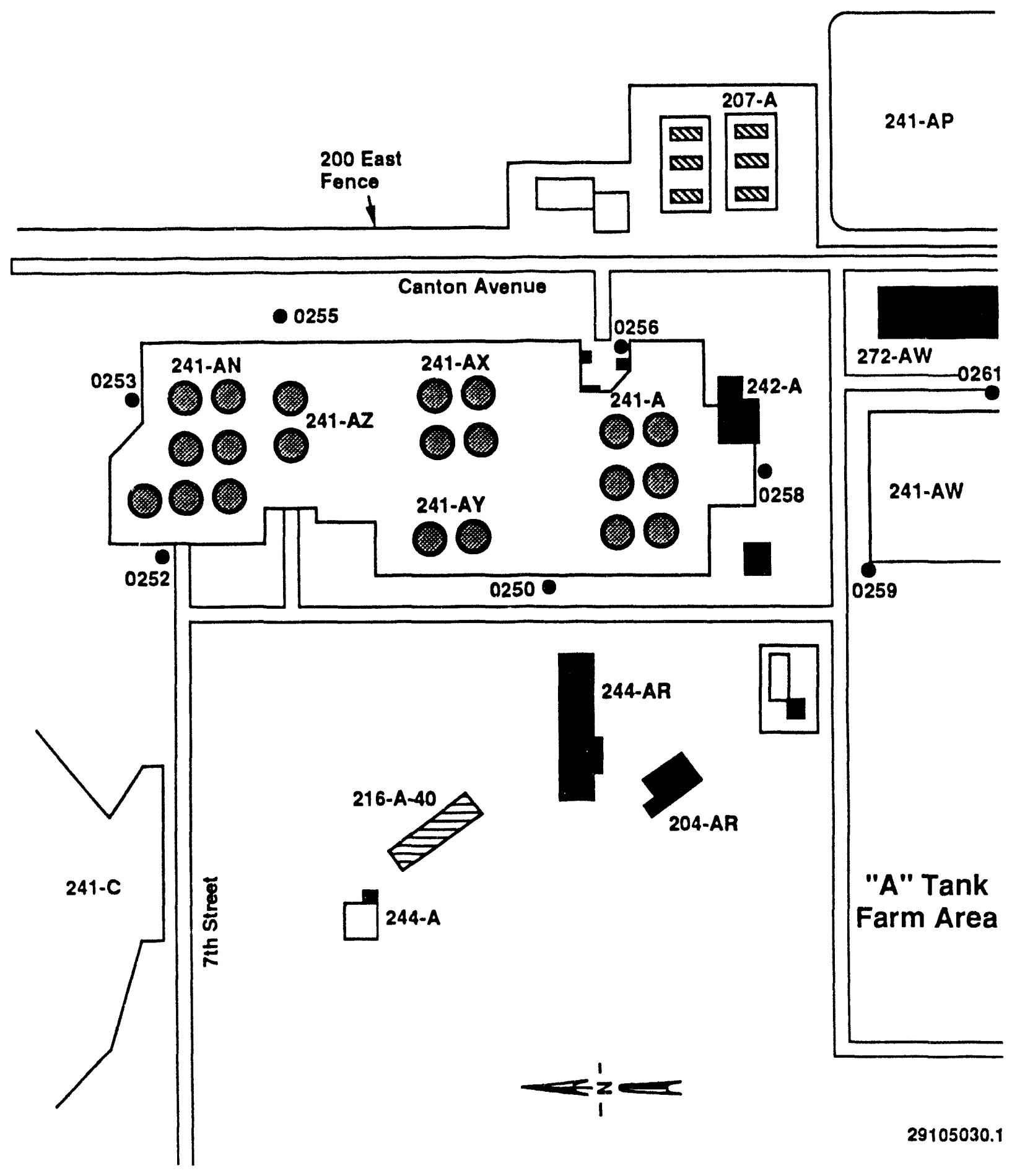


WHC-SP-0098-5

Rev. 0

Issue Date: $12 / 15 / 93$

200 West Area TLD Locations.

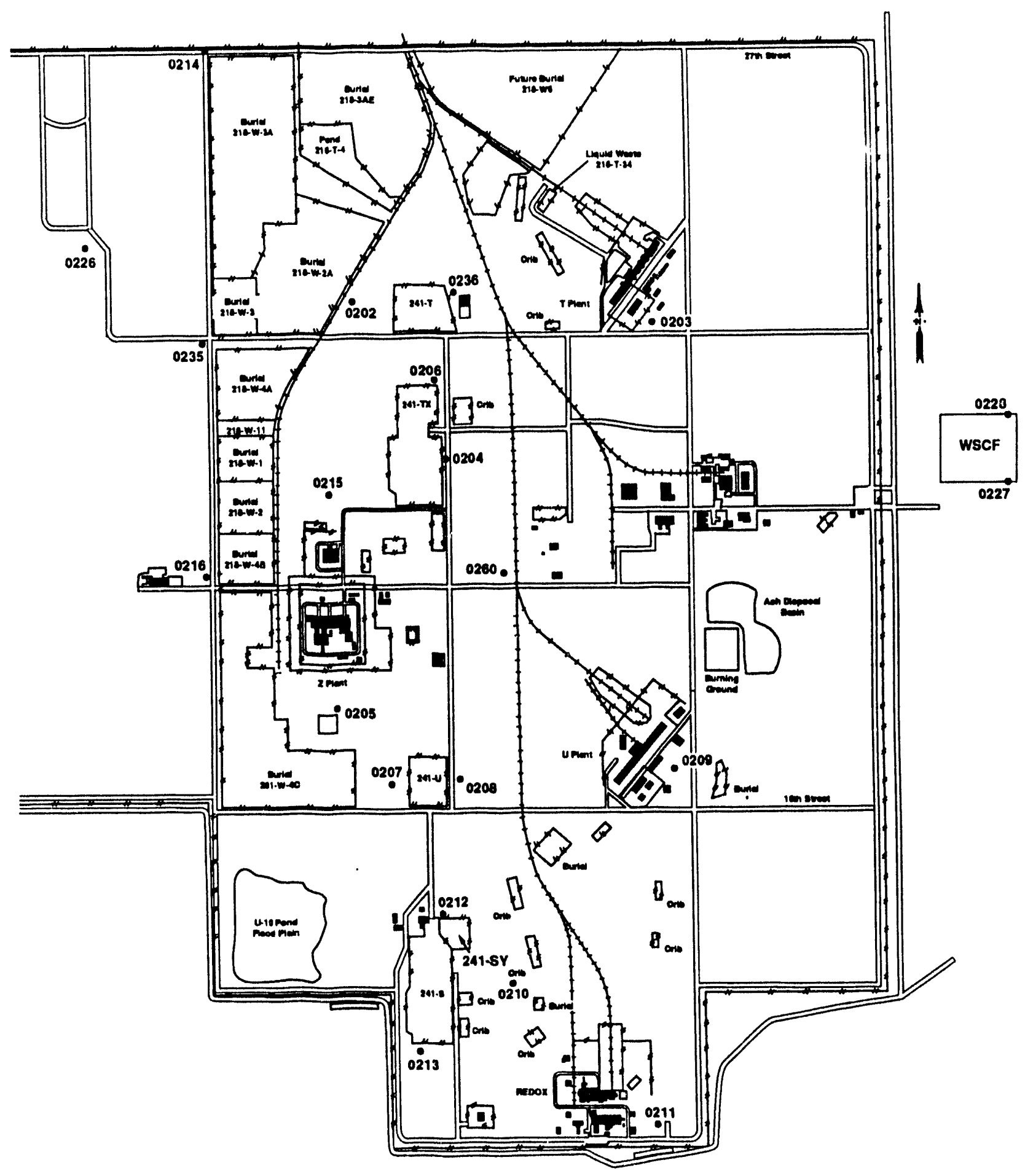

29105030.3 
WHC-SP-0098-5

Rev. 0

Issue Date: 12/15/93

300 Area TLD Locations.

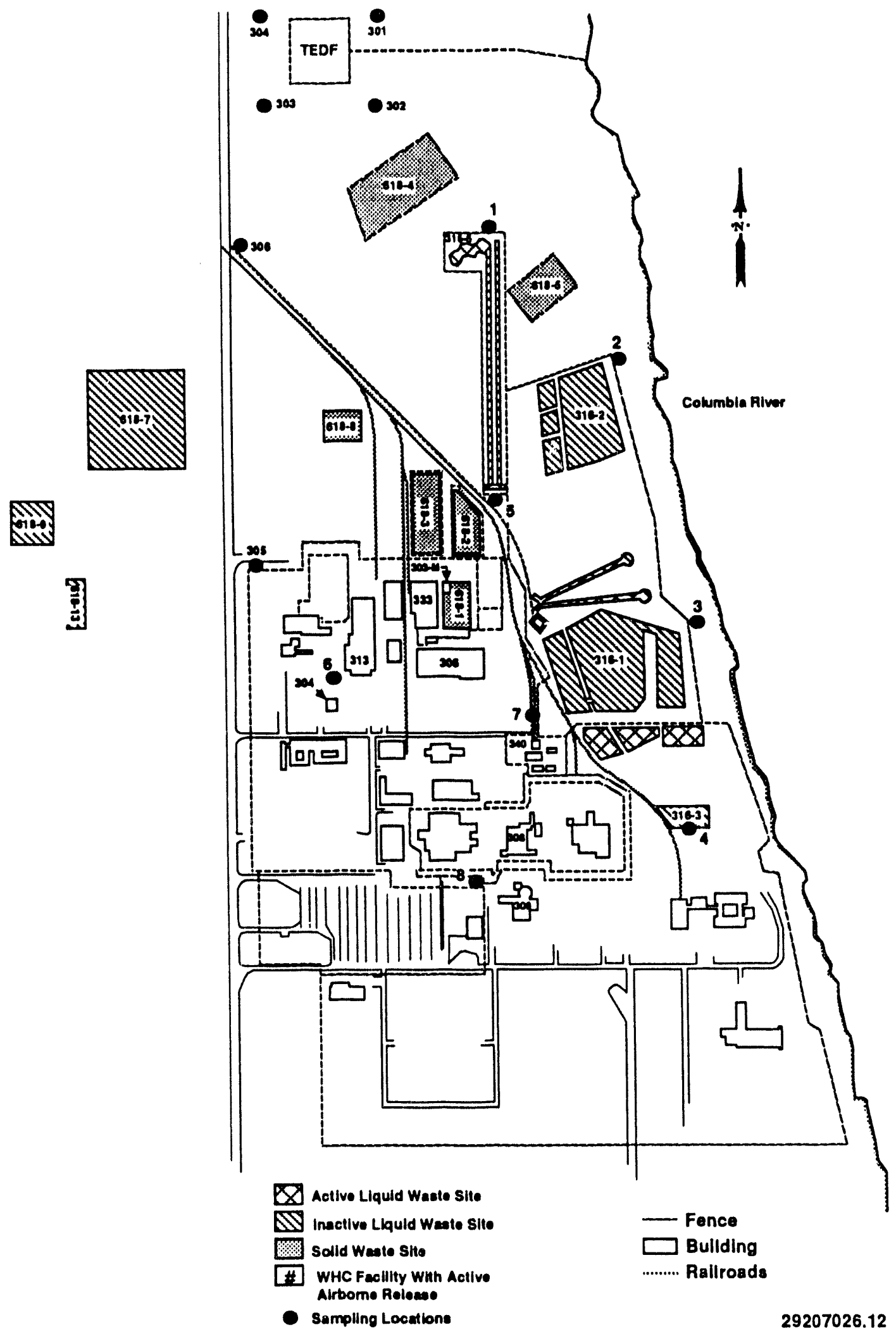


WHC-SP-0098-5

Rev. 0

Issue Date: 12/15/93

400 Area TLD Locations.
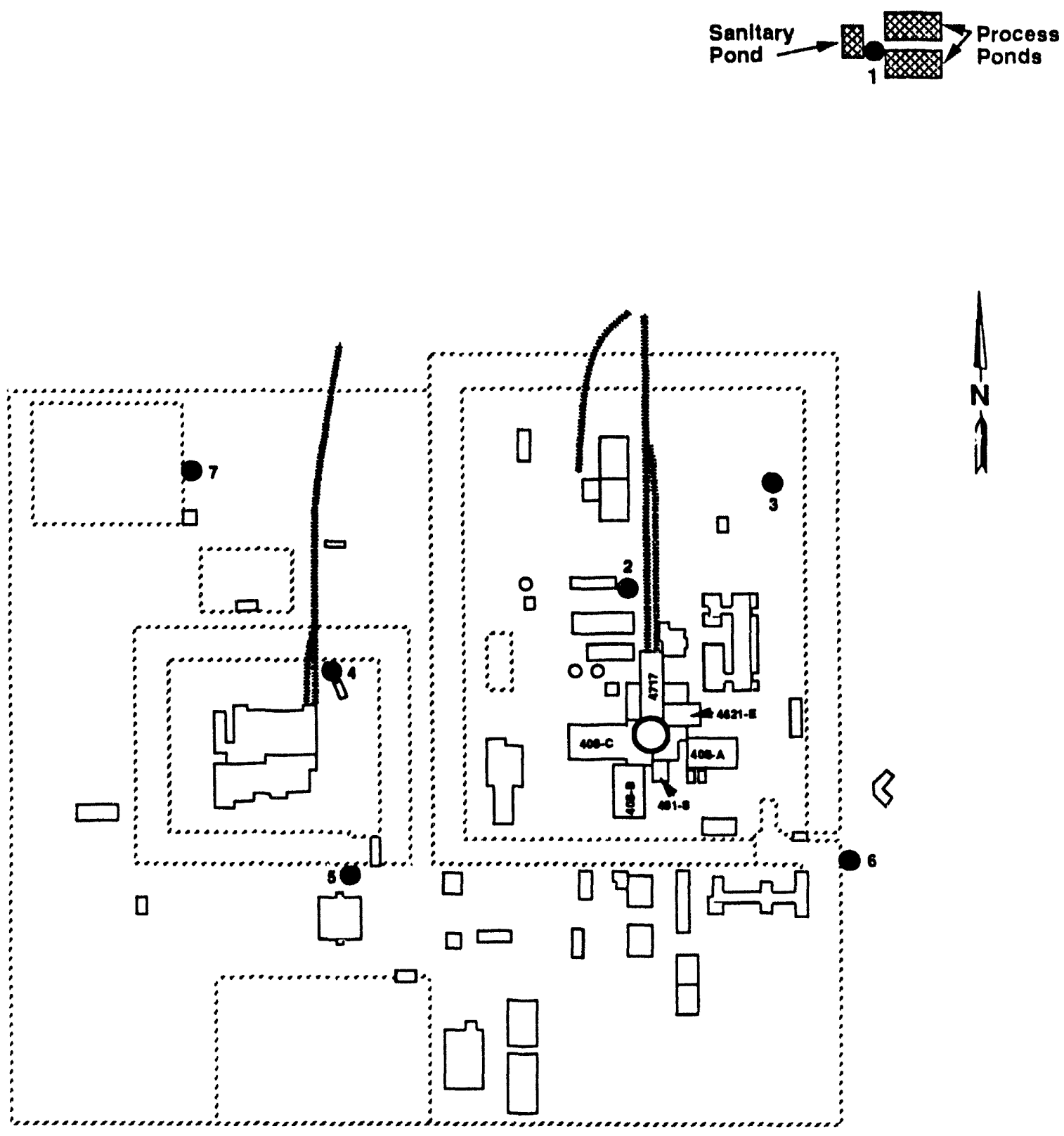

Active Liquild Waste Site

$\square$ Facility with Active Alrborne Releases

- Sample Locations

Fence

39003105.2 
WHC-SP-0098-5

Rev. 0

Issue Date: 12/15/93

100-N Area Soll and Vegetation Sample Locations.

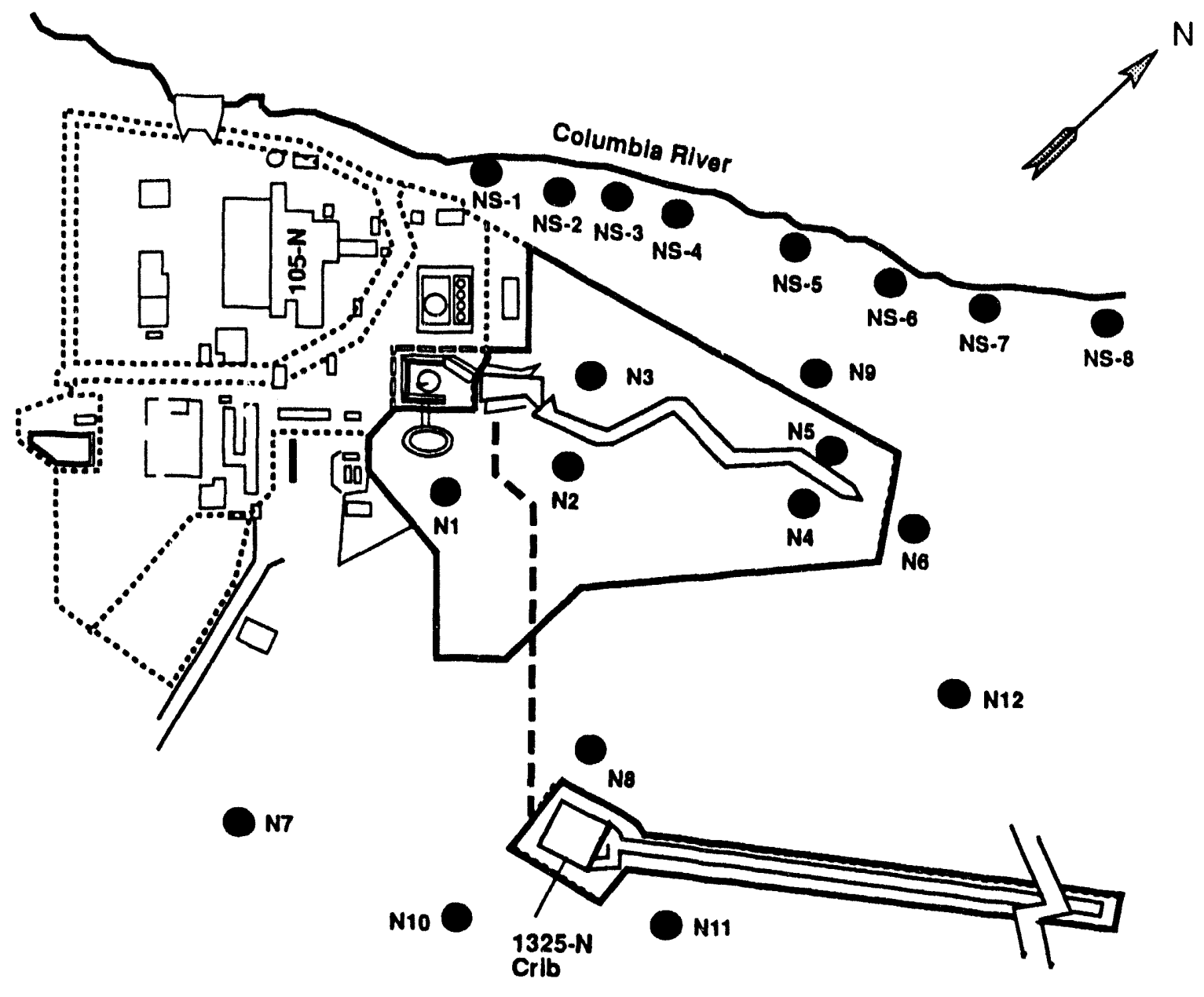

Sample Point

29204022.24 
WHC-SP-0098-5

Rev. 0

Issue Date: 12/15/93

200 East Area Soil and Vegetation Sample Locations.

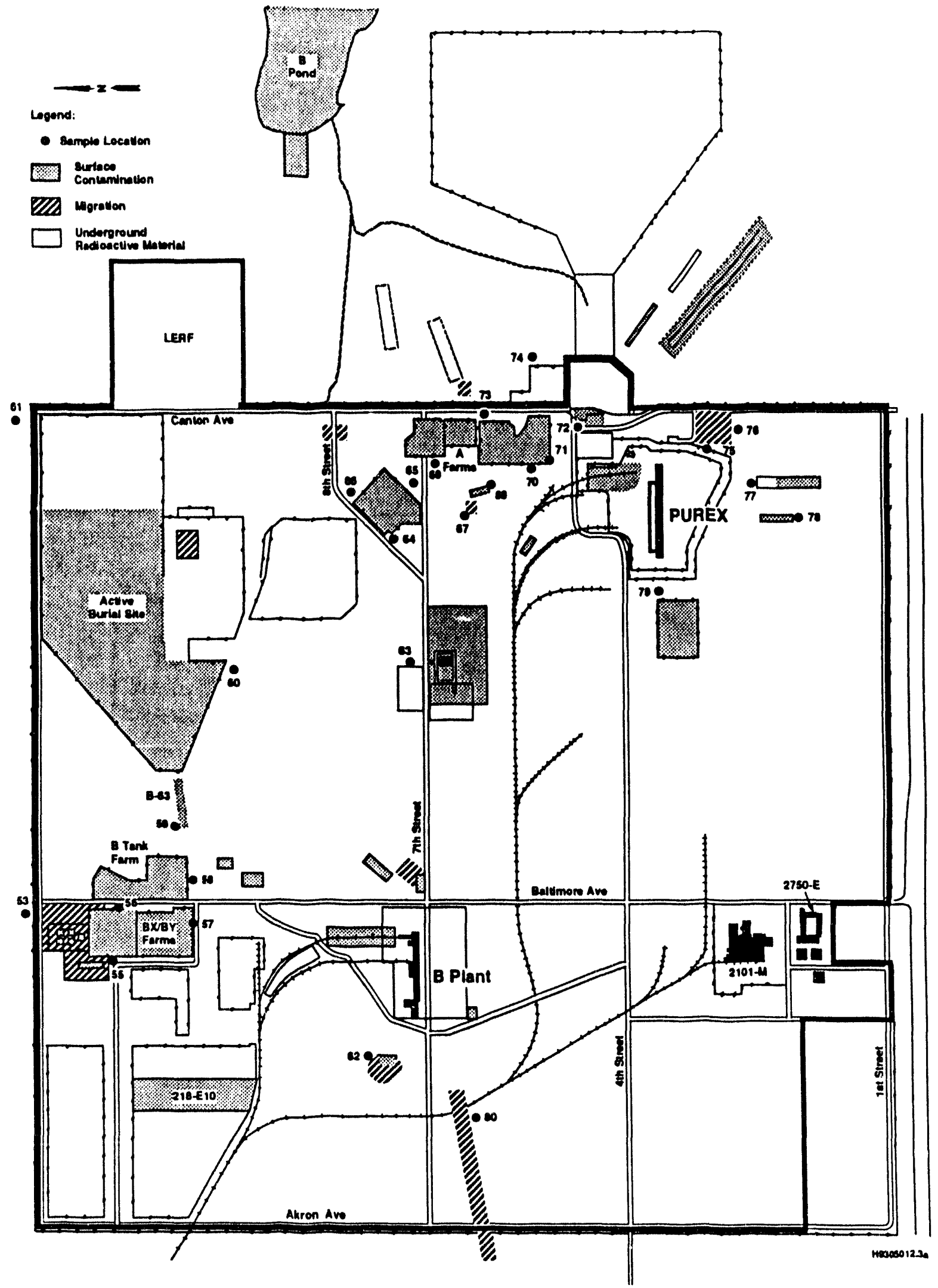


WHC-SP-0098-5

Rev. 0

Issue Date: 12/15/93

200 West Area Soil and Vegetation Sample Locations.

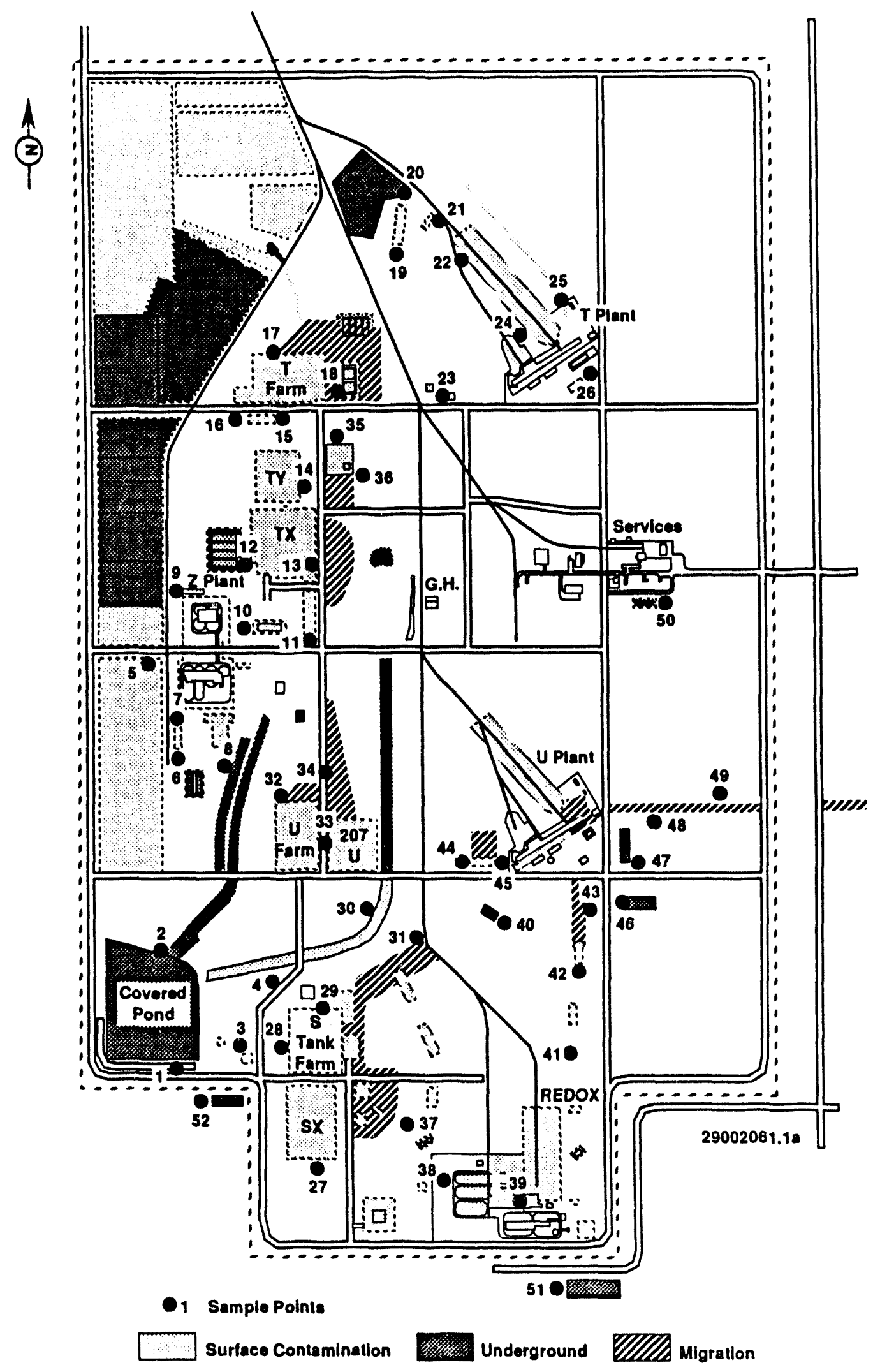


WHC-SP-0098-5

Rev. 0

Issue Date: $12 / 15 / 93$

600 Area Soil and Vegetation Sample Locations.

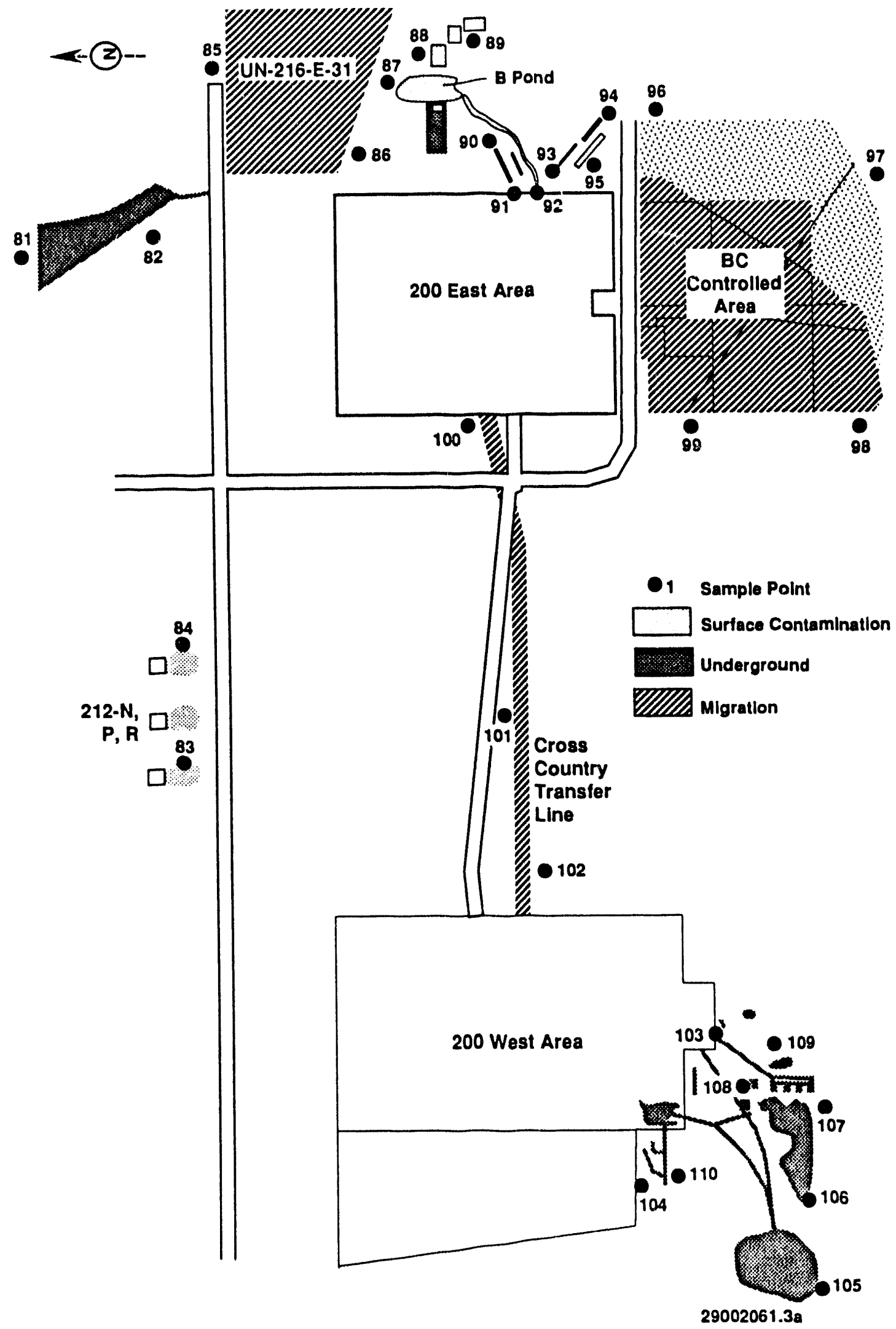


WHC-SP-0098-5

Rev. 0

Issue Date: 12/15/93

300 Area Soil and Vegetation Sample Locations.

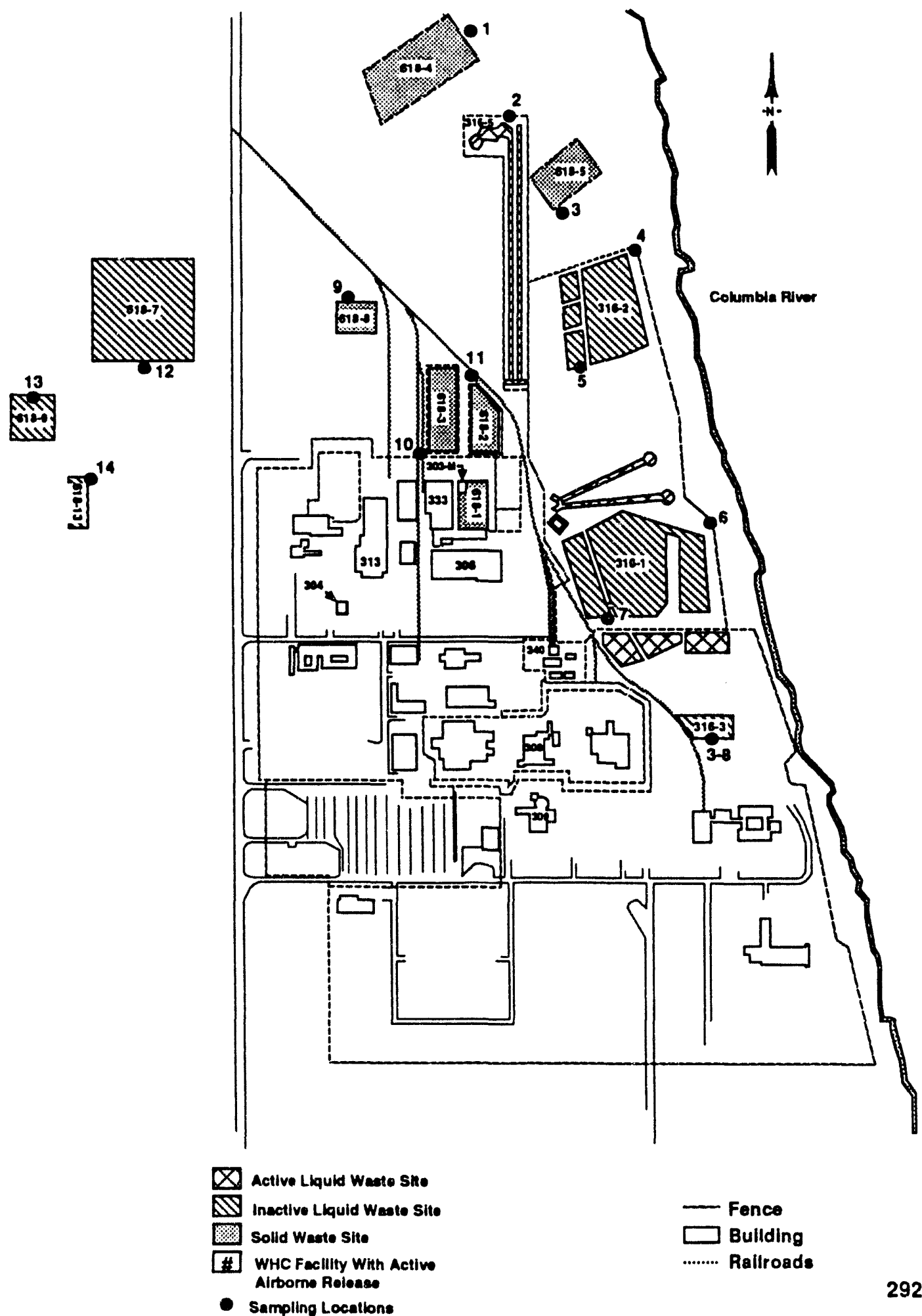

29207026.2 
WHC-SP-0098-5

Rev. 0

Issue Date: $12 / 15 / 93$

400 Area Soil and Vegetation Sample Locations.
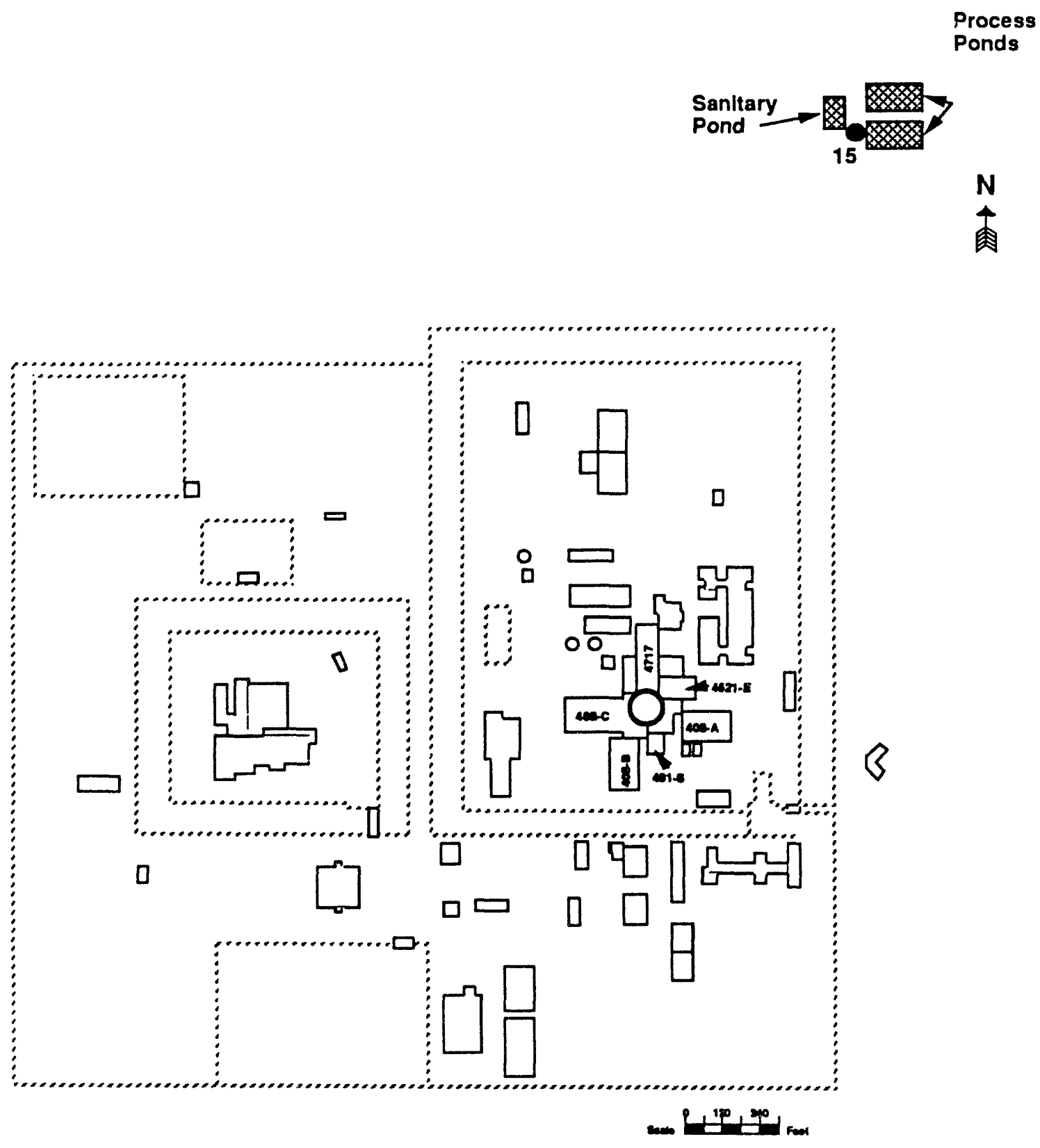

Active Liquid Waste Site

\# Facility with Active Airborne Releases

- Sampling Locations

Fence

29207026.4 
WHC-SP-0098-5

Rev. 0

Issue Date: 12/15/93

200 East Area Water, Sediment, and Aquatic

Vegetation Sample Locations.

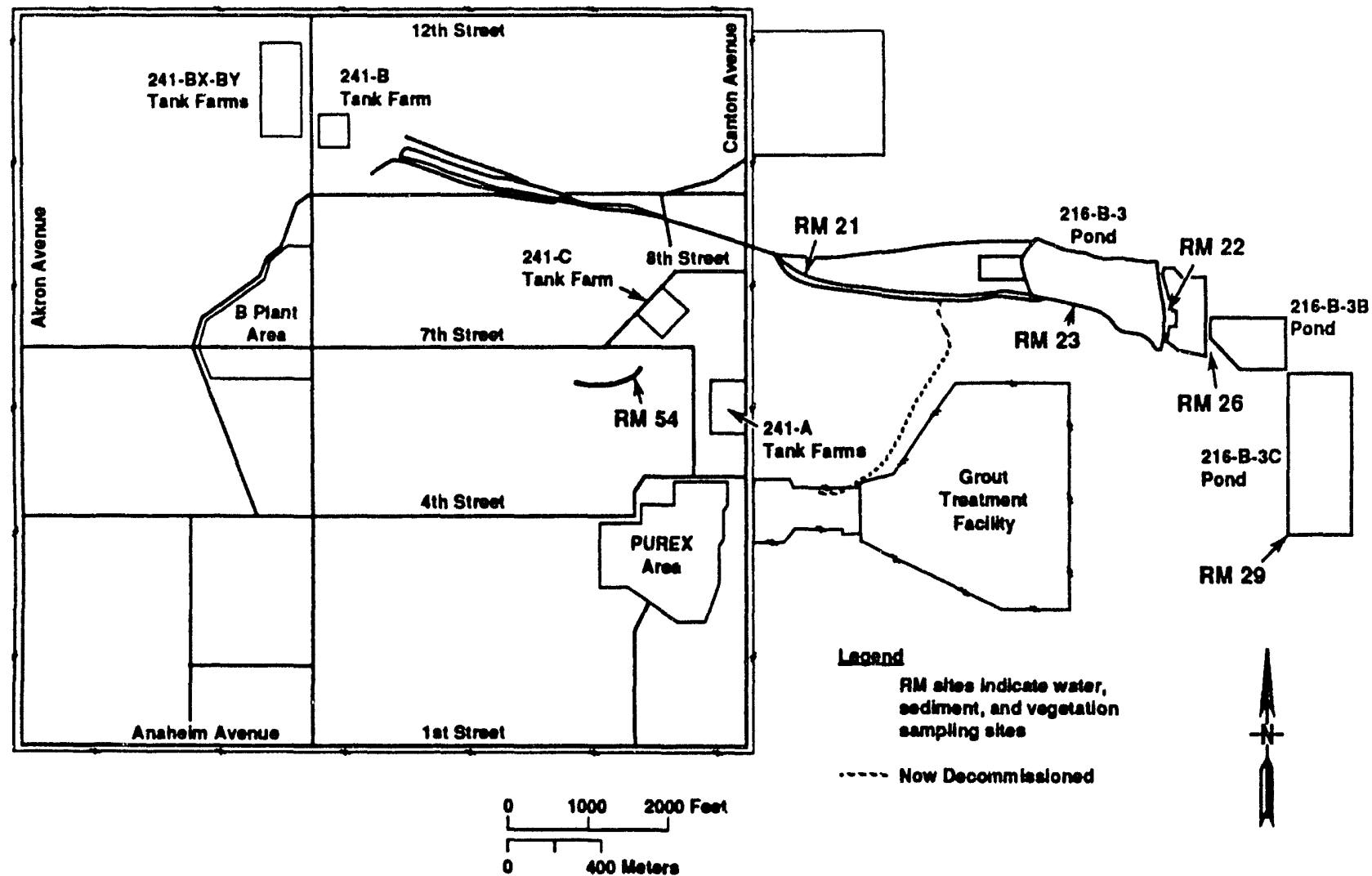

Ho310036.7 
WHC-SP-0098-5

Rev. 0

Issue Date: 12/15/93

200 West Area Water, Sediment, and Aquatic

Vegetation Sample Locations.

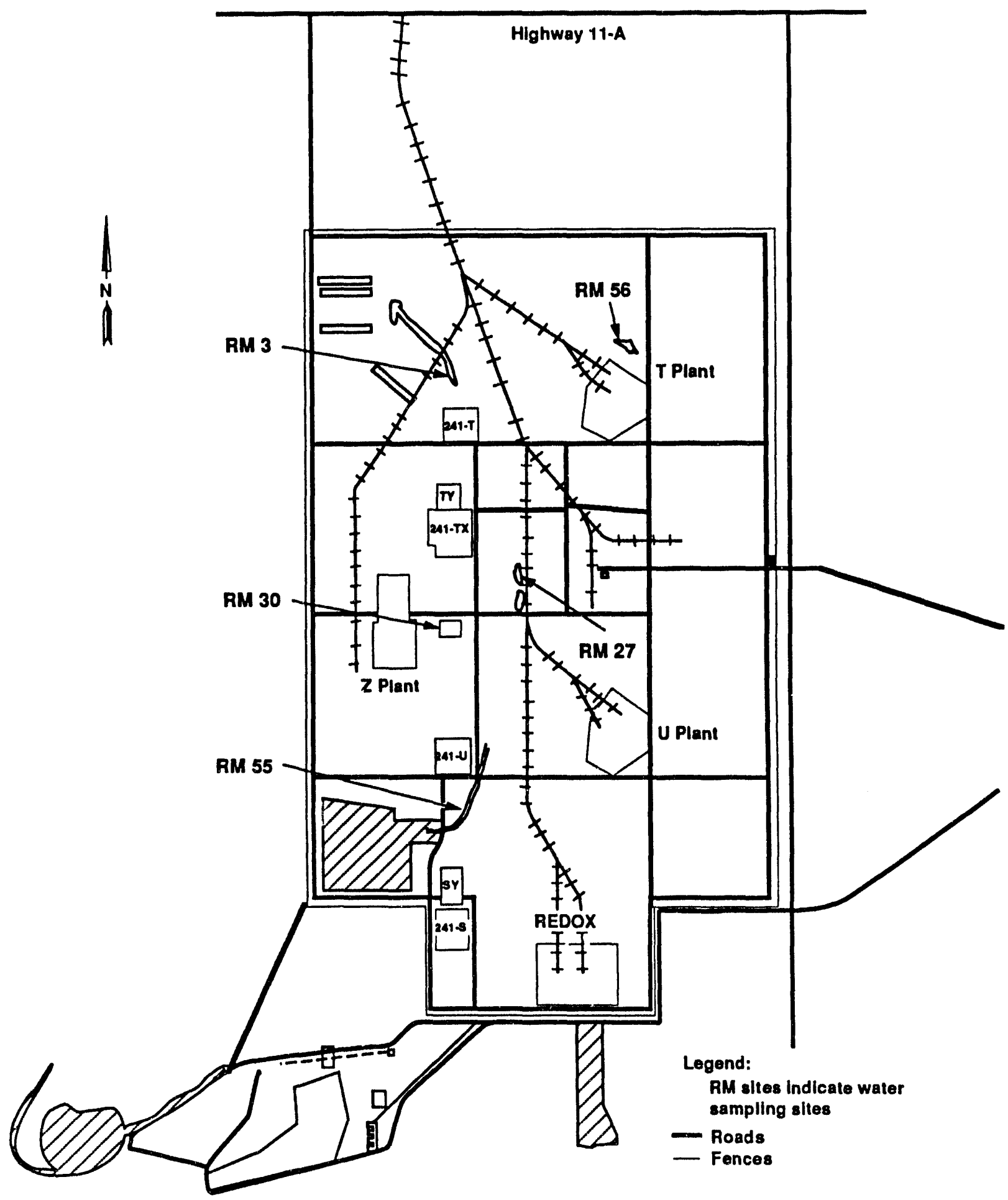

$29003042.2 a$ 
WHC-SP-0098-5

Rev. 0

Issue Date: 12/15/93

N Springs Seepage Sample Locations.

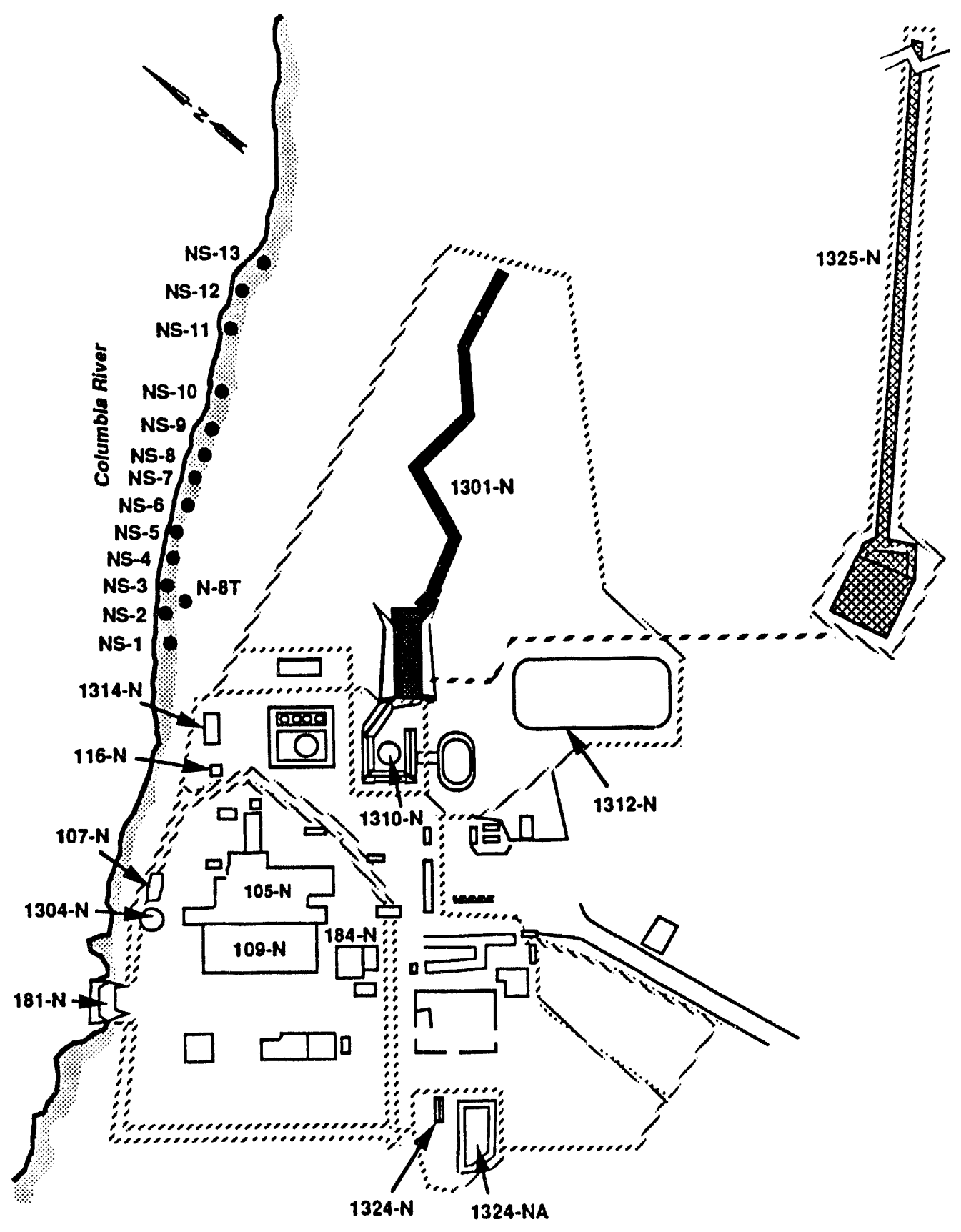

39006103.1 
WHC-SP-0098-5

Rev. 0

Issue Date: 12/15/93

\section{JANUARY}

Inactive Facilities

Semi annual

1. UN-216-W-5

Annual

1. 216-S-10 (In ctive Section)

2. $216-S-17$

3. UN-216-W-23

\section{Active Facilities}

\section{Weekly}

1. Routine water samples

\section{Biweekly}

1. Routine air samples

\section{Bimonthly}

1. Road surveys inside 200 East Area

2. Road surveys inside 200 West Area

\section{Quarterly}

1. Tritium and nitrate water samples

2. Vent station perimeter and surface contamination area (cross site transfer line)

\section{Semiannual}

1. Composite air samples

2. Outside perimeter roads of 200 East Area

3. Outside perimeter roads of 200 West Area

\section{Annual}

1. Highway survey (Route 4 South) 1100 Area to Wye Barricade, to include 300 and 400 Areas access roads and parking lots

2. Posted Underground Radioactive Material (URM) Pipelines in 200 East Area (excluding East/West Transfer Line) 
WHC-SP-0098-5

Rev. 0

Issue Date: 12/15/93

\section{FEBRUARY}

\section{Inactive Facilities}

\section{Semiannual}

1. 100-B/C (URM) Areas (includes 116-B-1, 116-B-2, 116-B-5, 116-B-7, $116-B-8,116-B-13,116-B-14,116-C-1,116-C-2 A, 116-C-4,118-B-2$, $118-B-3,118-B-4,118-B-5,118-B-6,118-B-7,116-C-5,116-B-11)$

2. 105-B/C Exclusion Areas (includes 116-B-3, 116-B-4, 116-B-6A, 116-B-6B, $116-B-9,116-B-10,118-C-2,118-C-4,116-C-2 B, 116-C-2 C)$

3. $100 B / C$ Surface Contamination Areas (includes $116-C-5,116-B-11$, $116-B-13)$

4. 118-C-1 Burial Ground

5. 118-B-1 Burial Ground

6. $216-U-11$

7. $218-W-1,218-W-2,218-W-4 A, 218-W-4 B, 218-W-11$

8. 216-Z-4, 216-Z-5, 216-Z-6, 216-Z-10, 216-Z-17 Stabilized Areas

\section{Annua 1}

1. 200 East " $C$ " $C$ ribs and Trenches $(216-C-1,216-C-3,216-C-5,216-C-6$, $216-C-8,216-C-10)$

2. $216-5-16$

3. $216-S-19$

4. REDOX Railroad Spur

\section{Active Facilities}

\section{Weekly}

1. Routine water samples

\section{Biweekly}

1. Routine air samples

\section{Quarterly}

1. 244-A Lift Station Perimeter

\section{Semiannual}

1. 316-5 Process Trench - Perimeters

2. Route 4 South from 200 West Area Main Gate to 200 East Area Hill 
WHC-SP-0098-5

Rev. 0

Issue Date: $12 / 15 / 93$

FEBRUARY (cont.)

Active Facilities (cont.)

\section{Annual}

1. 216-B-3-3 Ditch surveys

2. West Lake survey plots $(216-\mathrm{N}-8)$

3. 216-T-4 Ditch and 216-T-4-2 pond

4. 307 Retention Basin Perimeter

5. $216-C-4$

6. $216-C-7$

7. 241-TX-155 Diversion BoX

8. 241-ER-152 Diversion Box

9. Original Central Landfill (located $1.5 \mathrm{mi}$ southeast of Army Loop Road, $100 \mathrm{ft}$ north of Highway 4 South)

10. Cross Site Transfer Line (200 East Area Section) UN-216-E-41

11. Route 4 South, 200 East Area Hill to Wye Barricade 
WHC-SP-0098-5

Rev. 0

Issue Date: $12 / 15 / 93$

\section{MARCH}

\section{Inactive Facilities}

\section{Quarterly}

1. Waste site visual survey (posting, housekeeping, vegetation control, animal activity, unusual conditions, etc.)

\section{Semiannual}

1. 100-D/DR Surface Contamination Areas (includes 116-D-7, 116-DR-9, and pipeline)

2. 216-B-3-1 and 216-B-3-2 Covered Ditches

\section{Annual}

1. 200 East Area " $B$ " Cribs and Trenches, Drains, and Reverse Wells (216-B-5, 216-B-6, 216-B-7A, 216-B-7B, 216-B-8, 216-B-9, 216-B-11A, 216-B-11B, $216-B-12,216-B-51,216-B-57$, and 216-B-43 through 216-B-50)

2. 100-D/DR URM Areas (includes 116-DR-1 and 116-DR-2 [107 trenches], $116-D R-5,116-D-5,116-D R-6,118-D-3$, and 118-D-4)

3. 105-D/DR Exclusion Areas (includes 116-D-1A, 116-D-1B, 116-D-2, 116-D-3, $116-D-4,116-D-6,116-D R-7,116-D R-3$, and 116-DR-4)

4. 118-D-1 Burial Ground

5. 118-D-2 Burial Ground

6. 118-DR-1 Burial Ground

7. 116-DR-8

\section{Active Facilities}

\section{Weekly}

1. Routine water samples

\section{Biweekly}

1. Routine air samples

\section{Bimonthly}

1. Survey of roads inside 200 East Area

2. Survey of roads inside 200 West Area 
MARCH (cont.)

\section{Active Facilities (cont.)}

\section{Quarterly}

1. TLD exchange 100 Area

2. TLD exchange $200 / 600$ Areas

3. TLD exchange $300 / 400$ Areas

4. 216-A-30 Crib

5. 216-B-55 Crib

6. 216-B-62 Crib

7. Waste site visual survey (posting, housekeeping, vegetation control, animal activity, unusual conditions, etc.)

\section{Semiannual}

1. 216-U-12 Crib

2. 216-U-16 Crib

3. 216-S-25 Crib

4. 216-A-8 Crib

5. 216-A-36A and 216-A-36B Cribs

6. 200/600 Areas Emergency Plot Surveys

7. 216-S-26 Crib

8. 216-U-17 $\mathrm{Crib}$

9. $216-A-37-1$

10. 216-A-37-2

\section{Annual}

1. $216-B-4$

2. $216-B-13$

3. 216-B-64

4. Cross Site Transfer Line from 200 East Area fence to access road near Vent Station (UN-216-E-41)

5. Unplanned release sites in 200 East Area: UN-216-E-7, UN-216-E-8, UN-216-E-10, UN-216-E-11, UN-216-E-13, UN-216-E-15, UN-216-E-18, UN-216-E-24, UN-216-E-26, and UN-216-E-38.

6. Highway survey, from entrance to 200 West Area to Route 11A, Route $11 \mathrm{~A}$ to Route 2 South, to Highway 240 via Route 10. 
WHC-SP-0098-5

Rev. 0

Issue Date: $12 / 15 / 93$

APRIL

Inactive Facilities

\section{Semiannual}

1. 216-B-2-1, 216-B-2-2, and 216-B-2-3 Covered Ditches

2. $218-E-8$

3. $218-E-12 A$

4. 218-E-12B (stabilized portion)

\section{Annua 1}

1. 100-F URM Area (includes 116-F-2, 116-F-5, 116-F-6, 116-F-8, 116-F-9, $116-\mathrm{F}-10,116-\mathrm{F}-12,116-\mathrm{F}-13$, and 118-F-3)

2. 105-F Exclusion Area (includes 116-F-3, 116-F-4, 116-F-11, and 118-F-4)

3. 107-F Retention Bas in (116-F-14)

4. 100-F Ash Disposal Area

5. 116-F-1 Lewis Canal

6. 118-F-1 Burial Ground

7. 118-F-2 Burial Ground

8. 118-F-5 Burial Ground

9. 118-F-6 Burial Ground

10. 618-1 Burial Ground

11. Cribs and trenches, drains, and reverse wells $(216-A-1,216-A-3,216-A-6$, 216-A-7, 216-A-9, and 216-A-27)

\section{Active Facilities}

\section{Weekly}

1. Routine water samples

\section{Biweekly}

1. Routine air samples

\section{Quarterly}

1. Tritium and nitrate water samples

2. Vent station perimeter and surface contamination area (cross site transfer line)

Semiannual

1. Railroad survey, 300 Area to 400 and 200 West Areas. To include all sidings and spurs external of fence boundaries of areas

2. 216-U-14 (active section from 207-U to 216-U-10 Pond) 
WHC-SP-0098-5

Rev. 0

Issue Date: 12/15/93

RPRIL (cont.)

Active Facilities (cont.)

\section{Annual}

1. UN-300-17 (333 Building)

2. UN-300-XX (333 Building)

3. $216-A-40$

4. 216-T-1 Ditch Perimeter

5. 300/400 Areas Soll and Vegetation Sampling

6. Cross Site Transfer Line from access road near Vent Station to 200 West Area fence (UN-216-E-44)

7. Highway survey, Yakima Barricade to Army Loop Road (Route 11A), Army Loop Road to Route 4 South 
WHC-SP-0098-5

Rev. 0

Issue Date: 12/15/93

MAY

Inactive Facilities

Semiannual

1. " $B / C$ " Crib Ground Plots

2. 218-E-2, 218-E-5A, 218-E-5B, and 218-E-9 Burial Grounds

3. 218-E-10 Burial Ground

4. "B/C" Cribs, 216-B-14 through 216-B-19

5. "B/C" Trenches, 216-B-20 through 216-B-34, 216-B-52, 216-B-53A and 216-B-53B, 216-B-54, and 216-B-58

6. " $B / C$ " Cribs Controlied Area Roads and Firebreaks

\section{Annual}

1. 100-H URM Area (includes 116- $\mathrm{H}-1,116-\mathrm{H}-2$, and 116- $\mathrm{H}-5$ )

2. 105-H Exclusion Area (includes $116-\mathrm{H}-3,118-\mathrm{H}-4$, and $118-\mathrm{H}-5$ )

3. 116-H-6 Solar Basin (183-H)

4. 116-H-7 Retention Basin

5. 118-H-1 Burial Ground

6. 118-H-2 Burial Ground

7. 118-H-3 Burial Ground

8. 316-1 South Process Pond

9. 316-2 North Process Pond

\section{Active Facilities}

\section{Weekly}

1. Routine water samples

\section{Biweekly}

1. Routine air samples

Bimonthly

1. Road survey inside 200 East Area

2. Road survey inside 200 West Area

\section{Quarterly}

1. 244-A Lift Station Perimeter 
WHC-SP-0098-5

Rev, 0

Issue Date: $12 / 15 / 93$

MAY (cont.)

Active Facilities (cont.)

Semiannual

1. 200 West Area Tank Farm Perimeters (241-T; 241-TX and 241-TY; 241-S, 241-SX, and 241-SY; and 241-U)

2. 200 East Area Tank Farm Perimeters (241-A, 241-AN, 241-AX, 241-AY, and 241-AZ; 241-AP; 241-AW; 241-B; 241-BX and 241-BY; and 241-C)

\section{Annual}

1. 200/600 Areas soil and vegetation sampling

$2200 / 600$ Areas pond and ditch aquatic vegetation sampling

3. 200/600 Areas pond and ditch sediment sampling

4. 400 Area Process Pond perimeters

5. Highway survey, 100-N Area to intersection of Route 11 A (Route 2 North) to include all parking areas, paved access roads to the 100 Areas and Route 1 from the Hanford town site to 100-B Area 
WHC-SP-0098-5

Rev. 0

Issue Date: $12 / 15 / 93$

\section{JUNE}

\section{Inactive Facilities}

\section{Quarterly}

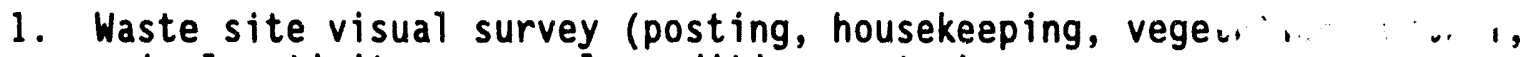
animal activity, unusual conditions, etc.)

\section{Annual}

1. 100-K URM Area (includes 116-KW-4, 116-KE-5, 116-K-3 outfall)

2. 105-K Exclusion Area (includes 116-KW-1, 116-KW-2, 116-KE-1, 116-KE-2, $116-K E-3,118-K W-2,118-K E-2)$

3. 107-KE/KW Retention Basins (116-KW-3, 116-KE-4)

4. 100-K Flood Plain Surface Contamination Area

5. 118-K-1 Burial Ground

6. 116-K-1 Crib

7. 116-K-2 (mile-long trench)

8. 216-U-14 Ditch (inactive section from 19th Street to 207-U Basin)

9. 216-T Cribs, Trenches, and Reverse Wells (216-T-2, 216-T-3, and 241-T-361

Catch Tank; 216-T-5 tile field [to include 216-T-7]; 216-T-6; ?.16-T-8;

216-T-18; 216-T-19; 216-T-20; 216-T-26 through 216-T-28; 216-T-33;

$216-T-34 ; 216-T-35 ;$ and $216-T-36$

10. 204-S Stabilized Area

11. 216-T-14 through 216-T-17

12. 216-U-10 Covered Pond (incl -216-W-14, UN-216-W-15, UN-216-W-16, and UN-216-W-17)

13. 218-W-1A Burial Ground

14. 218-W-2A Burial Grouno

15. 218-W-3 Burial Ground

16. 216-Z-19 and 216-Z-20 Covered Ditches (includes UN-216-W-20)

17. 216-T-21 through 216-T-25 Trenches

\section{Active Facilities}

\section{Weekly}

1. Routine water samples

\section{Biweekly}

1. Routine air samples 
WHC-SP-0098-5

Rev. 0

Issue Date: $12 / 15 / 93$

JUNE (cont.)

Active Facilities (cont.)

Quarterly

1. TLD exchange 100 Area

2. TLD exchange $200 / 600$ Areas

3. TLD exchange $300 / 400$ Areas

4. $216-\mathrm{A}-30 \mathrm{Crib}$

5. 216-B-62 Crib

6. 216-B-55 Crib

7. Waste site visual survey (posting, housekeeping, vegetation control, animal activity, unusual conditions, etc.)

\section{Annual}

1. RR Track near Willa Junction, to include UN-216-W-31

2. Highway survey, West Lake to Route 6 (100-B Area cutoff), Route 6 to Route IIA, Route $11 \mathrm{~A}$ to Intersection of Route 3

3. Rail survey, 200 West Area railways, spurs, and sidings 
WHC-SP-0098-5

Rev. 0

Issue Date: $12 / 15 / 93$

JULY

Inactive Facilities

\section{Semiannual}

1. UN-216-W-5

\section{Annual}

1. $U N-216-W-7$

2. 218-W-7 Burial Vault

3. 218-W-8 Burial Vault

4. 218-W-9 Burial Vault (includes UN-216-W-19)

5. 218-E-7 Burial Vault

6. 216-Z Cribs and Trenches (216-Z-7, 216-Z-8, 216-Z-12, 216-Z-16, 216-Z-18)

7. 207-S Retention Basin (includes UN-216-W-2)

8. $618-2$

9. $618-3$

10. $618-5$

11. $618-10$

12. $618-11$

\section{Active Facilities}

\section{Weekly}

1. Routine water samples

\section{Biweekly}

1. Routine air samples

\section{Bimonthly}

1. Road surveys inside 200 East Area

2. Road surveys inside 200 West Area

\section{Quarterly}

1. Tritium and nitrate water samples

2. Vent station perimeter and surface contamination area (cross site transfer line)

\section{Semiannual}

1. Composite air samples

2. Outside perimeter roads of 200 East Area

3. Outside perimeter roads of 200 West Area 
WHC-SP-0098-5

Rev. 0

Issue Date: $12 / 15 / 93$

JULY (cont.)

\section{Active Facilities (cont.)}

\section{Annual}

1. 216-Z-9

2. 207-B Retention Basin Perimeter

3. 207-SL Retention Basin Perimeter

4. 207-T Retention Bas in Perimeter (includes 216-T-12 Sludge Pit)

5. 207-U Retention Bas in Perimeter (includes UN-216-W-21 and UN-216-W-22)

6. 216-A-42 Retention Basin Perimeter (207-AA)

7. 100 Area soil and vegetation sampling

8. 100-N Area Micro R Survey

9. Highway survey, from intersection of Route 3 and Route 4 North to 100-N Area

10. Rail survey, 200 East Area railways, spurs, and sidings 
WHC-SP-0098-5

Rev. 0

Issue Date: $12 / 15 / 93$

\section{AUGUST}

\section{Inactive Facilities}

\section{Semi annual}

1. $100-B / C$ Areas (includes 116-B-1, 116-B-2, 116-B-5, 116-B-7, 116-B-8, $116-B-13,116-B-14,116-C-1,116-C-2 A, 116-C-4,118-B-2,118-B-3$, $118-B-4,118-B-5,118-B-6,118-B-7,116-C-5,116-B-11)$

2. $105-B / C$ Exclusion Areas (includes 116-B-3, 116-B-4, 116-B-6A, 116-B-6B, $116-B-9,116-B-10,118-C-2,118-C-4,116-C-2 B$, and $116-C-2 C)$

3. 118-C-1 Burial Ground

4. 118-B-1 Burial Ground

5. $216-U-11$

6. $218-W-1,218-W-2,218-W-4 A, 218-W-4 B$, and $218-W-11$

7. 216-Z-4, 216-Z-5, 216-Z-6, 216-Z-10, and 216-Z-17 Stabilized Areas

\section{Annual}

1. 316-3 Retired Trenches

2. 216-S Cribs and Trenches (216-S-1, 216-S-2, 216-S-4, 216-S-5, 216-S-6, 216-S-172, and 2904-S-160 Weirs; 216-S-7, 216-S-8, 216-S-9, 216-S-12, $216-S-13,216-S-20,216-S-21,216-S-22,216-S-23)$

3. 216-U Cribs and Trenches (216-U-1, 216-U-2, 216-U-3, 216-U-5, 216-U-6, 216-U-8)

\section{Active Facilities}

\section{Weekly}

1. Routine water samples

\section{Biweekly}

1. Routine air samples

Quarterly

1. 244-A Lift Station Perimeter

\section{Semiannual}

1. 316-5 Process Trench Perimeter

2. Route 4 South from 200 West Area Main Gate to 200 East Area Hill 
WHC-SP-0098-5

Rev. 0

Issue Date: $12 / 15 / 93$

\section{AUGUST (cont.)}

\section{Active Facilities (cont.)}

\section{Annua 1}

1. Underground pipeline from $216-\mathrm{B}-2-3$ to $216-\mathrm{B}-3-3$

2. UN-300-5 (309 Tank Farms)

3. UN-200-W-8 (01d Burial Ground east of U Plant)

4. 216-B-3 Pond Perimeter Survey

5. 216-B-63 Ditch Survey

6. 216-S-10 Ditch Active

7. Unplanned Release Site Surveys 200 West Area: UN-216-W-4, UN-216-W-6, UN-216-W-12, UN-216-W-13, and UN-216-W-28

8. Rail survey, 300 Area to Columbia Center to include all sidings and spurs 
WHC-SP-0098-5

Rev. 0

Issue Date: $12 / 15 / 93$

\section{SEPTEMBER}

\section{Inactive Facilities}

\section{Quarterly}

1. Waste site visual survey (posting, housekeeping, vegetation control, animal activity, unusual conditions, etc.)

\section{Semiannual}

1. 216-B-3-1 and 216-B-3-2 Covered Ditches

2. 100-D/DR Surface Contamination Areas (includes 116-D-7, 116-DR-9, and pipeline)

\section{Annual}

1. UN-216-E-17

2. 216-A-25 Covered Pond

3. D Island Vent risers and surrounding area

4. 216-A-10 Crib

5. 216-B-10A and 216-B-10B

6. UN-216-E-33

7. $216-A-24$

8. $216-A-34$

9. $216-A-18$

10. $216-A-19$ and $216-A-20$

11. 216-A-29 Ditch

Active Facilities

\section{Weekly}

1. Routine water samples

\section{Biweekly}

1. Routine air samples

\section{Bimonthly}

1. Survey of roads inside 200 East Area

2. Survey of roads inside 200 West Area 
WHC-SP-0098-;

Rev. 0

Issue Date: 12/15/93

SEPTEMBER (cont.)

Active Facilities (cont.)

\section{Quarterly}

1. TLD exchange, 100 Area

2. TLD exchange, $200 / 600$ Areas

3. TLD exchange, $300 / 400$ Areas

4. 216-A-30 Crib

5. 215-B-55 Crib

6. 216-B-62 Crib

7. Waste site visual survey (posting, housekeeping, vegetation control, animal activity, unusual conditions, etc.)

\section{Semiannual}

1. 216-A-36A and 216-A-36B Cribs

2. $216-\mathrm{S}-25 \mathrm{Crib}$

3. 216-U-16 Crib

4. 216-U-12 Crib

5. 200/600 Areas Emergency Plot Surveys

6. $216-\mathrm{U}-17 \mathrm{Crib}$

7. 216-S-26 Crib

8. 216-A-37-1 Crib

9. 216-A-37-2 Crib

10. 216-A-8 Crib

\section{Annual}

1. N Springs water sampling

2. Unplanned Release Site Survey 200 West Area: UN-216-E-5, UN-216-E-6, UN-216-E-12 perimeter, UN-216-E-14, UN-216-E-16, UN-216-E-23, $U N-216-E-30, U N-216-E-34, U N-216-E-36, U N-216-E-43$, and UN-216-E-44

3. 216-A-45 Crib 
WHC-SP-0098-5

Rev. 0

Issue Date: $12 / 15 / 93$

\section{OCTOBER}

\section{Inactive Facilities}

\section{Semiannual}

1. 218-E-12A

2. 218-E-12B Stabilized Portion (includes UN-216-E-32)

3. $218-E-8$

4. 216-B-2-1, 216-B-2-2, and 216-B-2-3

\section{Annual}

1. 216-N-1 through 216-N-7 (seven surveys)

2. Unplanned Release Site Surveys 200 West Area: UN-216-W-24, UN-216-W-25, UN-216-W-26, UN-216-W-29, UN-216-W-30, and UN-216-W-31

3. 216-B-2-1, 216-B-2-2, and 216-B-2-3 Covered Ditches

4. 216-B-35 through 216-B-42 Trenches

5. $218-E-1$

6. $218-E-2 A$

7. $218-E-4$

8. $218-C-9$

\section{Active Facilities}

\section{Weekly}

1. Routine water samples

\section{Biweekly}

1. Routine air samples

\section{Quarterly}

1. Tritium and nitrate water samples

2. Vent station perimeter and surface contamination area (cross site transfer line)

\section{Semiannual}

1. Railroad survey, 300 Area to 400 and 200 West Areas. To include all sidings and spurs external of fence boundaries of areas

2. $216-U-14$ (active section from $207-U$ to $216-U-10$ Pond) 


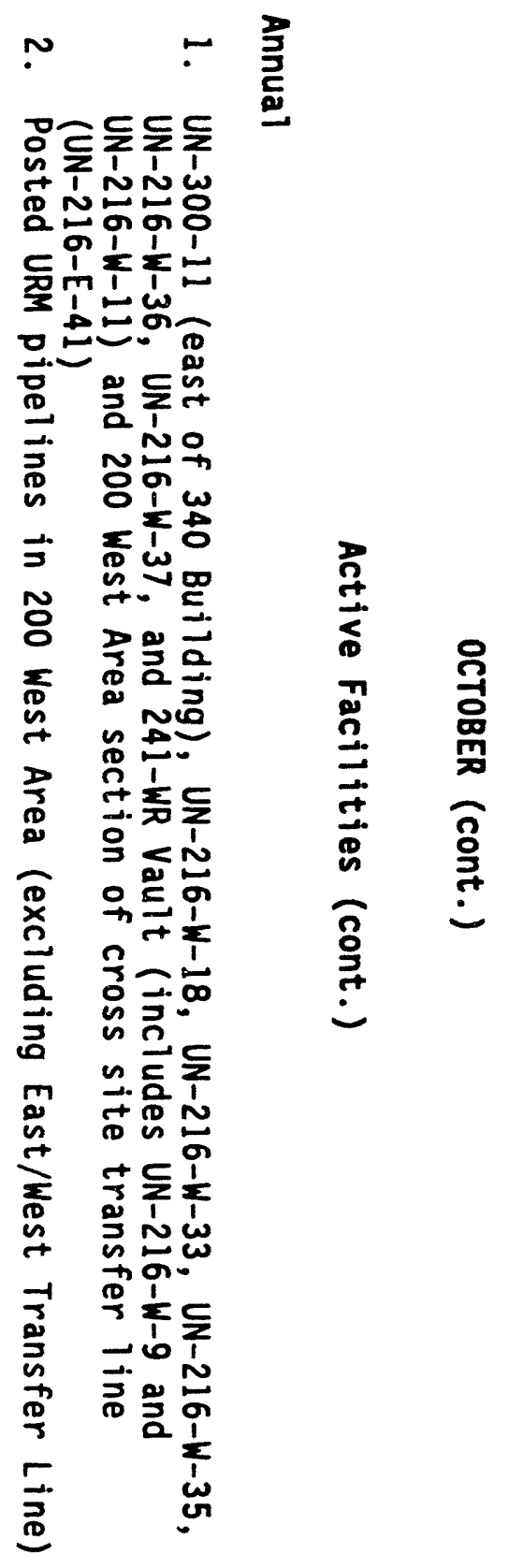




\section{NOVEMBER}

\section{Inactive Faciities}

\section{Semi annual}

1. "B/C" Crib Ground Plots

2. 218-E-2, 218-E-5A, 218-E-5B, and 218-E-9 Burial Grounds

3. 218-E-10 Burial Ground

4. $" B / C^{n}$ Cribs, 216-B-14 through 216-B-19

5. "B/C" Trenches, 216-B-20 through 216-B-34, 216-B-52, 216-B-53A and 216-B-53B, 216-B-54, 216-B-58

6. $" B / C$ " Cribs Controlled Area Roads and Firebreaks

\section{Annual}

1. 618-4 Burial Ground

2. 618-7 Burial Ground

3. 618-8 Burial Ground

\section{Active Facilities}

\section{Weekly}

1. Routine water samples

\section{Biweekly}

1. Routine air samples

\section{Bimonthly}

1. Road surveys inside 200 East Area

2. Road surveys inside 200 West Area

\section{Quarterly}

1. 244-A Lift Station Perimeter

\section{Semiannual}

1. 200 West Area Tank Farm Perimeters (241-T; 241-TX and 241-TY; 24I-S, 241-SX, and 241-SY; and 241-U)

2. 200 East Area Tank Farm Perimeters (241-A, 241-AN, 241-AX, 241-AY, and 241-AZ; 241-AP; 241-AW; 241-B; 241-BX and 241-BY; and 241-C) 
WHC-SP-0098-5

Rev. 0

Issue Date: $12 / 15 / 93$

NOVEMBER (cont.)

Active Facilities (cont.)

\section{Annual}

1. Outside perimeters of all active 200 West Area Burial Grounds (218-W-3A, 218-W-3AE, 218-W-4B, 218-W-4C, 218-W-5)

2. Outside perimeters of all active 200 East Area Burial Grounds (218-E-12B and 218-E-15) 
WHC-SP-0098-5

Rev. 0

Issue Date: $12 / 15 / 93$

\section{DECEMBER}

\section{Inactive Facilities}

\section{Quarterly}

1. Waste site visual survey (posting, housekeeping, vegetation control, animal activity, unusual conditions, etc.)

\section{Annual}

1. Special surveys (e.g., fresh animal feces, pipelines). Contact Environmental Engineering Studies

\section{Active Facilities}

\section{Weekly}

1. Routine water samples

\section{Biweekly}

1. Routine air samples

\section{Quarterly}

1. TLD exchange, 100 Area

2. TLD exchange, $200 / 600$ Areas

3. TLD exchange, 300/400 Areas

4. $216-\mathrm{A}-30 \mathrm{Crib}$

5. 215-B-55 Crib

6. 216-B-62 Crib

7. Waste site visual survey (posting, housekeeping, vegetation control, animal activity, unusual conditions, etc.)

\section{Annual}

1. UN-300-4 (321 Building)

2. UN-300-40 (303-F Pipe Trench)

3. Special surveys (e.g., fresh animal feces, pipelines). Contact Environmental Engineering Studies 
Number of copies

ONSITE

3

U.S. Department of Energy, Richland Operations office

R. F. Brich

A5-55

M. J. Furman

R3-80

J. B. Hall

A5-55

Pacific Northwest Laboratory

R. K. Woodruff (2)

K6-61

Westinghouse Hanford Company
H. A. Besel
L. P. Diediker
J. J. Dorian
D. S. Gunnink (6)
J. E. Hodgson
M. C. Hughes
A. R. Johnson
S. M. Mckinney
D. L. Mitchell
W. L. Osborne
C. J. Perkins (6)
J. W. Schmidt (10)
J. A. Seamans
K. A. Smith
R. R. Thompson
C. D. Wade (6)
D. J. Watson
R. L. Watts
M. A. Wendling
Central Files
Document Processing and Distribution (2)
EDMC
Information Release Administration
T1-27
T1-30
H6-30
T1-25
X7-02
R2-81
H6-30
T1-30
H6-32
T3-11
$\times 0-21$
H6-30
N2-04
$\mathrm{N} 1-06$
$\mathrm{H} 6-32$
$\mathrm{N1}-06$
X0-41
L6-52
$\mathrm{N} 1-06$
L8-04
L8-15
H6-08
H4-17 